\title{
Quantum networking with atomic ensembles
}

\author{
A Thesis \\ Presented to \\ The Academic Faculty \\ by

\section{Dzmitry Matsukevich}

In Partial Fulfillment

of the Requirements for the Degree

Doctor of Philosophy

School of Physics

Georgia Institute of Technology

August 2006 


\section{Quantum networking with atomic ensembles}

Approved by:

Alex Kuzmich, Advisor

School of Physics

Georgia Institute of Technology

Brian Kennedy

School of Physics

Georgia Institute of Technology

Michael Chapman

School of Physics

Georgia Institute of Technology
Chandra Raman

School of Physics

Georgia Institute of Technology

Paul Voss

School of Electrical

and Computer Engineering

Georgia Institute of Technology

Date Approved: March, 29, 2006 
Dedicated to the dog which I never had. 


\section{ACKNOWLEDGEMENTS}

First, I would like to thank Alex Kuzmich for his support, guidance and enthusiasm.

This thesis would not be possible without Thierry Chanelière. We did most of the work together and I have learned a lot from him. I am indebted to Stewart Jenkins and Brian Kennedy for guidance and advice on the theoretical aspects of this work.

I would like to thank Michael Chapman and all the members of his group for numerous discussions and for all the equipment that I have borrowed from their laboratory over the past three years.

I would like also to thank all the past and present members of our research group: ShauYu Lan, Ryan Smith, Tamar Neumann, Corey Campbell, Ran Zhao, Mishkat Bhattacharya, Stephen Rafter, Andrew Cheung, Austin Collins, thank you very much for your help.

Finally, I would like to thank my family for their support and encouragement. 


\section{TABLE OF CONTENTS}

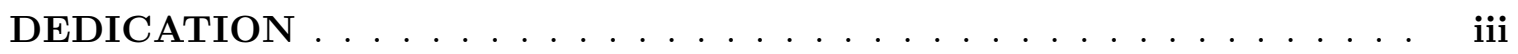

ACKNOWLEDGEMENTS .................... iv

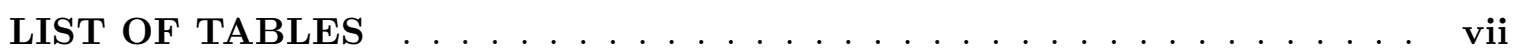

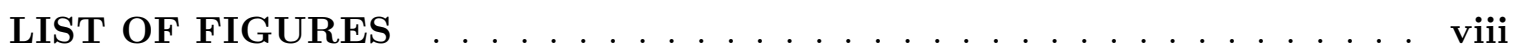

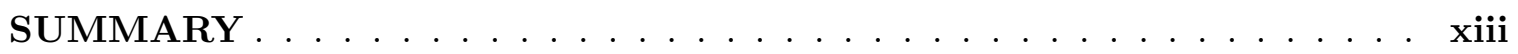

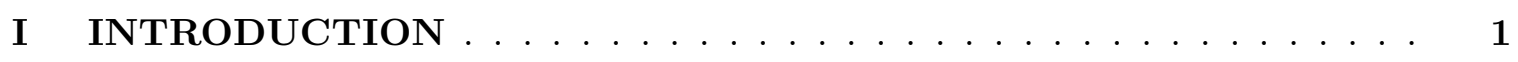

1.1 Quantum communication ..................... 1

1.2 Atomic ensembles . . . . . . . . . . . . . . . 2

1.3 Outline .............................. 4

1.4 Electromagnetically induced transparency and dark state polariton . . . . 6

1.5 DLCZ protocol for the generation of photon pairs . . . . . . . . 9

II STORAGE AND RETRIEVAL OF SINGLE PHOTONS TRANSMITTED BETWEEN REMOTE QUANTUM MEMORIES . . . . . . . . . 11

2.1 Motivation . . . . . . . . . . . . . . . . . . . 11

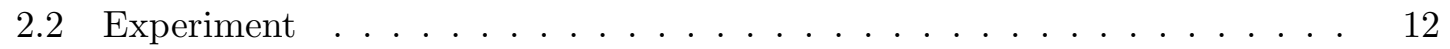

2.3 Measurement procedure . . . . . . . . . . . . . . 20

2.4 Photoelectric counting statistics . . . . . . . . . . . . . 21

III OBSERVATION OF DARK STATE POLARITON COLLAPSES AND

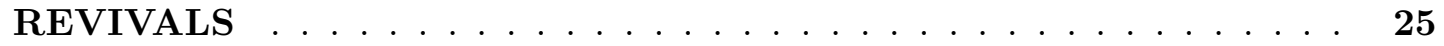

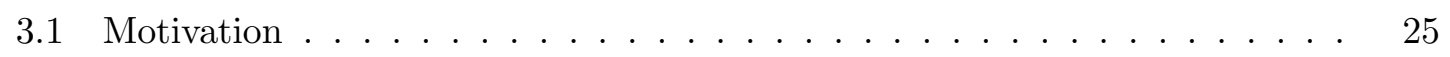

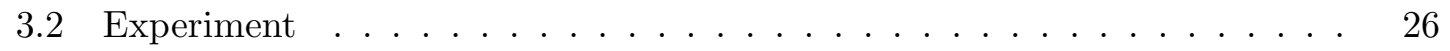

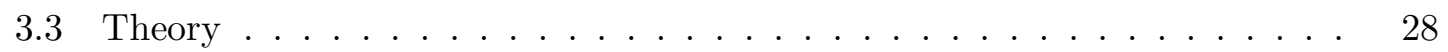

IV DETERMINISTIC SINGLE PHOTONS VIA CONDITIONAL QUANTUM EVOLUTION . . . . . . . . . . . . . . . . . . 35

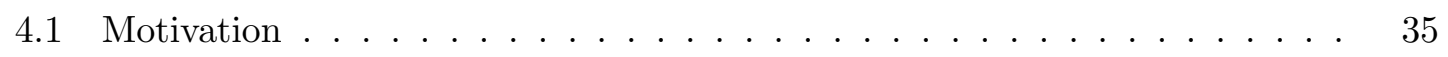

4.2 Protocol for deterministic single photon generation $\ldots \ldots \ldots$

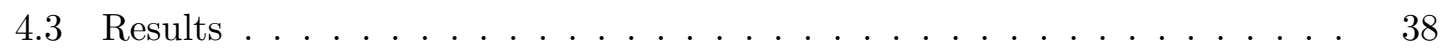


V QUANTUM STATE TRANSFER BETWEEN MATTER AND LIGHT 44

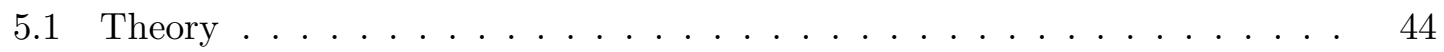

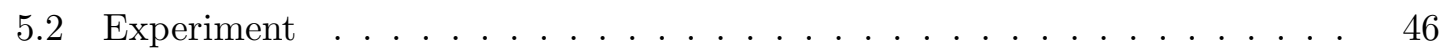

VI ENTANGLEMENT OF A PHOTON AND A COLLECTIVE ATOMIC

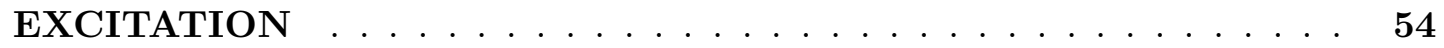

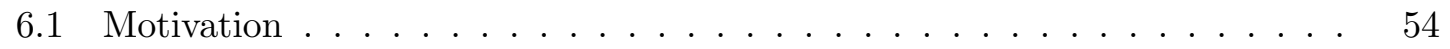

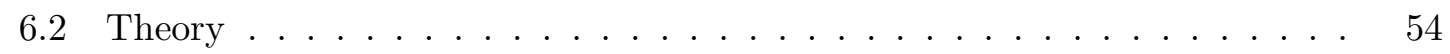

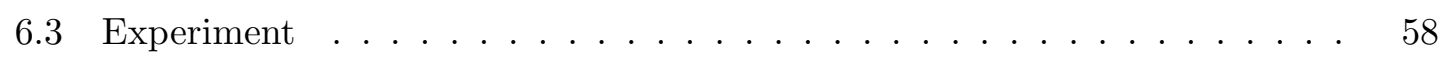

VII ENTANGLEMENT OF REMOTE ATOMIC QUBITS . . . . . . . . . . 61

7.1 Introduction . . . . . . . . . . . . . . . . . . . 61

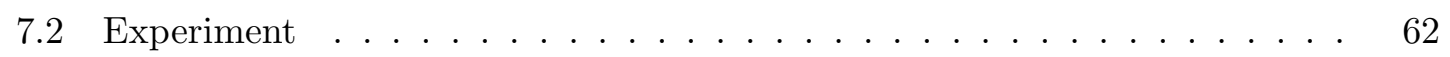

7.3 Bell inequality violation $\ldots \ldots \ldots \ldots \ldots$

7.4 Fidelity of entanglement $\ldots \ldots \ldots \ldots \ldots$

VIIRUANTUM TELECOMMUNICATION BASED ON ATOMIC CAS-

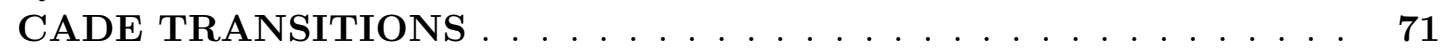

8.1 Motivation . . . . . . . . . . . . . . . 71

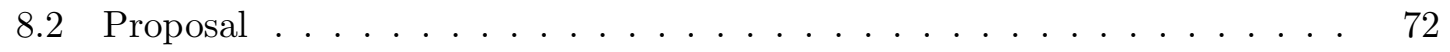

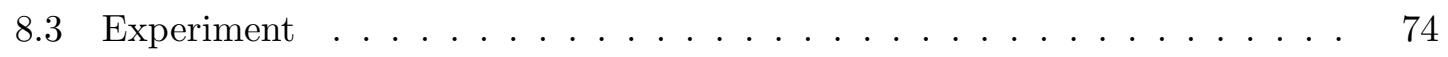

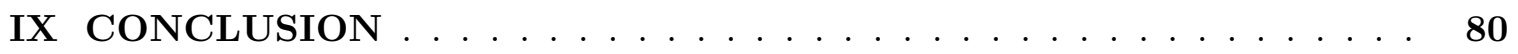

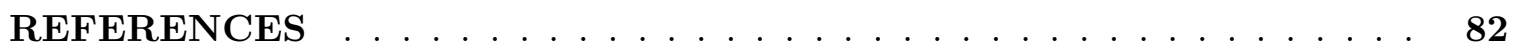




\section{LIST OF TABLES}

1 Conditional probabilities $P(I \mid S)$ to detect the idler photon in state $I$ given detection of the signal photon in state $S$, at the point of maximum correlation for $\Delta t=100 \mathrm{~ns}$ delay between read and write pulses; all the errors are statistical. . . . . . . . . . . . . . . . . .

2 Measured correlation function $E\left(\theta_{s}, \theta_{i}\right)$ and $S$ for $\Delta t=200$ ns delay between write and read pulses; all the errors are based on the statistics of the photon counting events. . . . . . . . . . . . . . .

3 Measured values of the correlation function $E\left(\theta_{A}, \theta_{B}\right)$ at particular polarization settings and the Bell parameter $S \ldots \ldots \ldots \ldots$

$4 \quad$ Inferred density matrix elements $\rho_{i j}$. Error bars represent \pm one standard deviation and are based on the statistics of the photoelectric counting events. 70

$5 \quad$ Measured correlation function $E\left(\theta_{s}, \theta_{i}\right)$ and $S$ for $\lambda_{s}=776 \mathrm{~nm}$ and $\lambda_{s}=1.53$

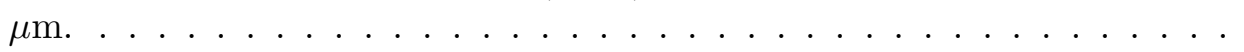




\section{LIST OF FIGURES}

1 Scheme of atomic levels illustrating EIT process. Strong coupling field is on resonance with $e \rightarrow g^{\prime}$ transition, and a weak signal field is resonant with the $e \rightarrow g^{\prime}$ transition. . . . . . . . . . . . . .

2 Real (top) and imaginary (bottom) parts of refraction index (a. u.) as a function of light detuning. We assume that $\gamma_{g g^{\prime}}=0$ and $\Omega / \gamma_{g e}=1$. . . .

3 DLCZ protocol for the photon pair generation. Part A illustrates write process, Part B illustrates read process. . . . . . . . . . . . . .

4 A schematic diagram of our experimental setup demonstrating generation, transmission, storage and retrieval of single photon excitations of the electromagnetic field. Two atomic ensembles at Sites $A$ and $B$ are connected by a single-mode fiber. The insets show the structure and the initial populations of atomic levels for the two ensembles. All the light fields responsible for trapping and cooling, as well as the quadrupole magnetic fields in both MOTs, are shut off during the period of the protocol. The ambient magnetic field at each Site is compensated by three pairs of Helmholtz coils (not shown). Correlated signal and idler fields are generated at Site $A$. The signal field is transmitted via optical fiber from Site $A$ to Site $B$, where it is converted to atomic excitation, stored for a duration $T_{s}$, and subsequently retrieved. A Hanbury Brown-Twiss setup consisting of a beamsplitter BS and two detectors D2 and D3, together with detector D1 for the idler field, are used to verify the single photon character of the retrieved field. . . . .

5 Measured transmission spectra of a coherent probe field as the function of probe detuning in the presence of, and absence of, EIT. Data are taken using $700 \mathrm{~ns}$ long coherent laser pulses. $T$ is the intensity transmittance, $\Delta$ is the probe detuning and $\Gamma$ is the decay rate of level $|c\rangle$. In the absence of control field (circles) the probe is strongly absorbed near resonance, whereas with the control field on (diamonds) the medium becomes transparent. Each probe pulse contains on average 0.3 photons. Each data point is an average of $2 \times 10^{5}$ experimental trials. The optical thickness $d=8$ and the control field Rabi frequency $\Omega=3 \Gamma$ are used to obtain the solid curves, based on the theoretical model discussed in this chapter. . . . . . . . . . . . .

6 Experimental and theoretical pulse shapes as a function of time, showing EIT, storage and retrieval. The color code is: control field - black, pulse in vacuum - blue, delayed, stored and retrieved field - red. Panel (a) with a cw control field shows EIT pulse delay. In panel (b) the control field is switched off and then on again after $500 \mathrm{~ns}$, shows light storage and retrieval. Panel (c) is similar to (b) but with a $15 \mu$ s storage. Panels (d), (e), and (f) are corresponding theoretical plots. . . . . . . . . . . . . . 
Measured intensity cross-correlation function $g_{s i}$ and anticorrelation function $\alpha$ as a function of the idler photoelectric detection probability $p_{1}$. Panels (a) and (b) are for the source (propagation in vacuum). Panels (c) and (d) are for stopped, stored for $500 \mathrm{~ns}$, and retrieved signal field. The solid lines are based on a theoretical model that includes losses and background. Error bars represent \pm one standard deviation and are based on the statistics of the photoelectric counting events. . . . . . . . . . . .

8 Normalized signal-idler intensity correlation function $g_{s i}$ as a function of the storage time $T_{s}$ at Site $B$. Data (diamonds) are taken for $p_{1}=0.0047$, but with a smaller background contribution than that of Fig.4, $\mathrm{c}$ and d. The full curve is a fit of the form $1+B \exp \left(-t^{2} / \tau^{2}\right)$ with $B=7$ and the collapse time $\tau=11 \mu \mathrm{s}$ as adjustable parameters. Error bars represent \pm one standard deviation and are based on the statistics of the photoelectric counting events. 19

$9 \quad$ Normalized intensity autocorrelation functions $g_{i i}$ (triangles) and $g_{s s}$ (circles for the source, squares for the stored and retrieved field). Uncertainties are based on the statistics of the photon counting events. . . . . . . . . .

10 Clauser parameter $R=g_{s i}^{2} /\left[g_{s s} g_{i i}\right]$ as a function of photodetection probability $p_{1}$ for the single photon source. . . . . . . . . . . . . . . . .

11 Clauser parameter $R=g_{s i}^{2} /\left[g_{s s} g_{i i}\right]$ as a function of photodetection probability $p_{1}$ after 500 ns storage time. . . . . . . . . . . . . .

12 A schematic diagram illustrates our experimental setup. A signal field from either a laser, or a DLCZ source of conditional single photons at Site $A$ is carried by a single-mode fiber to an atomic ensemble at Site $B$, where it is resonant on the $|b\rangle \leftrightarrow|c\rangle$ transition. The signal field is stored, for a duration $T_{s}$, and subsequently retrieved by time-dependent variation of a control field resonant between levels $|a\rangle$ and $|c\rangle$. All the light fields responsible for trapping and cooling, as well as the quadrupole magnetic field in the MOT, are shut off during the period of the storage and retrieval process. An externally applied magnetic field created by three pairs of Helmholtz coils (not shown) makes an angle $\theta$ with the signal field wavevector. The inset shows the structure and the initial populations of atomic levels involved. The signal field is measured by detectors D2 and D3, while detector D1 is used in the conditional preparation of single photon states of the signal field at Site A. . . . . . . . . . . . . . . . . . . 
13 Panels (a)-(e) show the ratio of the number of photoelectric detection events for the retrieved and incident signal fields for various orientations, $\theta=$ $0, \pi / 8, \pi / 4,3 \pi / 8, \pi / 2$, of the applied magnetic field, and as a function of storage time. The incident signal field is a weak coherent laser pulse. In all cases the control pulse is switched off at $T_{s}=0$. We observe a series of collapses and revivals at multiples of the half Larmor period of $2.3 \mu \mathrm{s}$. The observed damping over several Larmor periods is likely caused by residual magnetic field gradients. The inset in Panel (e) demonstrates the observed substructure within the first Larmor period. Panels (f) through (j) are corresponding theoretical plots of the dark-state polariton number calculated using Eq.(14). . . . . . . . . . . . . . . . . . . . . . .

14 Diamonds show the measured collapse time $T_{C}$ of the first revival at half the Larmor period as a function of the measured revival time $T_{R}$, for magnetic field values of $0.8,0.6,0.4$, and $0.2 \mathrm{G}$, respectively, and for fixed orientation $\theta=\pi / 2$. The line shows the corresponding theoretical prediction $T_{C} \approx$ $0.082 T_{R}$ from Eq.(14) . . . . . . . . . . . . . . . . .

15 Squares show the measured rate of coincidence detections between D1 and D2,3, $N_{s i}=N_{12}+N_{13}$ as a function of the storage time. Diamonds show the measured level of random coincidences $N_{R}$. The ratio of squares to diamonds gives $g_{s i}$. Uncertainties are based on the statistics of the photoelectric counting events. . . . . . . . . . . . . . .

16 Schematic of experimental setup for deterministic single photon generation, with the inset showing the three-level atomic configuration involved (see text for details) . . . . . . . . . . . . . . . . . . .

17 Correlation functions $g_{s i}$ (panel (a)) and $\alpha$ (panel (b)) as a function of $p_{1}$, taken at $\tau=80 \mathrm{~ns}$. The solid lines are based on Eqs.(18,19), with addition of a nearly-negligible background contribution. . . . . . . . . . . .

18 Normalized signal-idler intensity correlation function $g_{s i}$ as a function of the storage time $\tau$. The full curve is a fit of the form $1+B \exp \left(-\tau^{2} / \tau_{c}^{2}\right)$ with $B=16$ and the collapse time $\tau_{c}=31.5 \mu$ s as adjustable parameters. . . . .

$19 g_{D}^{(2)}(0)$ as a function of maximum number of trials $N$ (panel (a)) and $p_{1}$ (panel (c)); measured efficiency to generate and detect a single photon $\eta_{D}$ as a function of $N$ (panel (b)) and $p_{1}$ (panel (d)). For panels (a) and (b) $p_{1}=0.003$, whereas for for panels (c) and (d) $N=150$. The full curves are based on Eqs. (21) and (22) with the values of efficiencies and coherence times given in the text, with however $\eta_{D}$ multiplied by an empirical factor of $2 / 3$. We believe this reduced efficiency is due to imperfect switching of the read light in the feedback-based protocol (we note that there are no other adjustable parameters in the simple theory presented). Evident deviations from the theory in panels (c) and (d), beyond the statistical uncertainties associated with photoelectric counting events, could be explained either by inadequacies of the theory, or slow systematic drifts in the residual magnetic field and the read light leakage. . . . . . . . . . . . . . . . . 
20 (A) Schematic of experimental setup. PBS1-6, polarizing beam splitters, $\lambda / 2$, half waveplate, polarization state transformers, $R_{s}\left(\theta_{s}, \phi_{s}\right)$ and $R_{i}\left(\theta_{i}, \phi_{i}\right)$, (D1,D2,D3), single photon detectors, DM, dichroic mirror. The inset illustrates the timing of the write and read pulses. (B) The relevant atomic level structure . . . . . . . . . . . . . . . . . . .

21 (A) Measured conditional probabilities $P\left(H_{i} \mid H_{s}\right)$ and $P\left(V_{i} \mid H_{s}\right)$ as the function of the polarization rotation $\theta_{s}$ of the signal photon. The full curves are fits with the visibility as the only adjustable parameter. (B) Measured conditional probabilities at the points of highest correlation. . . . . . . . .

22 (A) Measured conditional probabilities after $\theta_{i}=\pi / 4$ polarization rotation of the idler photon as the function of $\theta_{s}$. (B) Measured conditional probabilities at the points of highest correlation. . . . . . . . . . . .

23 Time-dependent entanglement fidelity of the signal and the idler $F_{s i}$; circles for $\Delta t=100 \mathrm{~ns}$, diamonds for $\Delta t=200 \mathrm{~ns} \ldots \ldots \ldots \ldots$

24 (a) Schematic of experimental setup. $P_{1}$ and $P_{2}$, polarizers; D1 and D2, detectors; $\lambda / 4$, quarter-waveplate. (b) The structure of atomic transitions leading to generation of atom-photon entanglement and of the subsequent read-out of atomic qubit. . . . . . . . . . . . . . . . . . .

25 Measured coincidence fringe for $\theta_{i}=67.5^{\circ}$. The curve is a fit based on Eq.(5), augmented by a background contribution, with $\eta=0.81 \times \pi / 4$, with visibility and amplitude being adjustable parameters. The visibility of the fit is $90 \%$. Uncertainties are based on the statistics of the photon counting

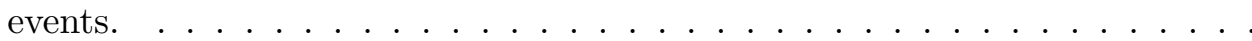

26 Normalized signal-idler intensity correlation function $g_{s i}$ as a function of storage time. Uncertainties are based on the statistics of the photon counting events. The full curve is the best exponential fit with time constant $\tau=3.7 \mu \mathrm{s}$. 60

27 A schematic diagram of our experimental setup. Two cold atomic ensembles of ${ }^{85} \mathrm{Rb}$, an unpolarized sample at Site $A$, and a spin-polarized sample at Site $B$, separated by $5.5 \mathrm{~m}$, are connected by a single-mode fiber. The insets show the structure and the initial populations of the atomic levels for the two ensembles. An entangled state of a collective atomic qubit and a signal field is generated at Site $A$ by Raman scattering of the write laser field. The orthogonal helicity states of the generated signal field are transmitted via optical fiber from Site $A$ to Site $B$, where they are converted to orthogonal collective atomic excitations, stored for a duration $T_{s}$, and subsequently converted into an idler field by adiabatic variation of the control field amplitude. The atomic qubit at Site $A$ is similarly converted into an idler field by a read laser pulse, counterpropagating with respect to the write pulse. For polarization analysis, each idler field propagates through a quarter-wave plate (not shown), a half-wave plate $(\lambda / 2)$ and a polarizing beamsplitter (PBS). Polarization correlations of the idler fields are recorded by photoelectric detection using the single photon detectors D1-D4. . . . . 
28 Measured coincidence fringes $C_{n 3}\left(\theta_{A}, \theta_{B}\right)$ as a function of $\theta_{A}$, for $\theta_{B}=135^{\circ}$, $n=1$, diamonds, $n=2$, squares. The curves are sinusoidal fits to the data. Each point is acquired for 15 minutes. The effective repetition rate is 108 $\mathrm{kHz}$, each trial takes $1.1 \mu \mathrm{s} . \ldots \ldots \ldots$

29 Measured correlation function $E\left(\theta_{A}, \theta_{B}\right)$ as a function of $\theta_{A}$. (a), $\theta_{B}=0^{\circ}$, squares, and $90^{\circ}$, diamonds. (b), $\theta_{B}=45^{\circ}$, squares, and $135^{\circ}$, diamonds. The curves are sinusoidal fits to the data. . . . . . . . . . . . . . . .

30 (a) The atomic structure for the proposed cascade emission scheme involving excitation by pumps I and II. Pump II and the signal photons lie in the telecommunication wavelength range when a suitable level of orbital angular momentum $L=0$ or $L=2$ is used as level $|d\rangle$. For atomic rubidium, the signal wavelength is $1.32 \mu \mathrm{m}\left(6 s_{1 / 2} \rightarrow 5 p_{1 / 2}\right.$ transition $), 1.37 \mu \mathrm{m}$ $\left(6 s_{1 / 2} \rightarrow 5 p_{3 / 2}\right.$ transition), $1.48 \mu \mathrm{m}\left(4 d_{3 / 2(5 / 2)} \rightarrow 5 p_{1 / 2}\right.$ transition $), 1.53 \mu \mathrm{m}$ $\left(4 d_{3 / 2(5 / 2)} \rightarrow 5 p_{3 / 2}\right.$ transition). For atomic cesium, the signal wavelength is $1.36 \mu \mathrm{m}\left(7 s_{1 / 2} \rightarrow 6 p_{1 / 2}\right.$ transition $), 1.47 \mu \mathrm{m}\left(7 s_{1 / 2} \rightarrow 6 p_{3 / 2}\right.$ transition). For $\mathrm{Na}$ and $\mathrm{K}$ the corresponding wavelengths are in the 1.1-1.4 $\mu \mathrm{m}$ range. (b) Schematic of experimental setup based on ultra-cold ${ }^{85} \mathrm{Rb}$ atomic gas. For $\lambda_{s}=776 \mathrm{~nm}$, phase matching results in the angles $\varepsilon^{\prime} \approx \varepsilon \approx 1^{\circ}$, while for $\lambda_{s}=1.53 \mu \mathrm{m}, \varepsilon^{\prime} \approx 2 \varepsilon \approx 2^{\circ}$. $P_{1}$ and $P_{2}$ are polarizers; D1 and D2 are

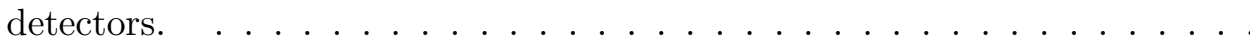

31 (a) Count rate proportional to the signal-idler intensity correlation function $G_{s i}$ as a function of signal-idler delay $\tau,|d\rangle=\left|5 d_{5 / 2}, F=4\right\rangle$. The quantum beats are associated with $120 \mathrm{MHz}$ hyperfine splitting, $F=3$ and 4 , of the $5 p_{3 / 2}$ level. The solid curve is a fit of the form $\beta+A \exp (-t / \alpha) \sin ^{2}(\pi \Omega t)$, where $\beta=63, A=2972, \alpha=11$ ns and $\Omega=117 \mathrm{MHz}$ are adjustable parameters. (b) Same as (a), but for $|d\rangle=\left|5 d_{5 / 2}, F=5\right\rangle$. Since this state can only decay though the $F=4$ component of the $5 p_{3 / 2}$ level, there are no quantum beats. The solid curve is an exponential fit with decay time of 3.2 ns. (c) The measured decay time vs the inverse measured optical thickness. (d) Measured coincidence fringes for $\theta_{s}=45^{\circ}$ (red diamonds) and $\theta_{s}=135^{\circ}$ (blue circles). The solid curves are fits based on Eqs. $(45,46)$, with $\cos \chi=1 / \sqrt{5} \ldots \ldots \ldots \ldots \ldots \ldots$

32 (a) Same as Fig. 31(a,b), but for $|d\rangle=\left|4 d_{5 / 2}, F=5\right\rangle$. The solid curve is an exponential fit with decay time of 6.7 ns. (b) Measured coincidence fringes for $\theta_{i}=45^{\circ}$ (red diamonds) and $\theta_{i}=135^{\circ}$ (blue circles). The solid curves are fits based on Eqs. $(45,46)$, with $\cos \chi=1 / \sqrt{5} \ldots \ldots \ldots$. . . . . . .

33 Efficiency of storage and subsequent retrieval of a coherent idler field with decay time of $6 \mathrm{~ns}$ in an auxiliary atomic ensemble, obtained by numerical integration of the Maxwell-Bloch equations. The atomic coherence time is assumed to be much longer than the storage time. . . . . . . . . . . . 78

34 Coherence time as a function of experiment date . . . . . . . . . . 81 


\section{SUMMARY}

Quantum communication networks enable secure transmission of information between remote sites. However, at present, photon losses in the optical fiber limit communication distances to less than 150 kilometers. The quantum repeater idea allows extension of these distances. In practice, it involves the ability to store quantum information for a long time in atomic systems and coherently transfer quantum states between matter and light. Previously known schemes involved atomic Raman transitions in the UV or nearinfrared and suffered from severe loss in optical fiber that precluded long-distance quantum communication.

In this thesis a practical quantum telecommunication scheme based on cascade atomic transitions is proposed, with particular reference to cold alkali metal ensembles. Within this proposal, essential building blocks for a quantum network architecture are demonstrated experimentally, including storage and retrieval of single photons transmitted between remote quantum memories, collapses and revivals of quantum memories, deterministic generation of single photons via conditional quantum evolution, quantum state transfer between atomic and photonic qubits, entanglement of atomic and photonic qubits, entanglement of remote atomic qubits, and entanglement of a pair of $1530 \mathrm{~nm}$ and $780 \mathrm{~nm}$ photons. These results pave the way for construction of a realistic quantum repeater for long distance quantum communication. 


\section{CHAPTER I}

\section{INTRODUCTION}

\subsection{Quantum communication}

Quantum cryptography offers a secure way to transmit information between remote sites. It involves ability to entangle two distant qubits (two level quantum systems) [1,2]. These two qubits are used for quantum communication, either with the Ekert protocol that directly uses the entangled pair of qubits or the BB84 protocol that performs either remote state preparation or teleportation of a qubit $[3,4,5,6,7,8]$.

Parametric down conversion is an established technology to produce entangled photon pairs. For example it allows one to teleport the quantum state of a photon and to achieve secure quantum key distribution over distances of more than hundred kilometers [6,9]. In order to further extend communication length, Zeilinger and coworkers proposed using a satellite for distribution of entangled photon pairs [10]. Unfortunately it is difficult to extend the approach of parametric down conversion over distances much longer than the photon absorption length. Because of the inevitable signal losses in the optical fibers and the probabilistic nature of the photon pair generation, the communication rate decreases exponentially with distance.

The concept of a quantum repeater was proposed [2] to overcome this limitation and enable quantum communications over longer distance. The idea is to insert quantum memory elements into the quantum channel every attenuation length or so. Then entanglement between neighbouring pairs of qubits is generated efficiently, since light is not appreciably absorbed within the segment length. After entanglement between pairs of atomic qubits is established, a joint measurement on neighboring pair of qubits is performed. Quantum states of the intermediate qubits are destroyed by the measurement, thus achieving entan-

glement swapping so that entanglement is extended to twice the initial distance. Subsequent 
purification and swapping steps using a nested purification protocol eventually result in entanglement of two particles at distant locations. The communication rate in this case scales polynomially with the distance $[2,11]$.

The quantum repeater is one of the possible examples of a quantum network. In general, a quantum network consists of spatially separated nodes to store and process quantum information and quantum channels that connect the nodes. Atoms are excellent candidates for storage and manipulation of quantum states, because it is possible to isolate atoms from the environment and manipulate their internal states with laser light or external DC fields. Photons are ideal carriers of quantum information, because they can propagate over long distances in free space or in optical fibers.

There are several proposals for quantum network architectures, employing quantum repeaters, based on ensembles of atoms [11], single trapped ions [12], single atoms/ions strongly coupled to high-finesse optical cavities [13], and single solid state-based atomic emitters [14]. All of these proposals rely on atomic Raman transitions or assorted variations.

Important progress toward quantum networks was achieved recently in single atom and single ion experiments. In the microwave domain, single Rydberg atoms and single photons have been entangled [15]. An entangled state of an ion and a photon [16, 17] and neutral atom and photon $[18,19]$ recently has been reported. Cavity QED holds promise for generation of neutral atom-photon entanglement with a deterministic single photon source being an important step in that direction [20, 21].

\subsection{Atomic ensembles}

Collective enhancement of atom-photon interactions in optically thick atomic ensembles offers a somewhat simpler route towards quantum networks $[22,23,24,25,26,27,28]$. There is a long history in quantum optics of using atomic ensembles for studies of cooperative effects such as superradiance [29] and optical bistability (see, e.g., Ref. [30] and references therein). Recently the utility of optically thick atomic ensembles has been explored for multiparticle entanglement, generation of non-classical states of matter (e.g., squeezed states) [31, 32, 33], and continuous variable quantum information processing [31, 34, 35, 36, 37, 38, 23, 39]. 
An important breakthrough in the field of atomic ensembles is the discovery of electromagnetically induced transparency (EIT). An opaque atomic medium becomes transparent to the probe light if another (control) beam is applied [40,22]. EIT has also been used to demonstrate an ultraslow group velocity of light and to store and retrieve coherent light pulses in the atomic ensemble [41, 42, 26, 27].

Duan, Lukin, Cirac, and Zoller (DLCZ) invented a protocol that realizes the quantum repeater architecture using atomic ensembles [11]. The protocol is a probabilistic scheme based upon entanglement of atomic ensembles via detection of single photon events in which the sources are intrinsically indistinguishable. The protocol generates entanglement over long distances via the quantum repeater architecture [2]. Inspired by DLCZ [11], emission of non-classical radiation has been observed in first-generation atomic ensemble experiments $[43,44,45]$.

Quantum state transfer between photonic- and matter-based quantum systems is a key element of quantum communication networks. The importance of quantum state transfer is rooted in the ability of atomic systems to provide excellent long-term quantum information storage, whereas the long-distance transmission of quantum information is currently accomplished using light.

In 2004 the first realization of coherent quantum state transfer from a matter qubit onto a photonic qubit was achieved [46]. This breakthrough laid the groundwork for several further advances toward the realization of a long-distance, distributed network of atomic qubits, linear optical elements, and single-photon detectors $[47,48,49,50]$. Important work toward the experimental realization of DLCZ scheme also is done by several other research groups. Non-classical correlations between fields from a single atomic ensemble have been observed in the single-photon regime in a hot vapor cell by Lukin's group at Harvard [51, 52].

Harris and coworkers pioneered off-axis four-wave mixing [53] and efficient photon-pair production [54] in a cold atomic ensemble using counter-propagating write and read fields deep in the regime of electromagnetically-induced transparency. The Vuletic group at MIT reported promising results for the photon pairs generation in an atomic ensemble placed in an optical cavity [55]. Kozuma and coworkers have recently demonstrated entanglement 
between Laguerre-Gaussian modes of a photon and the corresponding spatial modes of a single atomic ensemble [56].

\subsection{Outline}

All of the quantum repeater approaches described above rely on atomic Raman transitions or their variations. Such transitions in the ultraviolet to the near-infrared range have been successfully employed for entanglement generation [16, 46, 47, 48, 49, 18] making compact (a few kilometers) entanglement distribution conceivable. However in order to dramatically extend the reach of all these schemes, it is essential to be able to implement them at telecommunication wavelengths $(1.3 \mu \mathrm{m}-1.5 \mu \mathrm{m})$. Unfortunately, identifying suitable atomic Raman transitions at these wavelengths remains a challenge.

One possible approach to the long-distance quantum repeater could begin with generation of narrow-band, entangled photon pairs at telecommunication wavelength using intra-cavity parametric down conversion [13]. Clearly, these photon pairs satisfy the need to distribute the entanglement over long distances, but it is essential to convert at least one of the entangled photons into an atomic qubit. Coherent quantum state transfer between the telecommunication wavelength photon and atomic memory would require frequencyupconversion and subsequent mapping of the photon state onto an atomic qubit [13]. Frequency-upconversion of single telecommunication wavelength photons has been reported (e.g., $[57,58]$ and references therein) but not under conditions of wavelength and bandwidth suitable for storage in an atomic memory.

In this thesis we describe an alternative approach to the long distance quantum communication based on cascade transitions in atomic ensembles. In the alkali atoms the cascade atomic transition can be chosen so that the photon on the upper arm has the telecommunication wavelength while the photon on the lower arm is on resonance with the atomic transition to the ground state and is naturally suited for mapping to an atomic memory. Chapter 7 of this thesis reports experimental observation of entangled pairs of $1.5 \mu \mathrm{m}$ and $780 \mathrm{~nm}$ photons using the cascade scheme and describes our proposal for the long distance quantum repeater. 
The proposed quantum network architecture requires an ability to map the state of a photon to the state of an atomic ensemble. In Chapters 5 and 6 we discuss two different ways to store a qubit in an atomic ensemble and to entangle a photon and a collective atomic excitation. Chapter 5 describes observation of entanglement of an atomic qubit based on two independent atomic ensembles and a single photon and coherent quantum state transfer from atomic qubit to a single photon. In the experiment described in Chapter 6 an atomic qubit is encoded into two orthogonal spin excitations in the same atomic ensemble.

For the proposed architecture it is important to have a reversible coherent quantum state transfer between a photon and an atomic ensemble. Chapter 2 of this thesis is devoted to the generation of a single photon state in one laboratory and the subsequent storage and retrieval of this single photon state using an atomic ensemble in another laboratory utilizing the EIT effect. We show that the single photon character of the field is preserved in the storage and retrieval process. Chapter 7 describes the preparation of entangled states of two atomic ensembles. An entangled state of an atomic ensemble and a photon is generated in one laboratory. The photon is transmitted to another laboratory over an optical fiber, and is converted into an atomic qubit in another ensemble using the EIT approach. Entanglement of the two remote atomic qubits is inferred by performing, locally, quantum state transfer of each of the atomic qubits onto a photonic qubit and subsequent measurement of polarization correlations in violation of Bell inequality.

Another important ingredient for quantum networks is quantum feedback, i.e the ability to perform certain actions based on the outcome of the measurement. In Chapter 4 we propose a deterministic single photon source based on an ensemble of atomic emitters, measurement, and quantum feedback. We report the implementation of this scheme using a cold rubidium vapor. This source is stationary and produces a photoelectric detection record with truly sub-Poissonian statistics.

Manipulation of the quantum states of atomic quantum memory is another important goal for the quantum network. In Chapter 3 we describe collapses and revivals of quantum memory in a uniform magnetic field and study decoherence mechanisms in atomic ensembles. 
We have therefore demonstrated the essential building blocks of a long distance quantum repeater. In the next two sections some background material related to the quantum networks with atomic ensembles is discussed.

\subsection{Electromagnetically induced transparency and dark state polariton}

The absorption of light in an atomic medium is a function of the light frequency. As the frequency approaches an atomic resonance, the atom-field interaction strength increases making the medium opaque to the incident light. EIT renders the medium transparent by using the quantum interference effect due to a strong control laser field.

Let us assume that a weak signal field is propagating inside a medium consisting of three level atoms. The strong control field is on resonance with the $e \rightarrow g^{\prime}$ transition, and a weak signal field is resonant with the $e \rightarrow g$ transition (see Fig. 1). In the absence of the control field, signal propagation is accompanied by strong absorption and dispersion. The situation changes when the control field is applied. The control field modifies properties of the medium. Absorption of signal light on resonance with the atomic transition is greatly reduced as a result of quantum interference. One can show that for a constant control field, the susceptibility of the medium for the weak probe field is equal to [40, 22]

$$
\chi(\Delta)=g^{2} N \frac{\gamma_{g g^{\prime}}+i \Delta}{\left(\gamma_{g g^{\prime}}+i \Delta\right)\left(\gamma_{g e}+i \Delta\right)+|\Omega|^{2}},
$$

where $\Delta$ is a detuning of the probe field from resonance, $g=d \sqrt{\frac{\omega_{0}}{2 \hbar \epsilon_{0} V}}$ is the atom-field coupling constant, $N$ is the total number of atoms in the sample, $\Omega$ is the Rabi frequency of the coupling field, $\omega_{0}$ is the frequency of the $g \rightarrow e$ transition, $\gamma_{i j}$ is the relaxation rate of the $i j$-coherence, and $V$ is the quantization volume.

Fig. 2 shows the real and imaginary part of refraction index as a function of detuning. Imaginary part determines transmission properties of the medium, whereas the real part is responsible for the refraction of light. When the control field is present the medium becomes transparent to the probe light on resonance with the $g \rightarrow e$ transition. The control field also changes the refraction index of the medium. As a result, the group velocity of the probe 


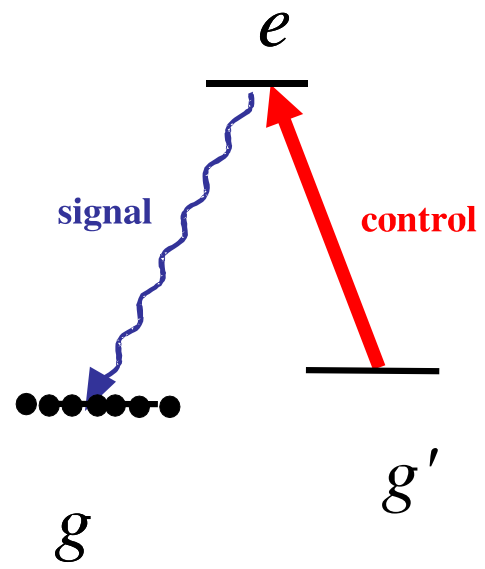

Figure 1: Scheme of atomic levels illustrating EIT process. Strong coupling field is on resonance with $e \rightarrow g^{\prime}$ transition, and a weak signal field is resonant with the $e \rightarrow g^{\prime}$ transition.

field $v_{g}$ can differ substantially from its vacuum value of $c$ :

$$
v_{g}=\frac{c}{1+g^{2} N / \Omega^{2}}
$$

and can be several orders of magnitude smaller than the speed of light in the vacuum.

The quantum mechanical description of the light propagation involves the solution of the Langevin equations for the signal field $\hat{\Phi}(z, t)=i \sum_{k} \hat{a}_{k} \exp \left(i\left(q z-w_{e} t\right)\right)$ and collective atomic coherence operator $\hat{S}_{s s^{\prime}}=\left(1 / N_{z}\right) \sum_{\mu=1}^{N_{z}} \sigma_{s s^{\prime}}^{\mu}$. Here $\sigma_{s s^{\prime}}^{\mu}=\left|s_{\mu}\right\rangle\left\langle s_{\mu}^{\prime}\right|$ is the $\mu^{\text {th }}$ atom hyperfine coherence operator, and $a_{k}$ is the annihilation operator for the $k$-th mode of the signal field. Assuming the adiabatic change of control field, solution of the Langevin equation is written in terms of the dark-state polariton operator [22]

$$
\hat{\Psi}(z, t)=\cos \theta \hat{\Phi}(z, t)+\sin \theta \hat{S}_{g g^{\prime}},
$$

where

$$
\begin{aligned}
& \cos ^{2} \theta=\frac{\Omega}{\Omega^{2}+g^{2} N} \\
& \sin ^{2} \theta=\frac{g^{2} N}{\Omega^{2}+g^{2} N}
\end{aligned}
$$



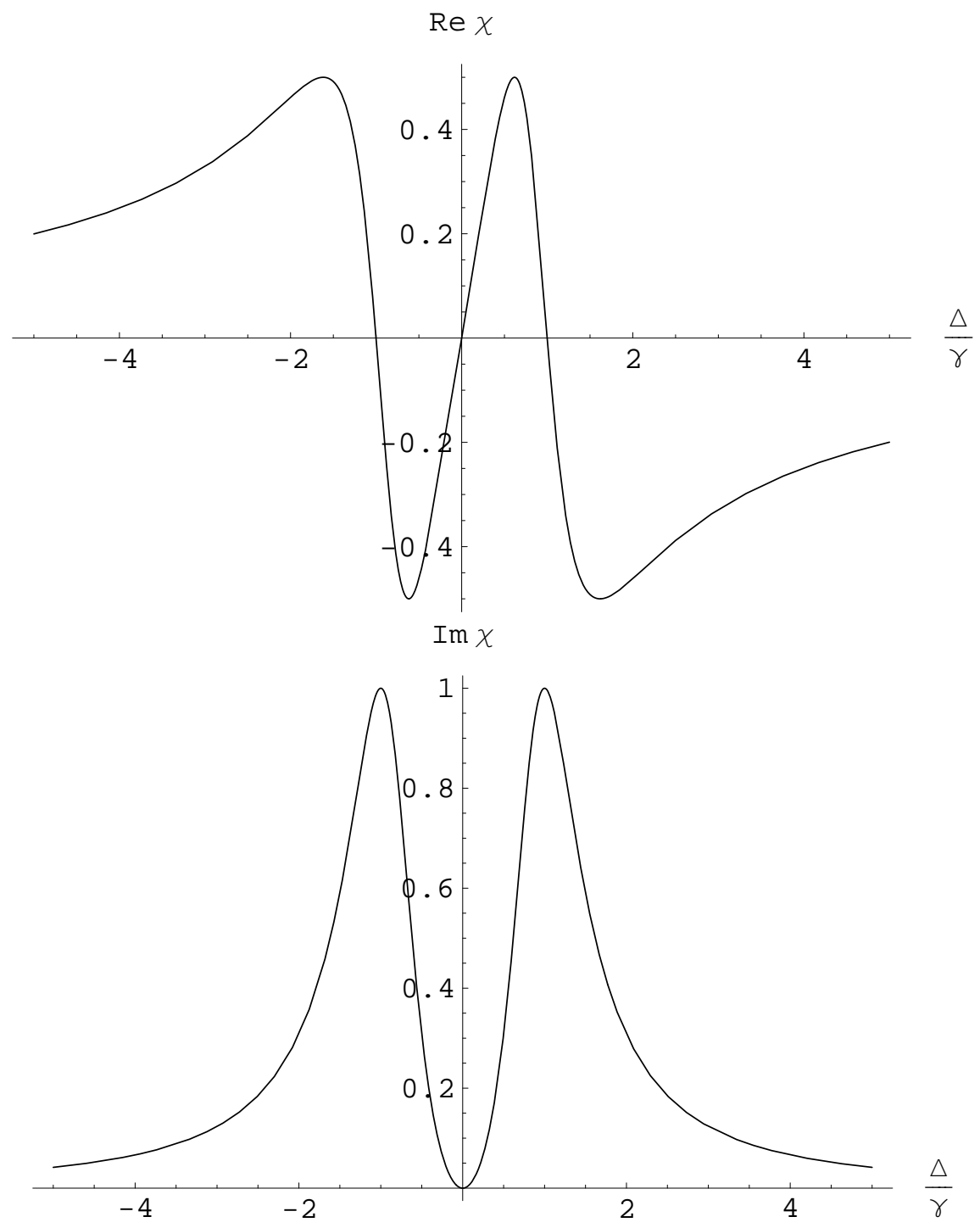

Figure 2: Real (top) and imaginary (bottom) parts of refraction index (a. u.) as a function of light detuning. We assume that $\gamma_{g g^{\prime}}=0$ and $\Omega / \gamma_{g e}=1$. 
and the dark state polariton operator obeys equation of motion

$$
\left(\frac{\partial}{\partial t}+c \cos ^{2} \theta \frac{\partial}{\partial z}\right) \hat{\Psi}(z, t)=0
$$

This equation describes propagation of a pulse with the group velocity $v_{g}=c \cos ^{2} \theta$. As the coupling field amplitude decreases, the atomic component of the dark state polariton increases and light component decreases. If the control beam is turned off, the group velocity of the pulse is reduced to zero and the quantum state of light is mapped onto the collective spin coherence. In the absence of external fields this coherence can be stored in the atomic ensemble for a long time. In order to read the quantum memory out, the control field is adiabatically switched back on. The state of atoms is then coherently mapped back to the state of light. Note that the concept of dark state polaritons can be generalized to the atoms with degenerate Zeeman sublevels [59, 60].

\subsection{DLCZ protocol for the generation of photon pairs}

The basic mechanism proposed in Ref. [11] involves entangling a single photon (signal) with a single collective excitation of an atomic ensemble via the Raman scattering of a weak write pulse. Figure 3A illustrates the DLCZ protocol of photon pair generation for a simple three level system. The Raman scattered photon in this case is uniquely correlated with a collective atomic state $S_{g g^{\prime}}^{\dagger}\left|0_{a}\right\rangle$. Here

$$
S_{g g^{\prime}}=\sum_{i=1}^{N}\left|g_{i}\right\rangle\left\langle e_{i}\right| e^{i \Delta \vec{k} \vec{r}_{i}},
$$

$\Delta \vec{k}=\vec{k}_{w}-\vec{k}_{s}$ is the difference between write and signal photon wavevectors, and $\vec{r}_{i}$ is the position of $i$-th atom.

The quantum state of the collective atomic mode can be written as

$$
|\phi\rangle=\left|0_{a}\right\rangle\left|0_{p}\right\rangle+\sqrt{p_{c}} S_{g g^{\prime}}^{\dagger} a^{\dagger}\left|0_{a}\right\rangle\left|0_{p}\right\rangle+O\left(p_{c}\right)
$$

Detection of the signal photon results in preparing a single spin excitation of the ensemble $S^{\dagger}\left|0_{a}\right\rangle$. Note that the state is exactly the same as the dark state polariton with the control field off. Therefore by applying second (read) pulse resonant to $e \rightarrow g^{\prime}$ transition, the 


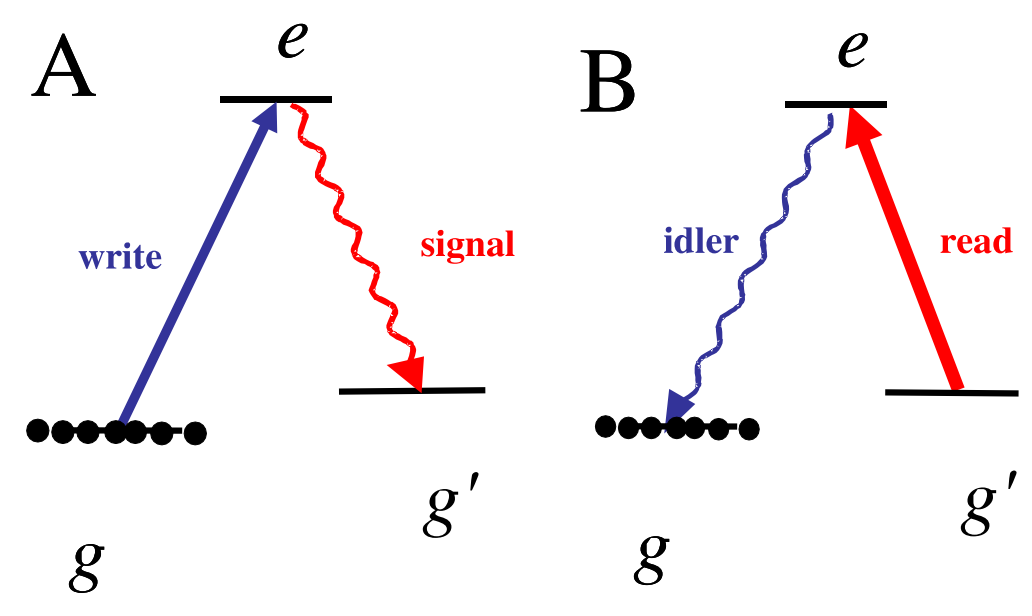

Figure 3: DLCZ protocol for the photon pair generation. Part A illustrates write process, Part B illustrates read process.

spin excitation can be converted into another (idler) photon (Fig. 3B). Due to collective enhancement, the second photon will be emitted in the direction determined by the phase matching condition: $\vec{k}_{s}+\vec{k}_{i}=\vec{k}_{r}+\vec{k}_{w}$. 


\section{CHAPTER II}

\section{STORAGE AND RETRIEVAL OF SINGLE PHOTONS TRANSMITTED BETWEEN REMOTE QUANTUM MEMORIES}

First part of this chapter is based on Ref. [48]. Second part describes additional details of the experiment including part of the Supporting Online Materials of Ref. [48].

\subsection{Motivation}

An elementary quantum network operation involves storing a qubit state in an atomic quantum memory node, and then retrieving and transporting the information through a single photon excitation to a remote quantum memory node for further storage or analysis. Implementations of quantum network operations are thus conditioned on the ability to realize such matter-to-light and/or light-to-matter quantum state mappings. In this Chapter, we report generation, transmission, storage and retrieval of single quanta using two remote atomic ensembles. A single photon is generated from a cold atomic ensemble at Site $A$ via the protocol of Duan, Lukin, Cirac, and Zoller (DLCZ) [11] and is directed to Site $B$ through a 100 meter long optical fiber. The photon is converted into a single collective excitation via the dark-state polariton approach of Fleischhauer and Lukin [42]. After a programmable storage time the atomic excitation is converted back into a single photon. This is demonstrated experimentally, for a storage time of 500 nanoseconds, by measurement of an anticorrelation parameter $\alpha$. Storage times exceeding ten microseconds are observed by intensity cross-correlation measurements. The length of the storage period is two orders of magnitude longer than the time to achieve conversion between photonic and atomic quanta.

A quantum network, consisting of quantum nodes and interconnecting channels, is an 
outstanding goal of quantum information science. Such a network could be used for distributed computing or for the secure sharing of information between spatially remote parties $[3,6,7,2,61,11]$. While it is natural that the network's fixed nodes (quantum memory elements) could be implemented by using matter in the form of individual atoms or atomic ensembles, it is equally natural that light fields be used as carriers of quantum information (flying qubits) using optical fiber interconnects. The matter-light interface seems inevitable since the local storage capability of ground state atomic matter cannot be easily recreated with light fields. Interfacing material quanta and single photons is therefore a basic primitive of a quantum network.

The potential of atomic ensembles to serve as quantum memories has recently attracted considerable attention $[62,22,42,11,63,39]$. Using the physics of "slow light" propagation in an optically thick atomic ensemble, weak coherent laser pulses have been stopped and retrieved in a controlled fashion $[42,41,26,27]$.

\subsection{Experiment}

Here we report the demonstration of the generation, transmission, storage and retrieval of single photons using remote atomic ensembles as quantum memories. The essential ingredient which we report here, is the ability to convert single photons into single collective atomic excitations. In our experiment the remote quantum memories are based on cold atomic clouds of ${ }^{85} \mathrm{Rb}$ confined in magneto-optical traps (MOTs) at Sites $A$ and $B$, as shown in Fig. 4. Sites $A$ and $B$ are physically located in adjacent laboratories, with a 100 meter long single-mode optical fiber serving as the quantum information channel.

Our protocol begins with the generation of single photons at Site $A$, using the DLCZ approach in the off-axis, counter-propagating geometry $[54,47]$. The fiber channel directs the signal field to Site $B$ where an optically thick atomic ensemble is prepared in level $|b\rangle$ (right inset in Fig. 4). The signal field propagation in the atomic medium is controlled by an additional laser field (control) through the process of electromagnetically-induced transparency (EIT) [40, 64]. As we deal with an unpolarized atomic ensemble, we must take into account the Zeeman degeneracy of the atomic levels. Choosing the same circular 


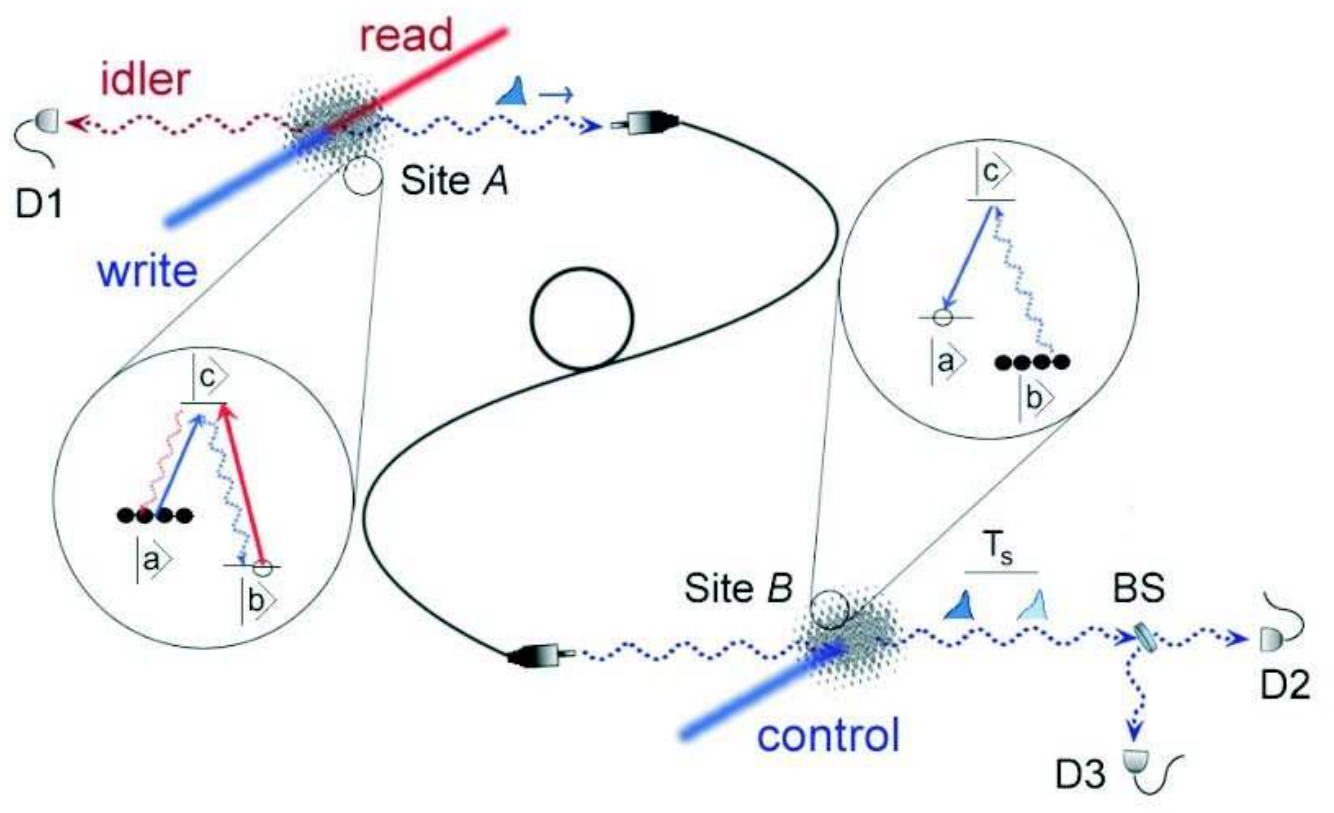

Figure 4: A schematic diagram of our experimental setup demonstrating generation, transmission, storage and retrieval of single photon excitations of the electromagnetic field. Two atomic ensembles at Sites $A$ and $B$ are connected by a single-mode fiber. The insets show the structure and the initial populations of atomic levels for the two ensembles. All the light fields responsible for trapping and cooling, as well as the quadrupole magnetic fields in both MOTs, are shut off during the period of the protocol. The ambient magnetic field at each Site is compensated by three pairs of Helmholtz coils (not shown). Correlated signal and idler fields are generated at Site $A$. The signal field is transmitted via optical fiber from Site $A$ to Site $B$, where it is converted to atomic excitation, stored for a duration $T_{s}$, and subsequently retrieved. A Hanbury Brown-Twiss setup consisting of a beamsplitter BS and two detectors D2 and D3, together with detector D1 for the idler field, are used to verify the single photon character of the retrieved field. 


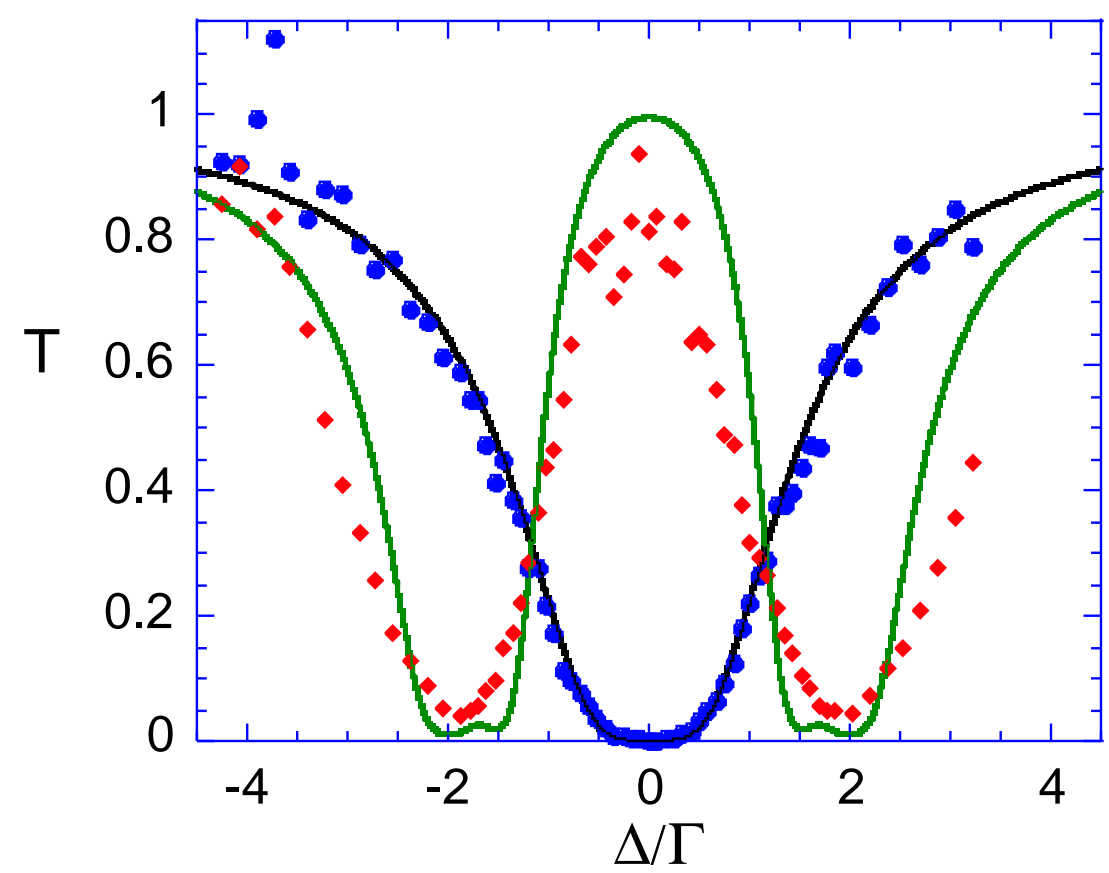

Figure 5: Measured transmission spectra of a coherent probe field as the function of probe detuning in the presence of, and absence of, EIT. Data are taken using $700 \mathrm{~ns}$ long coherent laser pulses. $T$ is the intensity transmittance, $\Delta$ is the probe detuning and $\Gamma$ is the decay rate of level $|c\rangle$. In the absence of control field (circles) the probe is strongly absorbed near resonance, whereas with the control field on (diamonds) the medium becomes transparent. Each probe pulse contains on average 0.3 photons. Each data point is an average of $2 \times 10^{5}$ experimental trials. The optical thickness $d=8$ and the control field Rabi frequency $\Omega=3 \Gamma$ are used to obtain the solid curves, based on the theoretical model discussed in this chapter.

polarizations for both the probe and the control fields allows us to retain transparency. In Fig. 5 we show the EIT transmission spectrum recorded for a coherent laser probe field instead of the signal field. Evidently, in the absence of the control light the probe field is absorbed by the optically thick sample. With the addition of the cw control field, the medium is rendered transparent around the $|b\rangle \leftrightarrow|c\rangle$ transition resonance $\Delta=0$.

The control field strongly modifies the group velocity of the signal field. For a timedependent control field, a strong reduction of the group velocity of the propagating signal field can be understood in terms of a coupled matter-light field excitation known as a "darkstate polariton." By adiabatically switching off the control field, the coupled excitation can be converted into a pure atomic excitation, i.e., the signal field is "stopped" [42, 26, 27]. An important condition to achieve storage is a sufficiently large optical thickness of the atomic 
sample, which enables strong spatial compression of the incident signal field [22]. In our experiment the measured optical thickness $d \simeq 8$. Fig. 6 compares our observations with the predictions of a theoretical model described below. Fig. 6a compares the propagation of the signal pulse in vacuum and in the atomic medium under conditions of EIT with a cw control field. The observed pulse delay under conditions of EIT is about $20 \mathrm{~ns}$, corresponding to more than three orders of magnitude reduction in group velocity. Fig. $6 \mathrm{~b}$ shows the effect of turning off the control-storage field when the signal pulse is approximately centered in the medium, and the subsequent retrieval of the signal field when the control-retrieval field is switched back on after a 500 ns storage time. Fig. 6c shows retrieval after a storage time of 15 $\mu \mathrm{s}$. Qualitative agreement of the pulse shapes has been obtained in our theoretical analysis of the protocol using the full Zeeman structure of the atoms and a classical description of the signal field (Fig. 6d-f).

In order to verify the single-photon character of the signal field (a) without storage, and (b) with storage and retrieval, we use a Hanbury Brown-Twiss detection scheme, employing a beamsplitter followed by two single photon counters, as shown in Fig. 4 [65]. To provide such characterization, we note that classical fields must satisfy a criterion $\alpha \geq 1$ based on the Cauchy-Schwarz inequality [65, 66]. For an ideally prepared single photon state $\alpha \rightarrow 0$. Here the anticorrelation parameter $\alpha$ is a function of the storage time $T_{s}$, and is given by the ratio of various photoelectric detection probabilities which are measured by the set of detectors D1,D2 and D3 (described in the next section):

$$
\alpha\left(T_{s}\right)=\frac{p_{1} p_{123}}{p_{12} p_{13}}
$$

As an auxiliary measure of signal-idler field correlations, and as a way to quantify the quantum memory storage time, we also evaluate the normalized intensity cross-correlation function $g_{s i} \equiv\left(p_{12}+p_{13}\right) /\left[p_{1}\left(p_{2}+p_{3}\right)\right][67,30]$. In particular, it serves to estimate the total efficiency and background levels in the experiment, since $g_{s i}$ is, by definition, independent of efficiencies whereas $p_{1}$ is proportional to the overall idler channel efficiency.

First we measure $g_{s i}$ and $\alpha$ without storage at Site $B$ (i.e., with no atomic sample in place), and the results are displayed in Fig. 7, a and b, respectively. Next we add an 

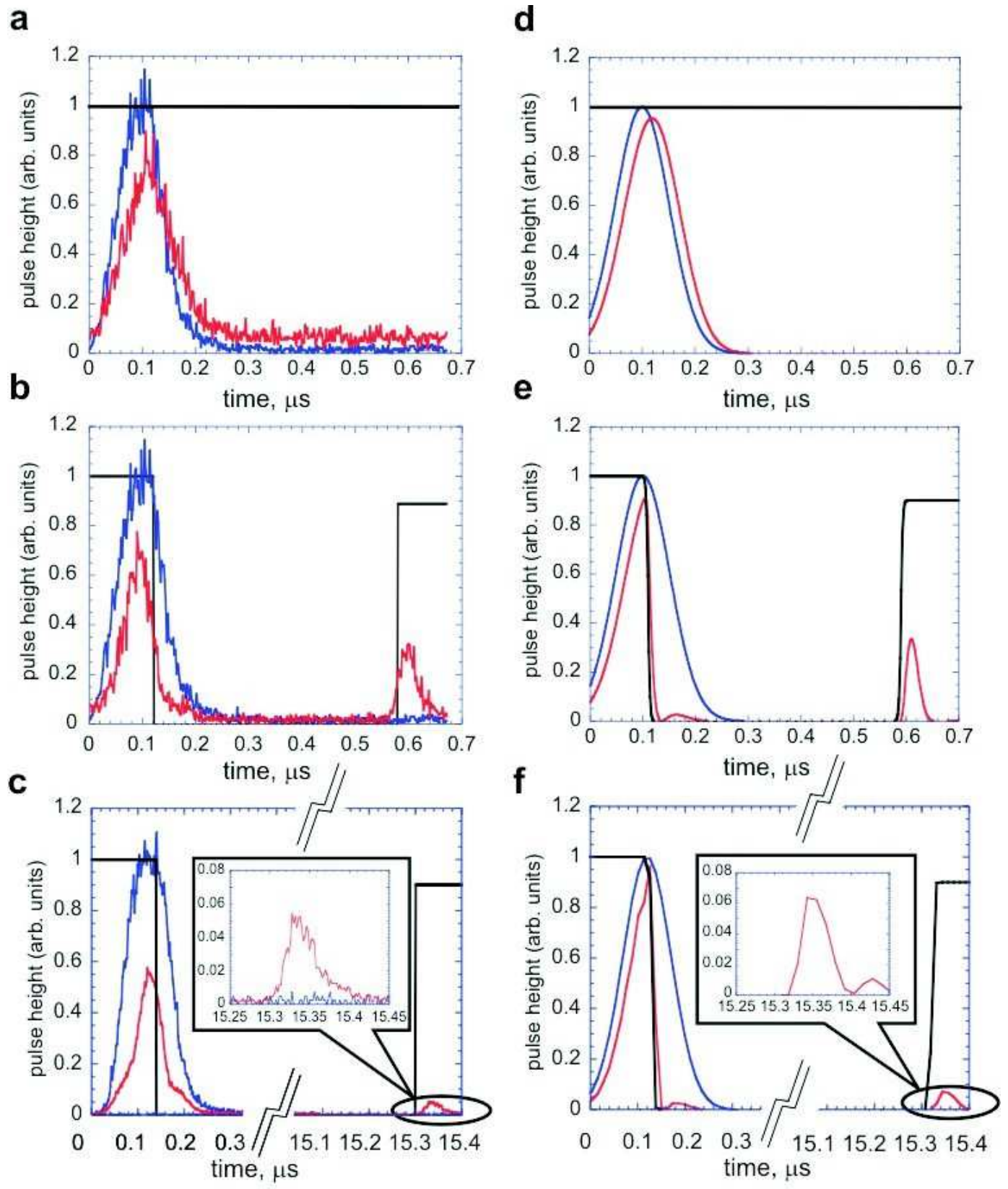

Figure 6: Experimental and theoretical pulse shapes as a function of time, showing EIT, storage and retrieval. The color code is: control field - black, pulse in vacuum - blue, delayed, stored and retrieved field - red. Panel (a) with a cw control field shows EIT pulse delay. In panel (b) the control field is switched off and then on again after $500 \mathrm{~ns}$, shows light storage and retrieval. Panel (c) is similar to (b) but with a $15 \mu$ s storage. Panels (d), (e), and (f) are corresponding theoretical plots. 

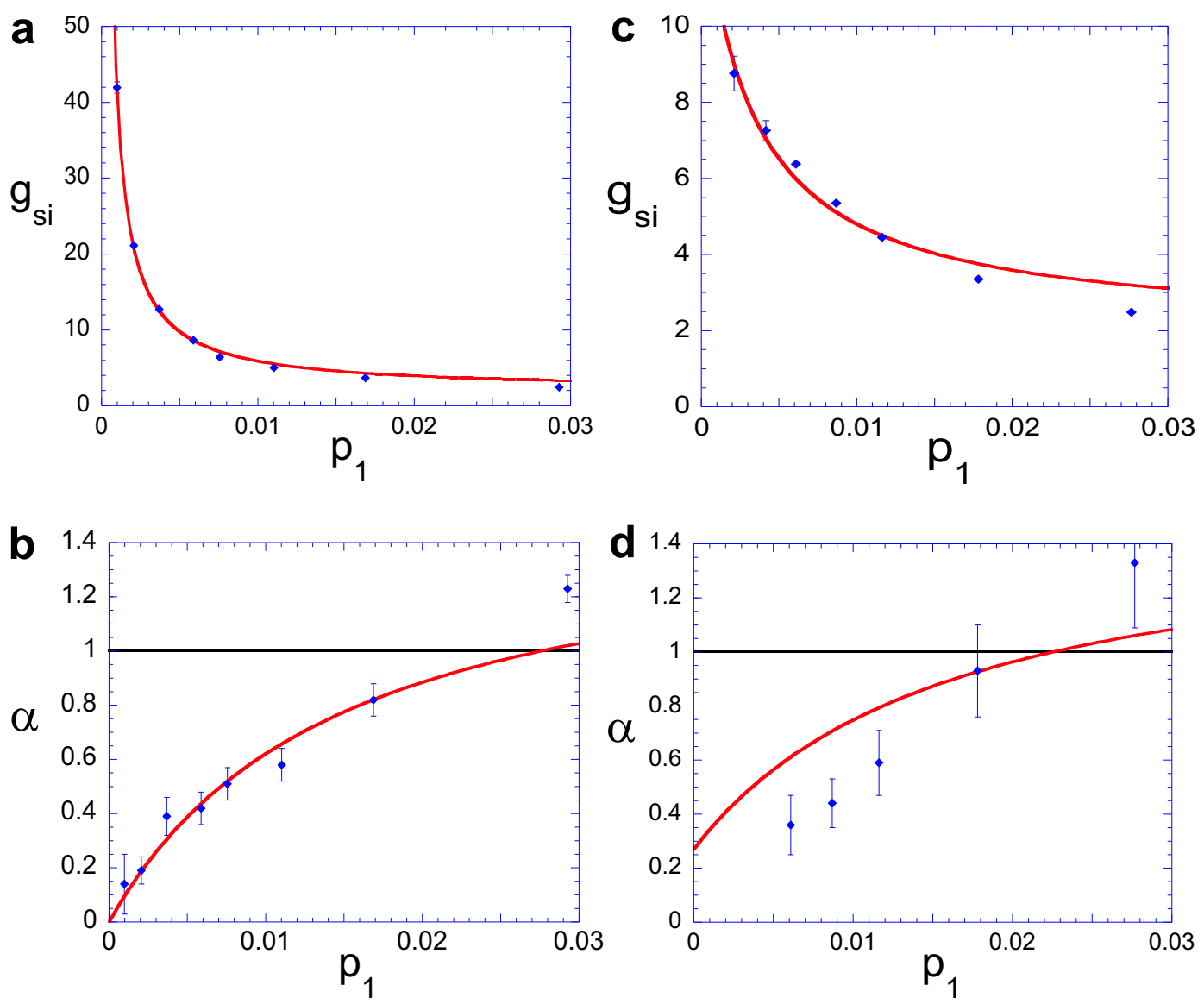

Figure 7: Measured intensity cross-correlation function $g_{s i}$ and anticorrelation function $\alpha$ as a function of the idler photoelectric detection probability $p_{1}$. Panels (a) and (b) are for the source (propagation in vacuum). Panels (c) and (d) are for stopped, stored for $500 \mathrm{ns,}$ and retrieved signal field. The solid lines are based on a theoretical model that includes losses and background. Error bars represent \pm one standard deviation and are based on the statistics of the photoelectric counting events. 
optically thick atomic sample at Site $B$, and perform storage of duration $T_{s}=500 \mathrm{~ns}$ and subsequent retrieval of the signal field, with results shown in Fig. 7, c and d, respectively. No correction for background or dark counts were made to any of the experimental counting rates. The curve fits of $g_{s i}$ are based on a simple theoretical model, and allow us to obtain the efficiency in the idler channel and the background contributions to $p_{2}$ and $p_{3}$ for the stored signal field. These same values are used to produce the corresponding theoretical curves in Fig. 7, b and d. The measured values of $\alpha<1$, displayed in Fig. 7, b and d, confirm the single-photon character of both the source and retrieved signal fields (with the minimum values of $\alpha=0.14 \pm 0.11$ and $\alpha=0.36 \pm 0.11$, respectively). Overall, we estimate that the probability $p_{s}$ for successful generation, transmission, storage, retrieval, and detection of a signal photon is approximately $p_{s} \simeq 10^{-5}$ for each trial. The efficiency of photon storage and retrieval $E$ can be estimated as the ratio of the values of $p_{2}+p_{3}$ with and without storage. We find $E \simeq 0.06$, in agreement with the theoretical result shown in Fig.6e.

To investigate the storage capability of our quantum memory at Site $B$, we measure $g_{s i}$ as a function of the storage time of the signal field $T_{s}$ (Fig. 8). A Gaussian fit provides a time constant $\tau=11 \mu \mathrm{s}$, which is an estimate of our quantum memory time. The collapse is consistent with the Larmor precession of a dark-state polariton in an unpolarized atomic ensemble in a residual magnetic field [43, 46]. Experimentally we attempt to null the uniform, dc component of the magnetic field. A definitive way to distinguish whether the collapse is due to uniform or non-uniform and ac fields is to measure the damping time of the periodic revivals of the retrieved signal field at longer storage times. In a uniform magnetic field, undamped revivals of the dark-state polariton should occur at times equal to $n T_{L}$, where $T_{L}$ is the Larmor period for level $|a\rangle$ or $|b\rangle$ and $n$ can be either integer or half-integer, depending on the direction of the magnetic field relative to the light beam geometry (the full theory is presented in Ref.[59,60]). We have conducted separate experiments with an externally applied magnetic field [49] (see also Chapter 3), which suggest that the collapse in the present experiment is likely due to magnetic field gradients and/or ac fields at the level of a few tens of $\mathrm{mG}$. However, more extensive investigations to quantitatively determine the 


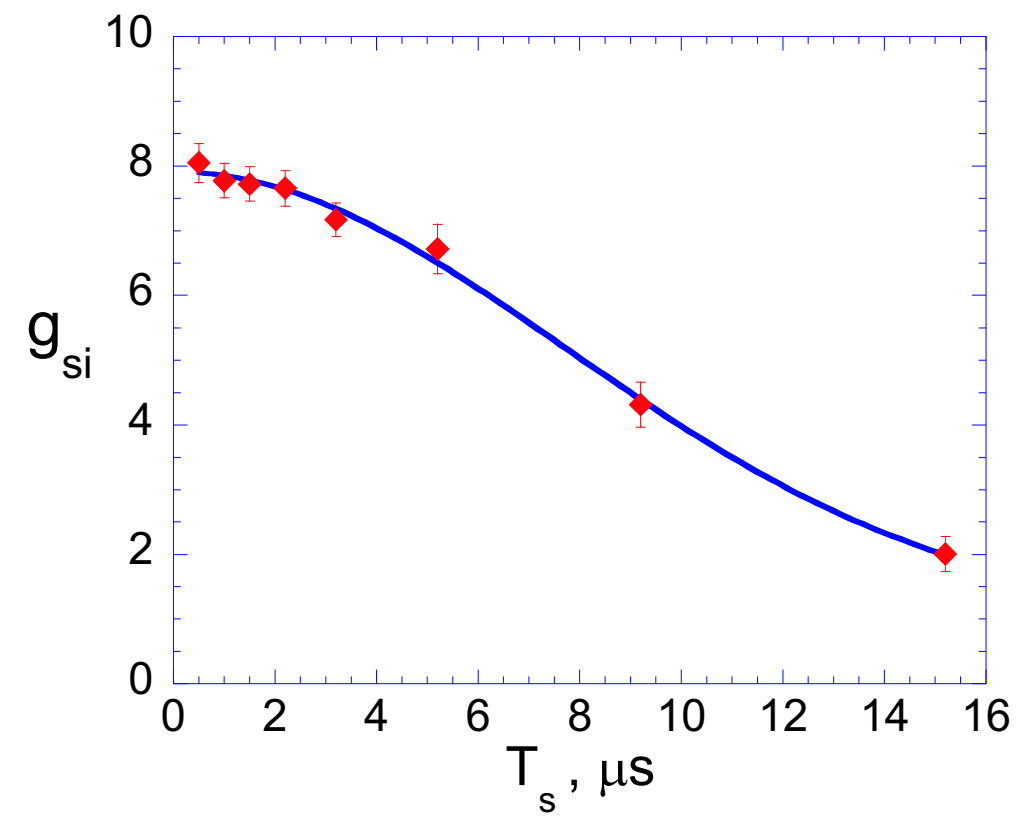

Figure 8: Normalized signal-idler intensity correlation function $g_{s i}$ as a function of the storage time $T_{s}$ at Site $B$. Data (diamonds) are taken for $p_{1}=0.0047$, but with a smaller background contribution than that of Fig.4, c and d. The full curve is a fit of the form $1+$ $B \exp \left(-t^{2} / \tau^{2}\right)$ with $B=7$ and the collapse time $\tau=11 \mu$ s as adjustable parameters. Error bars represent \pm one standard deviation and are based on the statistics of the photoelectric counting events. 
temporal and spatial structure of the residual magnetic field, and the various contributions to it, are required.

We have demonstrated generation, storage and retrieval of single quanta transmitted between two remote atomic ensembles serving as quantum memory elements. The control of the matter-field interface at the level of single quanta, and at remote sites, is encouraging for further developments and applications in quantum information science. In particular, the storage of a photonic qubit, with two logical states, would represent a crucial advance. In order to achieve this, the quantum memory at Site $B$ would likewise need a second logical state, so as to realize a collective atomic qubit. Two different approaches for such qubits are described in this thesis (See Chapters 5 and 6 , and $[46,47]$ ). Addition of the second logical state to both quantum memories at Sites $A$ and $B$ makes is possible to generate remote entanglement of two atomic qubits. The corresponding experiment is described in Chapter 7.

\subsection{Measurement procedure}

To generate single photons at Site $A$, we use the DLCZ approach in the off-axis, counterpropagating geometry introduced by Harris and coworkers [54]. The insets in Fig. 4 indicate schematically the structure of the three atomic levels involved, $|a\rangle,|b\rangle$ and $|c\rangle$, where $\{|a\rangle ;|b\rangle\}$ correspond to the $5 S_{1 / 2}, F=\{3,2\}$ levels of ${ }^{85} \mathrm{Rb}$, and $|c\rangle$ represents the $\left\{5 P_{1 / 2}, F=3\right\}$ level associated with the $D_{1}$ line at $795 \mathrm{~nm}$. The experimental sequence begins with an unpolarized sample of atoms prepared in level $|a\rangle$ (left inset of Fig. 4). A $160 \mathrm{~ns}$ long write laser pulse tuned to the $|a\rangle \rightarrow|c\rangle$ transition is focused into the MOT with a Gaussian waist of about $400 \mu \mathrm{m}$. The write pulse generates a cone of forward Ramanscattered signal field via the $|c\rangle \rightarrow|b\rangle$ transition. We collect a Gaussian mode centered

around the momentum $\vec{k}_{s}$ that forms an angle of about $2^{\circ}$ with the write beam. The write pulse is so weak that on average less than one photon is scattered into the collected mode for each pulse. The signal field is coupled into the 100 meter long fiber connecting Sites $A$ and $B$.

For each signal photon emission event, a correlated collective atomic excitation is created 
in the atomic ensemble. After a delay $\Delta t=200 \mathrm{~ns}$, a $140 \mathrm{~ns}$ long counter-propagating read laser pulse resonant with the $|b\rangle \rightarrow|c\rangle$ transition illuminates the atomic ensemble and converts the atomic excitation into the idler field. Under the conditions of collective enhancement, the idler field is emitted with high probability into the mode determined by the phase-matching condition $\vec{k}_{i}=\vec{k}_{w}+\vec{k}_{r}-\vec{k}_{s}$, where $\vec{k}_{i}, \vec{k}_{w}$ and $\vec{k}_{r}$ are the wave vectors of the idler, write and read fields, respectively. The waist of the signal-idler mode in the MOT is about $150 \mu \mathrm{m}$. The idler field is directed onto a single photon counter D1. Ideally, photoelectric detection of the idler field projects the quantum state of the signal field into a single photon state. The repetition rate of the experiment is $2 \cdot 10^{5} \mathrm{~s}^{-1}$. Each data point in Fig.7 involves an average over a time period that varied from several minutes up to 1.5 hours for the data point with the lowest value of $p_{1}$ in $\mathrm{d}$.

To measure the photoelectric detection probabilities $p_{1}, p_{2}, p_{3}, p_{13}, p_{12}, p_{23}$, and $p_{123}$, the outputs of the detectors are fed to three "Stop" inputs of the time-interval analyzer which records the arrival times with a 2 ns time resolution. The electronic pulses from the detectors D1,D2,D3 are gated for periods $\left[t_{0}^{i}, t_{0}^{i}+T_{g}^{i}\right]$, with $T_{g}^{1}=140 \mathrm{~ns}, T_{g}^{2}=T_{g}^{3}=240$ ns, respectively, centered on the times determined by the write and read (for no storage) or control-retrieval (for storage) laser pulses. Counts recorded outside the gating periods are therefore removed from the analysis. The list of recorded events allows us to determine the single-channel photoelectric event probabilities $p_{i}=N_{i} / M$, where $N_{i}$ is the total number of counts in the $i$-th channel and $\mathrm{M}$ is the number of experimental trials, (for $\mathrm{D} i, i=1,2,3)$. If photoelectric detections in different channels $i, k, m$ happen within the same gating period, they contribute to the corresponding joint probabilities $p_{i j}=N_{i j} / M$, where $N_{i j}$ is the total number of coincidences between $\mathrm{D} i$ and $\mathrm{D} j, i, j=1,2,3$. The joint probability of all three detectors registering a count is given by $p_{123}=N_{123} / M$.

\subsection{Photoelectric counting statistics}

Here we provide details of the analysis of the photoelectron counting statistics of the light fields detected in our experiment, in support of generation, storage, and retrieval of single photon states of the electromagnetic field. 
In order to take into account the possibility of sequences of photon pairs, we use a theoretical model based on parametric down-conversion, in which the annihilation operators for the idler and signal field are transformed as [30]:

$$
\begin{aligned}
& \hat{a}_{i}^{(\text {out })}=\cosh (\eta) \hat{a}_{i}^{(\text {in })}+\sinh (\eta) \hat{a}_{s}^{\dagger(\text { in })} \\
& \hat{a}_{s}^{(\text {out })}=\cosh (\eta) \hat{a}_{s}^{(i n)}+\sinh (\eta) \hat{a}_{i}^{\dagger(\text { in })}
\end{aligned}
$$

Here $\eta$ is the Raman gain at Site $A$. We also wish to assess the overall efficiencies and the background levels in our experiment. Modelling the background to the signal in terms of a coherent field with average photon number $B_{s}$, we find that

$$
g_{s i}=\frac{\left(1+2 \sinh ^{2}(\eta)\right)+B_{s}}{\sinh ^{2}(\eta)+B_{s}} .
$$

We also determine the anticorrelation parameter $\alpha$ of Grangier et al. [65]:

$$
\alpha=\frac{\sinh ^{2}(\eta)\left(4+6 \sinh ^{2}(\eta)\right)+4 B_{s}\left(1+2 \sinh ^{2}(\eta)\right)}{\left(1+2 \sinh ^{2}(\eta)+B_{s}\right)^{2}} .
$$

The singles count rates at detectors D1, D2, and D3 are given by $R_{1}=\epsilon_{1} \sinh ^{2}(\eta), R_{2}=$ $|T|^{2} \epsilon_{2} \sinh ^{2}(\eta)$ and $R_{3}=|R|^{2} \epsilon_{3} \sinh ^{2}(\eta)$ (assuming that $R_{i} \ll W$, where $W$ is the repetition rate of the experiment); $T$ and $R$ are the transmission and reflection coefficients of the beamsplitter BS, shown in Fig. 9.

In the absence of the medium we empirically find negligible background $B_{s}$. The solid curve in Fig. 7A is based on this model, setting $B_{s}=0$. We find that the best fit to the data in Fig. 7A is given by $\epsilon_{1} \approx 0.039$. The solid line in Fig. 7B is based on Eq. 12 with this value of $\epsilon_{1}$.

For the stored light, we have to account for the fact that a significant fraction of detected signal photons are due to background associated with the control-retrieval pulse. By fitting the data of Fig. $7 \mathrm{C}$ to Eq.11, we find $B_{s} \approx 0.08$. Substituting this value into Eq.12, we obtain the solid curve in Fig. 7D. In order to reduce this background, we have performed initial investigations using an optically pumped $\mathrm{Rb}$ cell to filter out light at the frequency of the control field. In this case we found increased non-classical correlations between the idler and the stored and retrieved signal photon, e.g., for $T_{s}=500 \mathrm{~ns} g_{s i}$ increased from $8 \pm 0.2$ to $15.6 \pm 1.4$ 


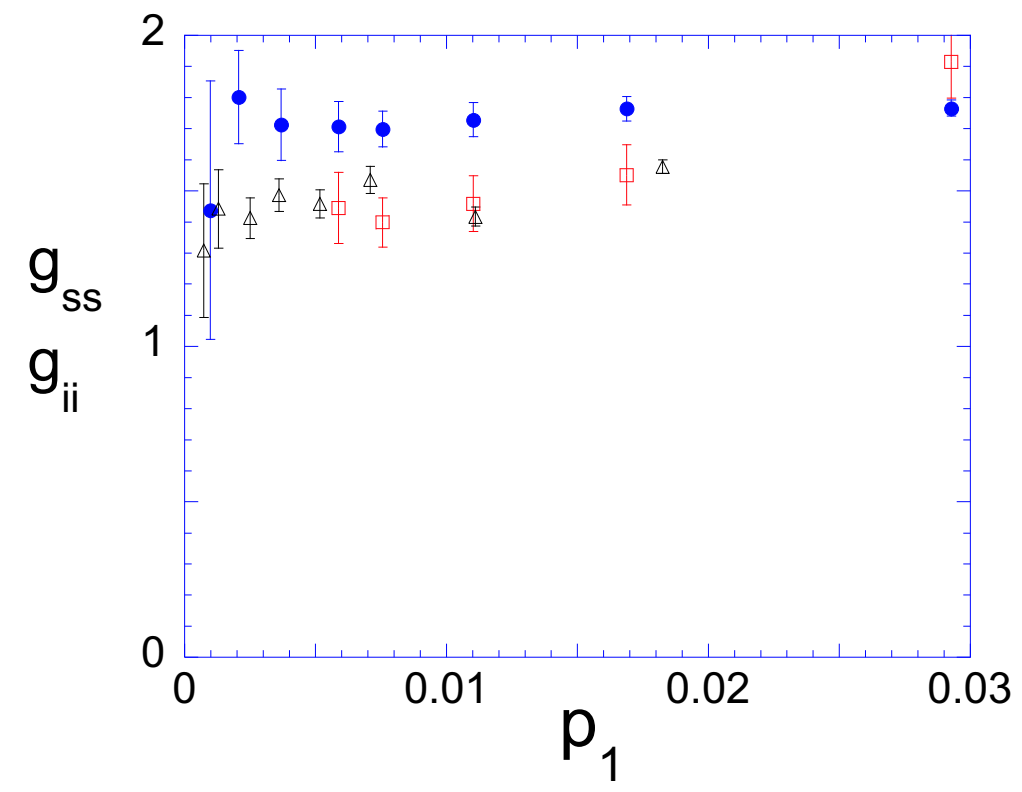

Figure 9: Normalized intensity autocorrelation functions $g_{i i}$ (triangles) and $g_{s s}$ (circles for the source, squares for the stored and retrieved field). Uncertainties are based on the statistics of the photon counting events.

In addition, we measure the intensity autocorrelation functions $g_{s s}=p_{23} /\left[p_{2} p_{3}\right]$ and $g_{i i}$. These are shown in Fig. 9. In order to evaluate the latter, we insert a beamsplitter and additional detector $D_{a}$ into the path of the idler photon, so that $g_{i i}=p_{1 a} /\left[p_{1} p_{a}\right]$. Using these together with the measured values of $g_{s i}$ shown in Fig. 4 of Ref.[48], one can evaluate Clauser's parameter $R=g_{s i}^{2} /\left[g_{s s} g_{i i}\right]$. For classical fields $R \leq 1$, whereas we observe strong violation of this inequality. The Clauser parameter $R=g_{s i}^{2} /\left[g_{s s} g_{i i}\right]$ as a function of photodetection probability $p_{1}$ is shown in Fig. 10 for our single photon source, and in Fig. 11 after storage.

The total measured transmission and detection efficiencies for the idler and signal fields respectively are $w_{i}=0.25 \pm 0.03$ and $w_{s}=0.15 \pm 0.02$, consisting of the quantum efficiencies of the detectors $0.55 \pm 0.05$ and the passive transmission losses accounting for the rest. The ratio of $h \equiv \epsilon_{i} / w_{i}=0.16$ indicates the strength of the spatial signal-idler correlations in our source of conditional single photons at Site $A$, with $h \rightarrow 1$ for the ideal case. 


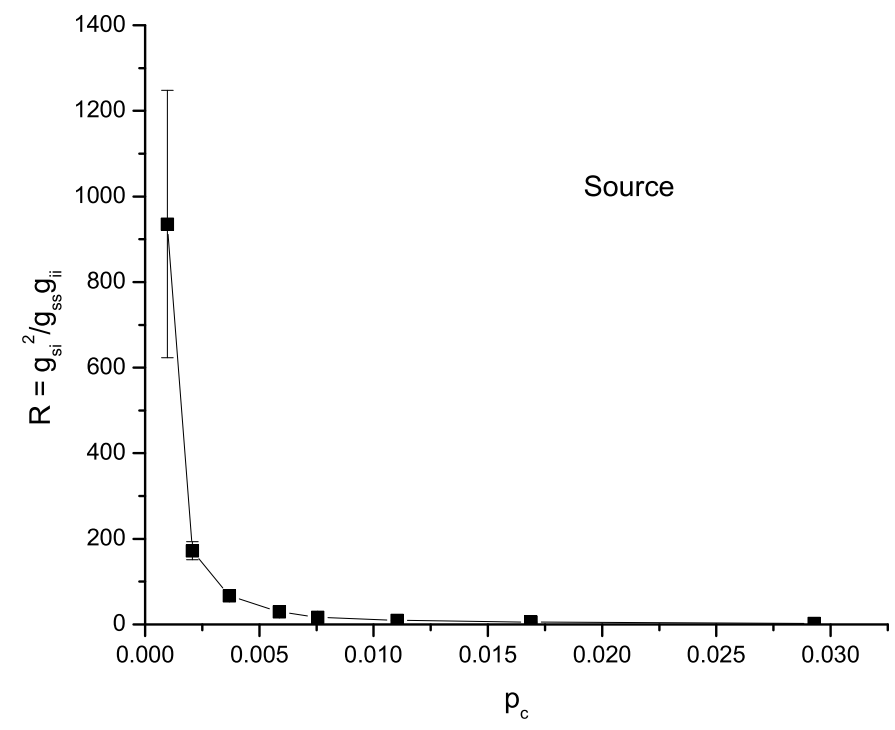

Figure 10: Clauser parameter $R=g_{s i}^{2} /\left[g_{s s} g_{i i}\right]$ as a function of photodetection probability $p_{1}$ for the single photon source.

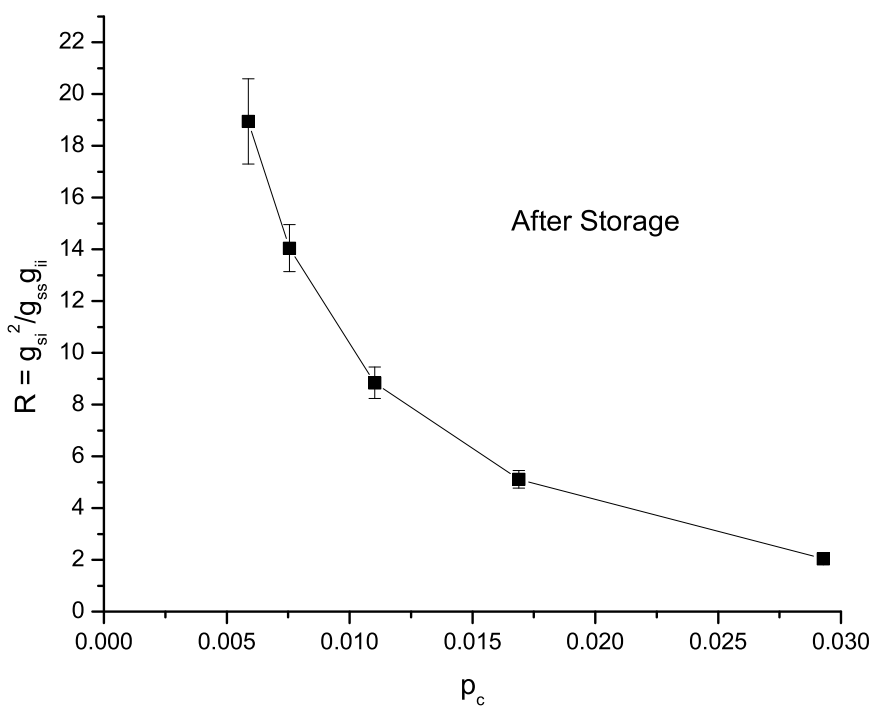

Figure 11: Clauser parameter $R=g_{s i}^{2} /\left[g_{s s} g_{i i}\right]$ as a function of photodetection probability $p_{1}$ after 500 ns storage time. 


\section{CHAPTER III}

\section{OBSERVATION OF DARK STATE POLARITON COLLAPSES AND REVIVALS}

This chapter is based on Ref. [49].

\subsection{Motivation}

Atomic ensembles show significant promise as quantum memory elements in a quantum network $[2,61,11,23,22]$. A "dark-state polariton" is a bosonic-like collective excitation of a signal light field and an atomic spin wave [42], whose relative amplitude is governed by a control laser field. In the context of quantum memories, the dark state polariton should enable adiabatic transfer of single quanta between an atomic ensemble and the light field. Seminal "stopped-light" experiments that used laser light excitation [26, 27, 68] can be understood in terms of the dark-state polariton concept. In a recent work the storage and retrieval of single photons using an atomic ensemble based quantum memory was reported, and the storage time was conjectured to be limited by inhomogeneous broadening in a residual magnetic field [48].

During storage, the dark-state polariton consists entirely of the collective spin wave excitation. According to the dark-state polariton concept, the retrieved signal field should exhibit the collapse and revivals experienced by the spin wave.[60] The revivals occur at integer multiples of one half the Larmor period, with dynamics that are sensitive to the relative orientation of the magnetic field and the light wavevector. The spin wave part of the dark-state polariton involves a particular superposition of atomic hyperfine coherences (see Eq.(1) below), intimately related to the phenomenon of electromagnetically-induced

transparency (EIT) [40, 64]. Revivals of single atom coherences were observed in atom interferometery $[69,70]$. Coupled exciton-polariton oscillations in semiconductor microcavities have also been reported $[71,72]$. Evolution of the dark-state polariton for the simple 
three level atomic system in a magnetic field was reported in [68].

The remarkable protocol of Duan, Lukin, Cirac, and Zoller (DLCZ) provides a measurementbased scheme for the creation of atomic spin excitations [11]. In systems where EIT is operative, these excitations will in general contain a dark-state polariton component. The orthogonal contribution may be regarded as a bright-state polariton in that it couples dissipatively to the excited atomic level in the presence of the control field [73]. Observation of the retrieved signal field, however, picks out the dark state polariton part, while the orthogonal component is converted into spontaneous emission [60]. A number of previous works reported generation and subsequent retrieval of DLCZ collective excitations $[43,44,52,45,46,54,47,74]$. Several of these studies investigated the decoherence of these excitations in cold atomic samples $[43,45,46,47,74]$. It has been similarly conjectured in these works that the decay of the coherence was due to spin precession in the ambient magnetic field. While the observed decoherence times are consistent with the residual magnetic fields believed to be present, the observation of revivals predicted in Ref.[60] would be solid proof that Larmor precession is indeed the current limitation on the quantum memory lifetime. Moreover, controlled revivals could provide a valuable tool for quantum network architectures that involve collective atomic memories [11, 23, 22].

\subsection{Experiment}

We describe in this Chapter observations of collapses and revivals of dark-state polaritons in agreement with the theoretical predictions [60]. In our experiment, we employ two different sources for the signal field, a coherent laser output and a conditional source of single photons [11]. The latter is achieved by using a cold atomic cloud of ${ }^{85} \mathrm{Rb}$ at Site $A$ in the off-axis geometry pioneered by Harris and coworkers [54]. Another cold atomic cloud of ${ }^{85} \mathrm{Rb}$ at Site $B$ serves as the atomic quantum memory element, as shown in Fig. 12. Sites $A$ and $B$ are physically located in adjacent laboratories connected by a 100 meter long single-mode optical fiber. The fiber channel directs the signal field to the optically thick atomic ensemble prepared in level $|b\rangle$. The inset in Fig. 12 indicates schematically the structure of the three atomic levels involved, $|a\rangle,|b\rangle$ and $|c\rangle$, where $\{|a\rangle ;|b\rangle\}$ correspond to 


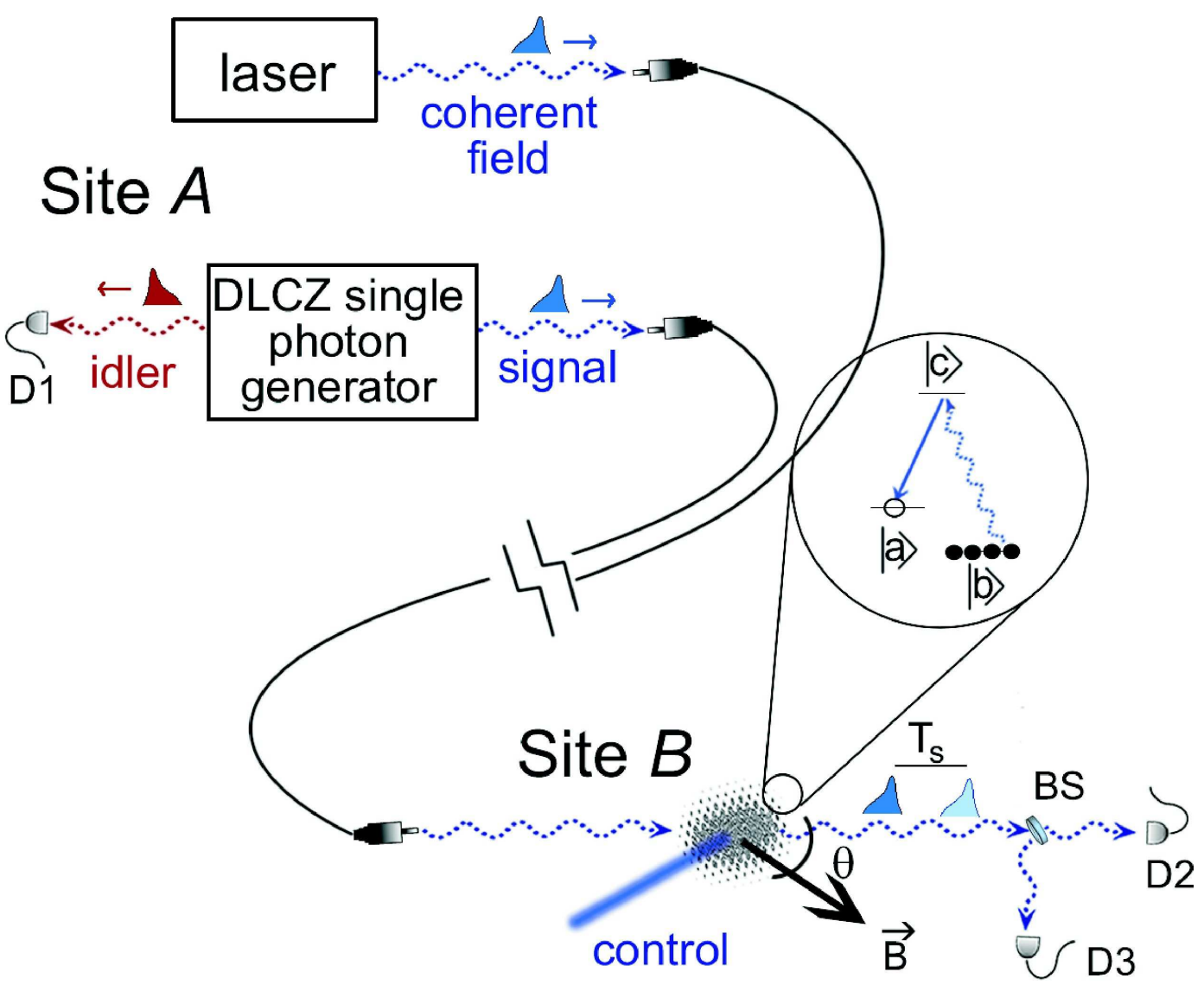

Figure 12: A schematic diagram illustrates our experimental setup. A signal field from either a laser, or a DLCZ source of conditional single photons at Site $A$ is carried by a single-mode fiber to an atomic ensemble at Site $B$, where it is resonant on the $|b\rangle \leftrightarrow|c\rangle$ transition. The signal field is stored, for a duration $T_{s}$, and subsequently retrieved by timedependent variation of a control field resonant between levels $|a\rangle$ and $|c\rangle$. All the light fields responsible for trapping and cooling, as well as the quadrupole magnetic field in the MOT, are shut off during the period of the storage and retrieval process. An externally applied magnetic field created by three pairs of Helmholtz coils (not shown) makes an angle $\theta$ with the signal field wavevector. The inset shows the structure and the initial populations of atomic levels involved. The signal field is measured by detectors D2 and D3, while detector D1 is used in the conditional preparation of single photon states of the signal field at Site $A$. 
the $5 S_{1 / 2}, F_{a}=3, F_{b}=2$ levels of ${ }^{85} \mathrm{Rb}$, and $|c\rangle$ represents the $5 P_{1 / 2}, F_{c}=3$ level associated with the $D_{1}$ line at $795 \mathrm{~nm}$. The signal field is resonant with the $|b\rangle \leftrightarrow|c\rangle$ transition and the control field with the $|a\rangle \leftrightarrow|c\rangle$ transition.

When the signal field enters the atomic ensemble at Site $B$, its group velocity is strongly modified by the control field. By switching off the control field within about $100 \mathrm{~ns}$, the coupled excitation is converted into a spin wave excitation with a dominant dark state polariton component, i.e., the signal field is "stored" [42, 26, 27, 68]. An important condition to achieve this storage is a sufficiently large optical thickness of the atomic sample, which enables strong spatial compression of the incident signal field [22]. In our experiment the measured optical thickness $d \simeq 8$. The subsequent evolution of a dark state polariton in an external magnetic field is predicted to reveal a series of collapses and revivals whose structure is sensitive to the magnitude and orientation $\theta$ of the field relative to the signal wavevector [60].

\subsection{Theory}

As we deal with an unpolarized atomic ensemble, we must take into account the Zeeman degeneracy of the atomic levels. Choosing the same circular polarizations for both the probe and the control fields allows us to retain transparency [48]. For a $\sigma_{+}$polarized signal field, the dark state polariton annihilation operator for wavenumber $q$ is given by $[59,60]$

$$
\hat{\Psi}(q, t)=\frac{\Omega(t) \hat{a}_{k,+}-\sqrt{N p} g^{*} \sum_{m} R_{m} \hat{S}_{a m}^{b m}(q, t)}{\sqrt{|\Omega(t)|^{2}+N p|g|^{2} \sum_{m}\left|R_{m}\right|^{2}}}
$$

where $\Omega(t)$ is the control field Rabi frequency, $g$ the coupling coefficient for the signal

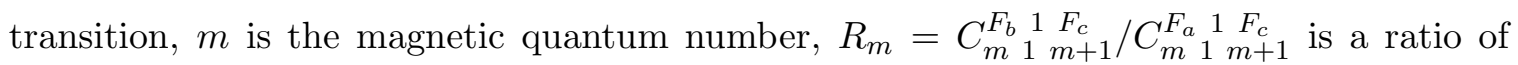
Clebsch-Gordan coefficients, $N$ is the number of atoms, $p=1 /\left(2 F_{b}+1\right), \hat{a}_{k,+}$ is the field annihilation operator for the mode of wavevector $k=q+\omega_{0} / c$ and positive helicity, $\omega_{0}$ is the Bohr frequency of the $|b\rangle \leftrightarrow|c\rangle$ transition, $S_{a m}^{b m}(q, t) \equiv 1 / \sqrt{N p} \sum_{\mu} \hat{\sigma}_{b m, a m}^{(\mu)}(t) \exp \left(-i\left(q z_{\mu}-\right.\right.$ $\left.\left.\Delta\left(t-z_{\mu} / c\right)\right)\right)$ is a collective spin wave operator, where $\hat{\sigma}_{b m, a m}^{(\mu)}(0)=|b, m\rangle_{\mu}\langle a, m|$ is a hyperfine coherence operator for atom $\mu=1, . . N, z_{\mu}$ is the position of atom $\mu$, and $\Delta$ is the hyperfine splitting of the ground state. When $R_{m}$ is finite for all $m$, the atomic 
configuration supports EIT, but when one or more $R_{m}$ is infinite, there is an unconnected lambda configuration, EIT is not possible and dark state polaritons do not exist. Specifically, the excited state $|c, m+1\rangle$ is not coupled by the control field to a state in the ground level $|a\rangle$. An atom in the state $|b, m\rangle$ would absorb the signal field as if no control field were present.

The signal is stored in the form of spin wave excitations associated with the dark state polaritons $\sim \sum_{m} R_{m} \hat{S}_{a m}^{b} m(q)$ for some range of $q$ 's. During the storage phase, and in the presence of the magnetic field $\mathbf{B}$, the atomic hyperfine coherences rotate according to the transformation

$$
\hat{S}_{a m}^{b m}(q, t)=\sum_{m_{1}=-F_{b}}^{F_{b}} \sum_{m_{2}=-F_{a}}^{F_{a}} \mathcal{D}_{m_{1} m}^{(b) \dagger}(t) \mathcal{D}_{m, m_{2}}^{(a)}(t) \hat{S}_{a m_{2}}^{b m_{1}}(q, 0),
$$

where $\mathcal{D}_{m, m^{\prime}}^{(s)}(t) \equiv\left\langle s, m\left|\exp \left(-i g_{s} \boldsymbol{\Omega} \cdot \hat{\mathbf{F}} t\right)\right| s, m^{\prime}\right\rangle$ is the rotation matrix element for hyperfine level $s, \hat{\mathbf{F}}$ is the total angular momentum operator, $\boldsymbol{\Omega} \equiv \mu_{B} \mathbf{B} / \hbar, \mu_{B}$ is the Bohr magneton, $g_{a}$ and $g_{b}$ are the Landé $g$ factors for levels $|a\rangle$ and $|b\rangle$ of ${ }^{85} \mathrm{Rb}$ and, ignoring the nuclear magnetic moment, $g_{a}=-g_{b}$. This rotation dynamically changes the dark state polariton population during storage.

The measured signal retrieved after a given storage time $T_{s}$ is determined by the remaining dark state polariton population. Stated differently, only the linear combination of hyperfine coherences $\sim \sum_{m} R_{m} \hat{S}_{a}^{b} \underset{m}{m}\left(q, T_{s}\right)$ contributes to the retrieved signal. We calculate the number of dark state polariton excitations as a function of $T_{s}$ using Eqs.(13) and (14), $\left\langle\hat{N}\left(T_{s}\right)\right\rangle=\sum_{q}\left\langle\hat{\Psi}^{\dagger}\left(q, T_{s}\right) \hat{\Psi}\left(q, T_{s}\right)\right\rangle$, and find

$$
\frac{\left\langle\hat{N}\left(T_{s}\right)\right\rangle}{\langle\hat{N}(0)\rangle}=\left|\sum_{m_{1} m_{2}} \frac{R_{m_{1}} R_{m_{2}}}{\sum_{m}\left|R_{m}\right|^{2}} \mathcal{D}_{m_{2} m_{1}}^{(b) \dagger}\left(T_{s}\right) \mathcal{D}_{m_{1} m_{2}}^{(a)}\left(T_{s}\right)\right|^{2}
$$

In Fig. 13, panels (f) through (j), we show the retrieval efficiency for various orientations of a magnetic field of magnitude $0.47 \mathrm{G}$, corresponding to the Larmor period of $4.6 \mu \mathrm{s}$. With the approximation $g_{a}=-g_{b}$ it is clear that the system undergoes a revival to the initial state after one Larmor period $\left(2 \pi /\left|g_{b} \boldsymbol{\Omega}\right|\right)$, and thus the signal retrieval efficiency equals the zero storage time value. Depending on the orientation of the magnetic field, a partial revival at half the Larmor period is also observed. For a magnetic field oriented along the 
$z$ axis (Fig. 13(f)), the polariton dynamics is relatively simple. Each hyperfine coherence $\hat{S}_{a}^{b} \underset{m}{m}$ merely picks up a phase factor that oscillates at frequency $2 m\left|g_{b} \boldsymbol{\Omega}\right|$, thus returning the system to its initial state at half the Larmor period. In this case, the partial revival is actually a full revival. On the other hand, for $\theta=\pi / 2$ (Fig. 13(j)), a rotation through half the Larmor period causes the coherence transformation $\hat{S}_{a}^{b} \underset{m}{m} \rightarrow-\hat{S}_{a-m}^{b-m}$, and as a result, the retrieval efficiency is reduced to $\left(\sum_{m} R_{m} R_{-m} / \sum_{m} R_{m}^{2}\right)^{2}$. The substructure within a half Larmor period is associated with interference of different hyperfine coherences contributing to the dark-state polariton [60].

To test these predictions, we apply a uniform dc magnetic field of magnitude $0.5 \pm 0.05 \mathrm{G}$ to the atomic ensemble using three pairs of Helmholtz coils. In our first set of measurements, $150 \mathrm{~ns}$ long coherent laser pulses containing on average $\simeq 5$ photons serve as the signal field. The outputs of the single-photon detectors D2 and D3 are fed into two "Stop" inputs of a time-interval analyzer which records the arrival times with a 2 ns time resolution. The electronic pulses from the detectors are gated for the period $\left[t_{0}, t_{0}+T_{g}\right]$, with $T_{g}=240$ ns, centered on the time determined by the control laser pulse during the retrieval stage. Counts recorded outside the gating period are therefore removed from the analysis. The recorded data allows us to determine the number of photoelectric events $N_{2}+N_{3}$, where $N_{i}$ is the total number of counts in the $i$-th channel $(i=1,2,3)$.

By measuring the retrieved field for different storage times and orientations of the magnetic field, we obtain the collapse and revival signals shown in Fig. 13, (a) through (e). As expected, we observe revivals at integer multiples of the Larmor period. In addition, we see partial revivals at odd multiples of half the Larmor period, except in the vicinity of $\theta=\pi / 4$. The measured substructures within a single revival period are in good agreement with the theory (cf., insets of Fig. 13, (e) and (j)). We attribute the overall damping of the revival signal in the experimental data to the magnetic field gradients. The evident decrease of this damping while $\theta$ is varied from 0 to $\pi / 2$ suggests that such gradients are predominantly along the direction of the signal field wavevector. Similarly, we attribute the additional broadening of the revival peaks at longer times to inhomogeneous magnetic 

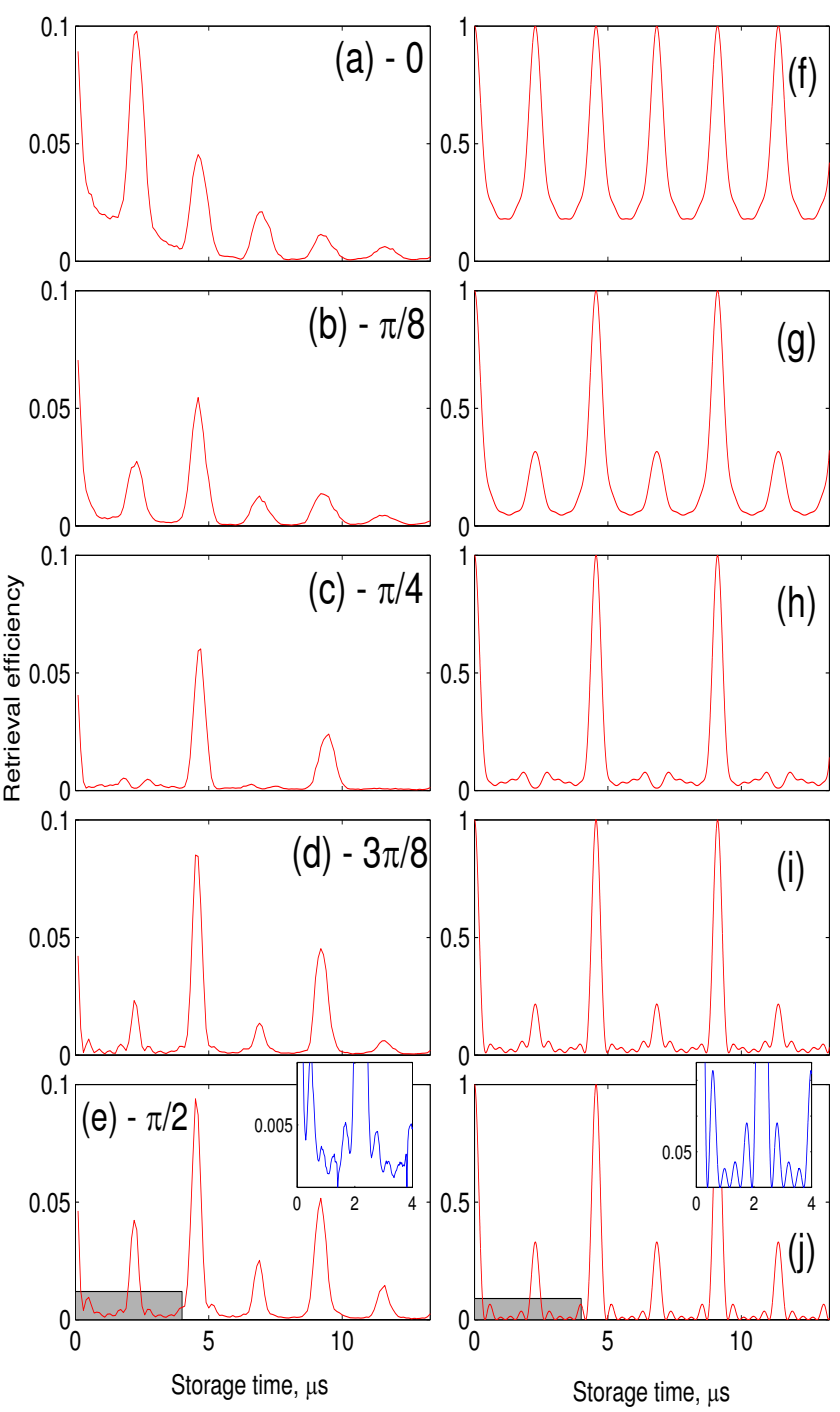

Figure 13: Panels (a)-(e) show the ratio of the number of photoelectric detection events for the retrieved and incident signal fields for various orientations, $\theta=0, \pi / 8, \pi / 4,3 \pi / 8, \pi / 2$, of the applied magnetic field, and as a function of storage time. The incident signal field is a weak coherent laser pulse. In all cases the control pulse is switched off at $T_{s}=0$. We observe a series of collapses and revivals at multiples of the half Larmor period of $2.3 \mu \mathrm{s}$. The observed damping over several Larmor periods is likely caused by residual magnetic field gradients. The inset in Panel (e) demonstrates the observed substructure within the first Larmor period. Panels (f) through (j) are corresponding theoretical plots of the dark-state polariton number calculated using Eq.(14). 


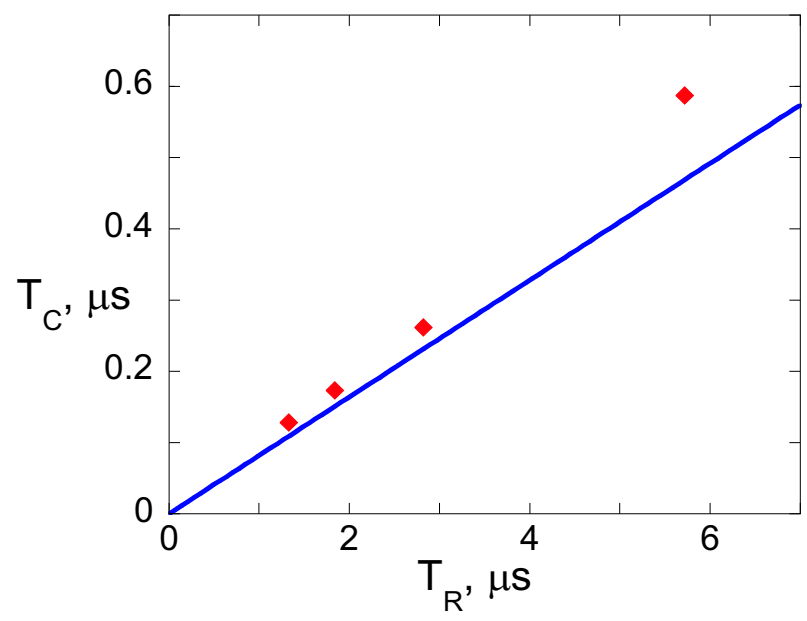

Figure 14: Diamonds show the measured collapse time $T_{C}$ of the first revival at half the Larmor period as a function of the measured revival time $T_{R}$, for magnetic field values of $0.8,0.6,0.4$, and $0.2 \mathrm{G}$, respectively, and for fixed orientation $\theta=\pi / 2$. The line shows the corresponding theoretical prediction $T_{C} \approx 0.082 T_{R}$ from Eq.(14).

fields, possibly ac fields, not included in the theoretical description. An additional investigations to determine the temporal and spatial characteristics of the residual magnetic fields are required. As an initial step Chapter 3 reports factor of three improvement in coherence time achieved due to better shielding of the ambient magnetic field.

Theory predicts that both the collapse and the revival times ( $T_{C}$ and $T_{R}$, respectively) scale inversely with the magnetic field [60]. In Fig. 14 the theoretical prediction $T_{C} \approx$ $0.082 T_{R}$ (solid line) is compared with the experimentally measured values. We find very good agreement except for the lowest value of magnetic field $B=0.2 \mathrm{G}$ which may be explained by the presence of residual magnetic field gradients.

In the measurements presented above, classical, coherent laser light was used as the signal field [30]. We have also investigated the revival dynamics of our atomic memory with the signal field in a single photon state, as shown in Fig. 12. The procedure for production of a single photon state of the signal field at Site $A$ is conditioned on the detection of an idler photon by D1 (see Refs.[47, 48] and Chapter 2 for further details). If photoelectric detections in different channels 1,2 or 1,3 happen within the same gating period, they contribute to the corresponding coincidence counts between $\mathrm{D} 1$ and $\mathrm{D} j, N_{1 j}, j=2,3$. We evaluate the $\alpha$ parameter of Grangier et al. [65], given by the ratio of various photoelectric 


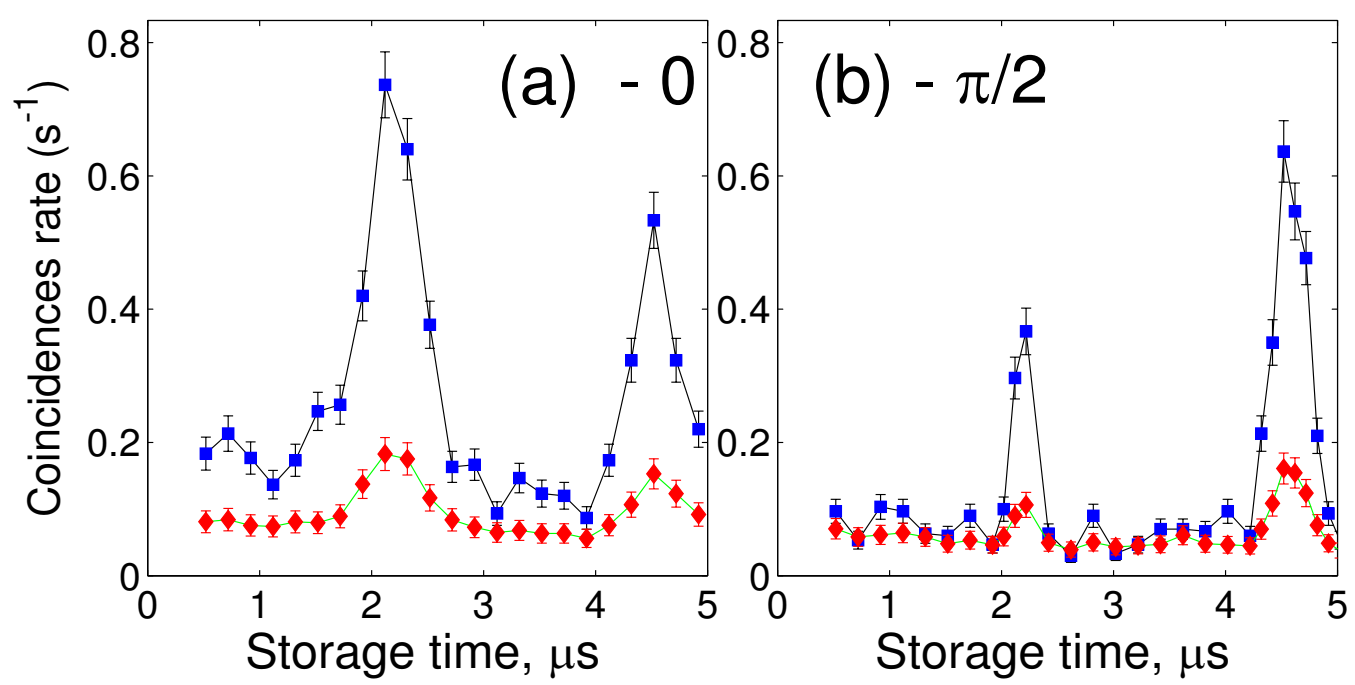

Figure 15: Squares show the measured rate of coincidence detections between D1 and D2,3, $N_{s i}=N_{12}+N_{13}$ as a function of the storage time. Diamonds show the measured level of random coincidences $N_{R}$. The ratio of squares to diamonds gives $g_{s i}$. Uncertainties are based on the statistics of the photoelectric counting events.

detection probabilities which are measured by the set of detectors D1,D2 and D3. For an ideal single-photon state $\alpha=0$, whereas for coherent fields $\alpha=1$. We have experimentally determined $\alpha=0.51 \pm 0.06$, without any correction for background or dark counts.

The normalized intensity cross-correlation function $g_{s i} \equiv\left(N_{12}+N_{13}\right) / N_{R}$ may be employed as a measure of non-classical signal-idler field correlations [30, 75], as discussed in detail in Ref.[43]. Here $N_{R} \equiv N_{1} \cdot\left(N_{2}+N_{3}\right) \cdot R_{\text {rep }}$ is the level of random coincidences, where $R_{\text {rep }}$ is the repetition rate of the experimental protocol. The values of $g_{s i}$ are obtained by the ratio of the upper and lower traces in Fig. 15. The measurements presented there give values of $g_{s i}$ well in excess of two at the revival times, suggesting the dark-state polaritons have a non-classical nature. One could further evaluate self-correlations for the idler field $g_{i i}$, and for the signal field $g_{s s}$, and confirm that the Cauchy-Schwarz inequality $g_{s i}^{2} \leq g_{s s} g_{i i}$ is indeed violated $[43,30,75]$. We have measured, by adding a beamsplitter and an additional detector, the value $g_{i i}=1.42 \pm 0.03$. When the signal field is stored and retrieved 500 ns later, we find that $g_{s s} \leq 2[48]$ (see also Chapter 2). While the total number of recorded coincidences between detectors D2 and D3 is not high enough to evaluate $g_{s s}$ for the revived 
polariton, it is also expected to be less than two, leading to a substantial violation of the Cauchy-Schwarz inequality. 


\section{CHAPTER IV}

\section{DETERMINISTIC SINGLE PHOTONS VIA CONDITIONAL QUANTUM EVOLUTION}

This chapter is based on Ref. [76].

\subsection{Motivation}

A seminal proposal to realize universal quantum computation using a single photon states of light and linear optics has been made by Knill, Laflamme and Milburn [61]. A significant step in the direction of quantum networks is the capability to generate deterministic single photons. Previous implementations of deterministic single photon sources used single emitters, such as quantum dots $[77,78,79]$, color centers [80, 81], neutral atoms [20, 82], ions [83], and molecules [84].

By contrast, in this Chapter we describe combination of an intrinsically probabilistic heralded single photon source, based on an ensemble of about one million atoms, with measurement and conditional quantum evolution, to generate single photons on demand. A heralded source of single photons involves the generation of photon pairs, in which the photoelectric detection of one of the pair signals the presence of the other, idler photon. The heralding event is inherently random, and the unconditioned field state consists mostly of vacuum $[85,65]$. Hence, heralded single photon sources are characterized by mean photon number $\langle\hat{n}\rangle \ll 1$. Without exception all prior experiments with atomic ensembles could only function as heralded single photon sources [46, 47, 49, 48, 60, 23, 86, 52, 54, 55].

\subsection{Protocol for deterministic single photon generation}

Our deterministic source amplifies the single photon component of a heralded source at the expense of the vacuum through the use of atomic memory and quantum measurementbased feedback. In earlier work quantum feedback protocols have demonstrated control of 


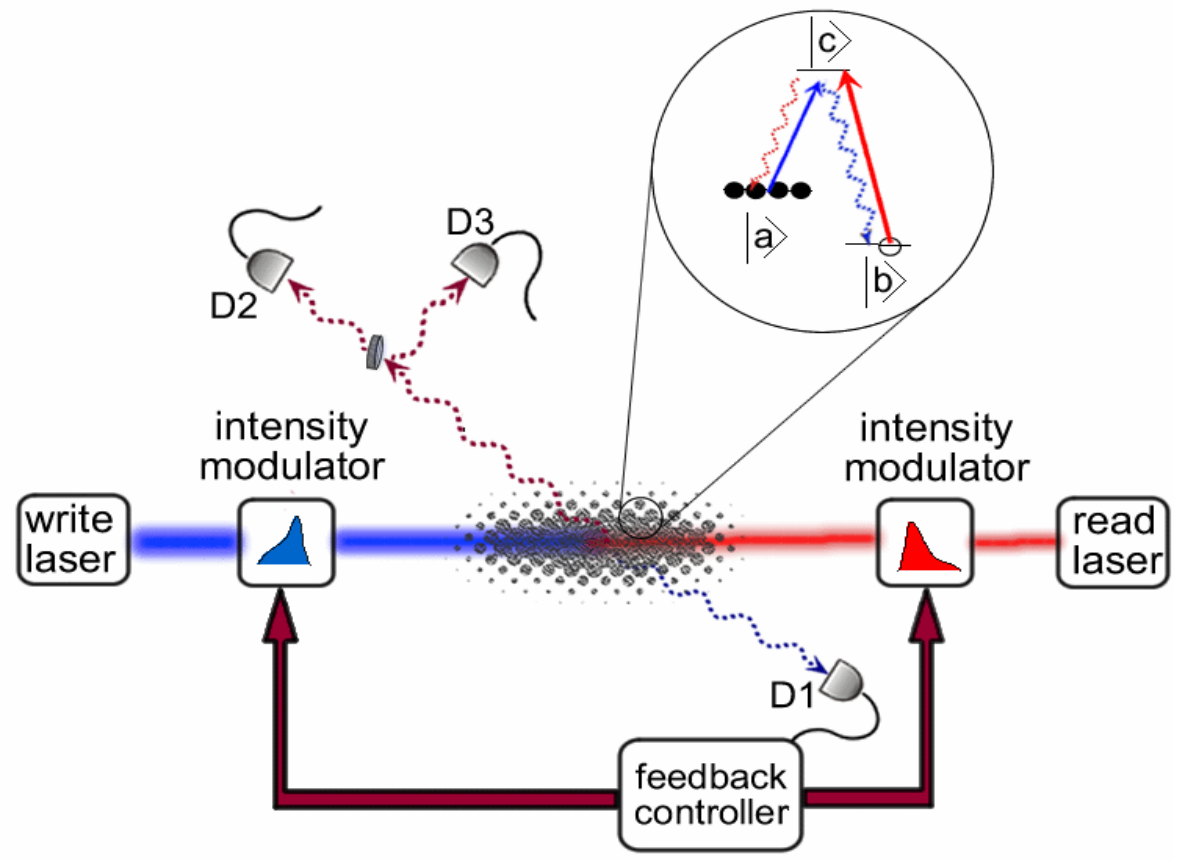

Figure 16: Schematic of experimental setup for deterministic single photon generation, with the inset showing the three-level atomic configuration involved (see text for details).

non-classical states of light [87] and motion of a single atom [88] in cavity QED. We first outline the procedure for heralded single photon generation. A schematic of our experiment is shown in Fig. 16. An optically thick atomic cloud is provided by a magneto-optical trap (MOT) of ${ }^{85} \mathrm{Rb}$. The ground levels $\{|a\rangle ;|b\rangle\}$ correspond to the $5 S_{1 / 2}, F_{a, b}=\{3,2\}$ hyperfine levels, while the excited level $|c\rangle$ represents the $\left\{5 P_{1 / 2}, F_{c}=3\right\}$ level of the $D_{1}$ line at $795 \mathrm{~nm}$. The experimental sequence starts with all of the atoms prepared in level $|a\rangle$. An amplitude modulator generates a linearly polarized $70 \mathrm{~ns}$ long write pulse tuned to the $|a\rangle \rightarrow|c\rangle$ transition, and focused into the MOT with a Gaussian waist of about $430 \mu \mathrm{m}$. We describe the write process using a simple model based on nondegenerate parametric amplification. The light induces spontaneous Raman scattering via the $|c\rangle \rightarrow|b\rangle$ transition. The annihilation of a write photon creates a pair of excitations: namely a signal photon and a quasi-bosonic collective atomic excitation [11]. The scattered light with polarization orthogonal to the write pulse is collected by a single mode fiber and directed onto a single photon detector $\mathrm{D} 1$, with overall propagation and detection efficiency $\eta_{s}$. The state of the atomic excitation conditioned on this photoelectric event depends, in general, on the 
detection time. However, in the limit of long detector dead time and when the time of detection is not recorded, the conditioned density operator for the atomic excitation $A$ is given by

$$
\rho_{A \mid 1}=\frac{1}{p_{1}} \sum_{n=1}^{\infty} \frac{\tanh ^{2 n} \chi}{\cosh ^{2} \chi}\left(1-\left(1-\eta_{s}\right)^{n}\right)|n\rangle\langle n|,
$$

where $p_{1} \ll 1$ is the probability of a signal photoelectric detection event per write pulse and the interaction parameter $\chi$ is given in terms of $p_{1}$ and $\eta_{s}$ by

$$
\sinh ^{2} \chi=p_{1} /\left[\eta_{s}\left(1-p_{1}\right)\right]
$$

where $|n\rangle \equiv \hat{A}^{\dagger n}|0\rangle / \sqrt{n !}$, and $|0\rangle$ is the atomic vacuum. We note that in Eq. (16) there is zero probability to find $|0\rangle$.

After a storage time $\tau$, a read pulse of length $80 \mathrm{~ns}$ containing around $3 \cdot 10^{7}$ photons, and with polarization orthogonal to that of the write pulse, tuned to the $|b\rangle \rightarrow|c\rangle$ transition, illuminates the atomic ensemble (Fig. 16). Ideally, the read pulse converts atomic spin excitations into the idler field emitted on the $|c\rangle \rightarrow|a\rangle$ transition. The elastically scattered light from the write beam is filtered out, while the idler field polarization orthogonal to that of the read beam is directed into a 50:50 single-mode fiber beamsplitter. Both write/read and signal/idler pairs of fields are counter-propagating [54]. The waist of the signal-idler mode in the MOT is about $180 \mu \mathrm{m}$. The two outputs of the fiber beamsplitter are connected to detectors D2 and D3. Electronic pulses from the detectors are gated with $120 \mathrm{~ns}$ (D1) and 100 ns (D2 and D3) windows centered on times determined by the write and read light pulses, respectively. Subsequently, the electronic pulses from D1, D2, and D3 are fed into a time-interval analyzer which records photoelectric detection events with a 2 ns time resolution.

The transfer of atomic excitation to the idler field and subsequent detection can be modeled by a linear optics relation $\hat{a}_{i}(\tau)=\sqrt{\epsilon_{i}(\tau)} \hat{A}+\sqrt{1-\epsilon_{i}(\tau)} \hat{\xi}(\tau)$, where $\hat{a}_{i}$ is the idler annihilation operator for the detected field mode, and $\hat{\xi}(\tau)$ is a bosonic operator which accounts for coupling to degrees of freedom other than the detected idler mode. The efficiency $\eta_{i}(\tau)$ is the probability that a single atomic excitation stored for $\tau$ results in a photoelectric event at either $D 2$ or $D 3$, and includes the effects of idler retrieval 
and propagation losses and non-unit detector efficiency. Using multimode detection theory we calculate the probabilities $p_{k \mid 1}(\tau)$ that detector $D k(k=2,3)$ registers a photoelectric detection event, within the entire idler field envelope, conditioned on the signal detection event at D1. We also calculate the conditional probability $p_{23 \mid 1}(\tau)$ of a photoelectric event occurring at both detectors. These probabilities are given by

$$
\begin{aligned}
p_{2 \mid 1}(\tau) & =p_{3 \mid 1}(\tau)=\Pi\left(\eta_{i}(\tau) / 2 ; p_{1}, \eta_{s}\right) \\
p_{23 \mid 1}(\tau) & =p_{2 \mid 1}(\tau)+p_{3 \mid 1}(\tau)-\Pi\left(\eta_{i}(\tau) ; p_{1}, \eta_{s}\right)
\end{aligned}
$$

where

$$
\frac{1}{p_{1}}\left(\frac{1}{1+\eta \sinh ^{2} \chi}-\frac{\Pi\left(\eta ; p_{1}, \eta_{s}\right)=1-}{1+\left(\eta_{s}+\eta\left(1-\eta_{s}\right)\right) \sinh ^{2} \chi}\right)
$$

is the probability that a detector registers a photoelectric event conditioned on a signal detection.

The protocol for our deterministic single photon source has two crucial requirements: high-efficiency heralded single photon generation and long atomic memory times. Earlier experiments demonstrated proof-of-principle heralded single photon generation [43, 45, 44, 46]. However, the measured efficiencies and memory times were much too low to provide the basis for a deterministic source based on our protocol. Improved efficiencies and longer atomic memory times were subsequently reported in Refs. [47, 48, 89], but these were still insufficient.

\subsection{Results}

In Figs. 17 and 18 we show the results of our characterization of an improved source of heralded single photons. Panel (a) of Fig. 17 shows the measured intensity cross-correlation

function $g_{s i} \equiv\left[p_{2 \mid 1}+p_{3 \mid 1}\right] /\left[p_{2}+p_{3}\right]$ as a function of $p_{1}$. Large values of $g_{s i}$ under conditions of weak excitation - i.e., small $p_{1}$ - indicate strong pairwise correlations between signal and idler photons. The efficiency of the signal photon generation and detection is given by $\eta_{s} \rightarrow g_{s i} p_{1}$, in the limit $\sinh ^{2} \chi \ll 1$. We have measured $\eta_{s} \approx 0.08$, which includes the effects 
of passive propagation and detection losses $\epsilon_{s}$. It is important to distinguish the measured efficiency from the intrinsic efficiency which is sometimes employed. The intrinsic efficiency of having a signal photon in a single spatial mode at the input of the single-mode optical fiber $\eta_{s}^{0} \equiv\left(\eta_{s} / \epsilon_{s}\right) \approx 0.24$. We measure $\epsilon_{s} \equiv \epsilon_{s}^{f} \epsilon_{s}^{t} \epsilon_{s}^{d} \approx 0.3$ independently using coherent laser light, where the fiber coupling efficiency $\epsilon_{s}^{f} \approx 0.7$, optical elements transmission $\epsilon_{s}^{t} \approx 0.85$, and the detection efficiency $\epsilon_{s}^{d} \approx 0.55$. The measured efficiency of the idler photon detection is $\eta_{i} \rightarrow g_{s i}\left(p_{2}+p_{3}\right) \approx 0.075$. Here $p_{2}$ and $p_{3}$ are defined by expressions analogous to Eq. (17). Similarly, the intrinsic efficiency for the idler field $\eta_{i}^{0} \equiv\left(\eta_{i} / \epsilon_{i}\right) \approx 0.34$, where we measure $\epsilon_{i} \equiv \epsilon_{i}^{f} \epsilon_{i}^{t} \epsilon_{i}^{d} \approx 0.22$, with $\epsilon_{i}^{f} \approx 0.75, \epsilon_{i}^{t} \approx 0.59$, and $\epsilon_{i}^{d} \approx 0.55$. The reported values of $\eta_{s} \approx 0.08$ and $\eta_{i} \approx 0.075$ represent slight improvements on the previous highest measured efficiencies in atomic ensemble experiments of $0.04-0.07[48,49]$ (see also Chapters 2 and $3)$.

The quality of the heralded single photons produced by our source is assessed using the procedure of Grangier et al., which involves a beamsplitter followed by two single photon counters, as shown in Fig. 16 [65]. An ideal single-photon input to the beamsplitter results in photoelectric detection at either D2 or D3, but not both. An imperfect single photon input will result in strong anticorrelation of the coincidence counts. Quantitatively, this is determined by the anticorrelation parameter $\alpha$ given by the ratio of various photoelectric detection probabilities measured by the set of detectors D1,D2 and D3: $\alpha=p_{23 \mid 1} /\left(p_{2 \mid 1} p_{3 \mid 1}\right)$. Classical fields must satisfy a criterion $\alpha \geq 1$ based on the Cauchy-Schwarz inequality [65]. For an ideally prepared single photon state $\alpha \rightarrow 0$. Panel (b) shows the measured values of $\alpha$ as a function of $p_{1}$, with $\min \{\alpha\}=0.012 \pm 0.007$ representing a ten-fold improvement on the lowest previously reported value in atomic ensembles [48].

In order to evaluate the atomic memory coherence time $\tau_{c}$, we measure $g_{s i}$ as a function of the storage time $\tau$, Fig. 18. To maximize $\tau_{c}$, the quadrupole coils of the MOT are switched off, with the ambient magnetic field compensated by three pairs of Helmholtz coils [47]. The measured value of $\tau_{c} \approx 31.5 \mu \mathrm{s}$, a three-fold improvement over the previously reported value, is limited by dephasing of different Zeeman components in the residual magnetic field $[48,49]$. 

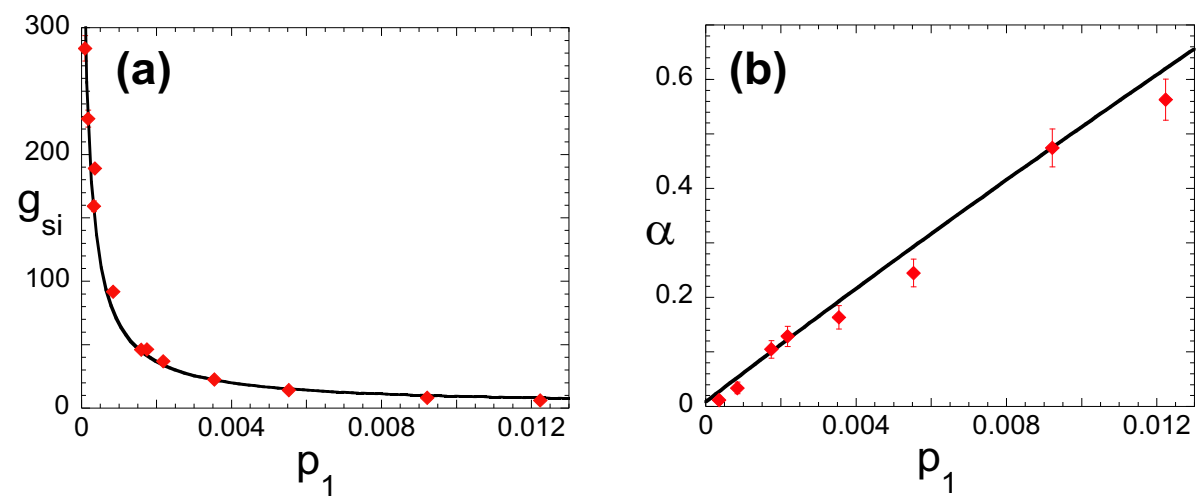

Figure 17: Correlation functions $g_{s i}$ (panel (a)) and $\alpha$ (panel (b)) as a function of $p_{1}$, taken at $\tau=80 \mathrm{~ns}$. The solid lines are based on Eqs. $(18,19)$, with addition of a nearly-negligible background contribution.

The long coherence time enables us to implement a conditional quantum evolution protocol. In order to generate a single photon at a predetermined time $t_{p}$, we initiate the first of a series of trials at a time $t_{p}-\Delta t$, where $\Delta t$ is on the order of the atomic coherence time $\tau_{c}$. Each trial begins with a write pulse. If D1 registers a signal photoelectric event, the protocol is halted. The atomic memory is now armed with an excitation and is left undisturbed until the time $t_{p}$ when a read pulse converts it into the idler field. If D1 does not register an event, the atomic memory is reset to its initial state with a cleaning pulse, and the trial is repeated. The duration of a single trial $t_{0}=300 \mathrm{~ns}$. Even if D1 does not register a photoelectric event after repeated trials, the protocol is halted $1.5 \mu$ s prior to $t_{p}$, and the idler is detected.

This experimental sequence effectively amplifies the single photon component at the expense of the vacuum. Armed with Eqs. (18) and (19), we can calculate the unconditioned detection and coincidence probabilities for the complete protocol. The probability that the atomic excitation is produced on the $k^{t h}$ trial is $p_{1}\left(1-p_{1}\right)^{k-1}$. This excitation is stored for a time $(N-k) t_{0}$ before it is retrieved and detected, $N=\delta t / t_{0}$ is the maximum number of trials that can be performed in the protocol (we ignore the $1.5 \mu$ s halting period before the read-out).

One can express the probability of a photoelectric event at $D i(i=2,3), P_{i}$, and the 


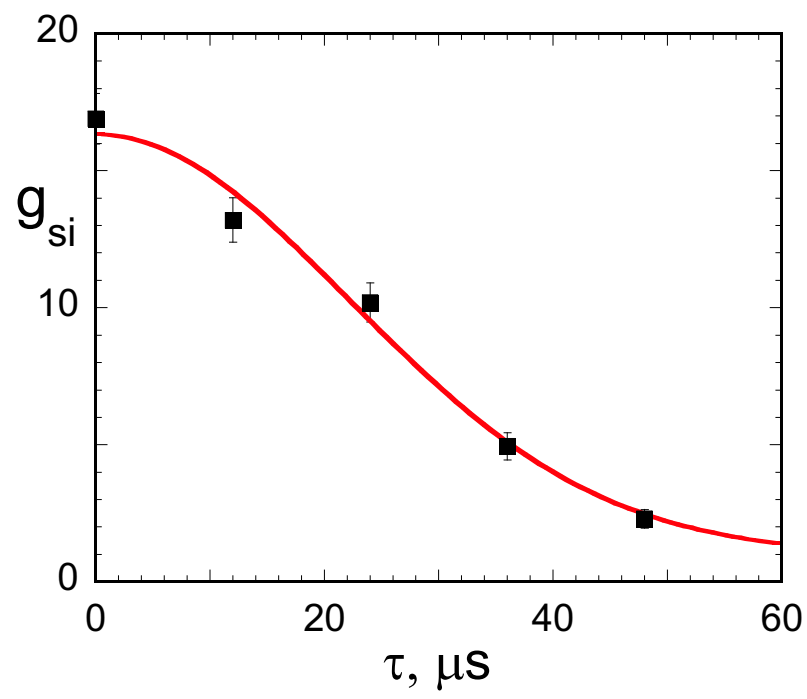

Figure 18: Normalized signal-idler intensity correlation function $g_{s i}$ as a function of the storage time $\tau$. The full curve is a fit of the form $1+B \exp \left(-\tau^{2} / \tau_{c}^{2}\right)$ with $B=16$ and the collapse time $\tau_{c}=31.5 \mu \mathrm{s}$ as adjustable parameters.

coincidence probabilities $P_{23}$ in terms of the conditional probabilities of Eqs. (18) and (19),

$$
\begin{aligned}
P_{i} & =p_{1} \sum_{k=1}^{N}\left(1-p_{1}\right)^{k-1} p_{i \mid 1}\left(t_{p}-k t_{0}\right), \\
P_{23} & =p_{1} \sum_{k=1}^{N}\left(1-p_{1}\right)^{k-1} p_{23 \mid 1}\left(t_{p}-k t_{0}\right) .
\end{aligned}
$$

In the limit of infinite atomic coherence time and $N \rightarrow \infty, P_{i} \rightarrow p_{i \mid 1}$, and $P_{23} \rightarrow p_{23 \mid 1}$. Hence, if the memory time is sufficiently long for an adequate number of trials, the protocol ideally results in deterministic preparation of a single atomic excitation, which can be converted into a single photon at a desired time. Consistent with Fig.18 we assume a combined retrieval-detection efficiency that decays as a Gaussian function of storage time, $\eta_{i}(\tau)=\eta_{i}(0) e^{-\left(\tau / \tau_{c}\right)^{2}}$, where $\tau_{c}$ is the atomic spin-wave coherence time. We emphasize that in the absence of information about the signal field the reduced density operator of the idler is thermal [75]. In contrast, the evolution of the idler conditioned by the recorded measurement history of the signal field in our protocol, ideally results in a single photon state.

In Fig. 19 we present the measured degree of 2 nd order coherence for zero time delay $g_{D}^{(2)}(0) \equiv P_{23} /\left(P_{2} P_{3}\right)[90]$ and the measured efficiency $\eta_{D} \equiv P_{2}+P_{3}$ as a function of $N$ (panels (a) and (b)), and as a function of $p_{1}$ (panels (c) and (d)). The solid curves are 

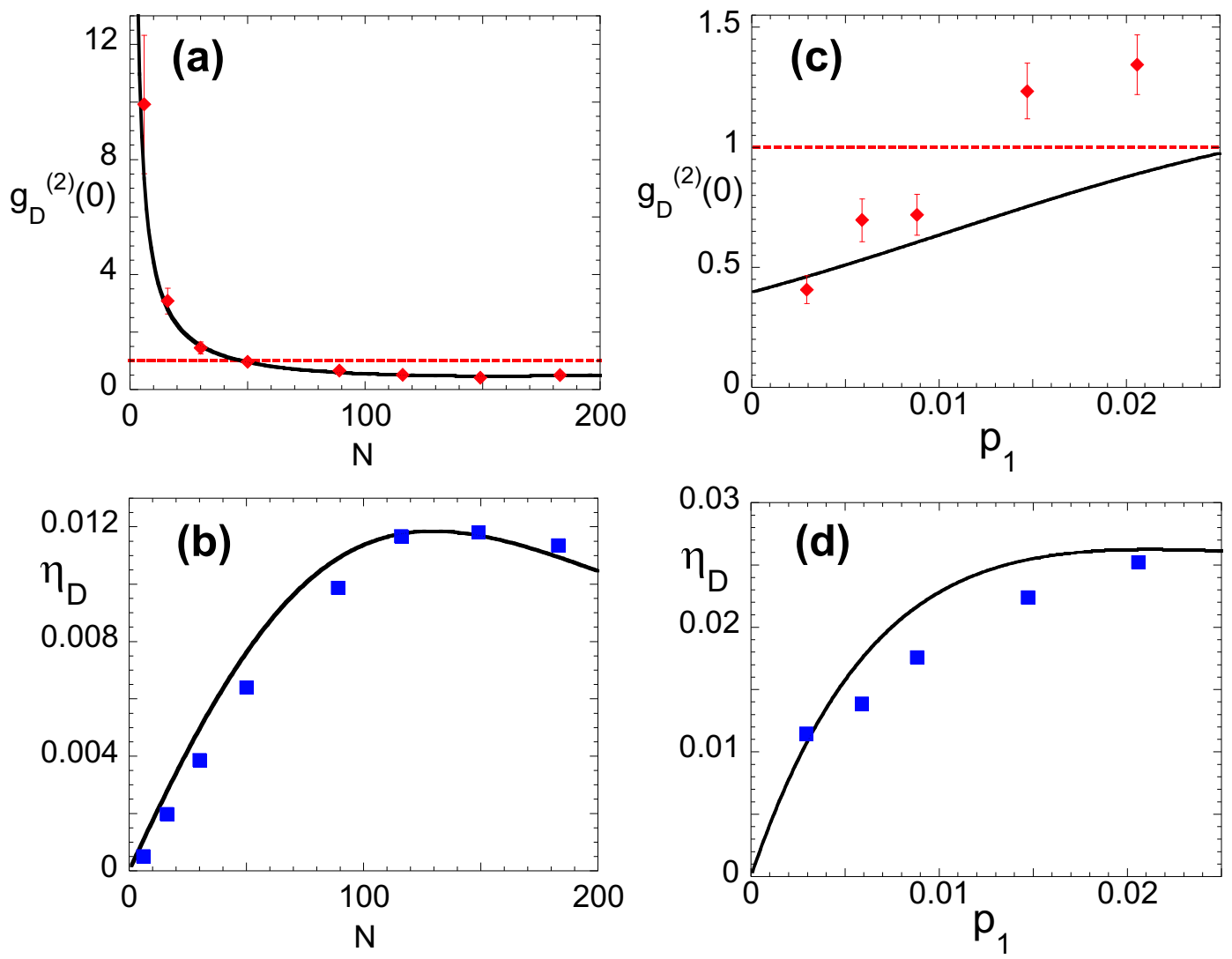

Figure 19: $g_{D}^{(2)}(0)$ as a function of maximum number of trials $N$ (panel (a)) and $p_{1}$ (panel (c)); measured efficiency to generate and detect a single photon $\eta_{D}$ as a function of $N$ (panel (b)) and $p_{1}$ (panel (d)). For panels (a) and (b) $p_{1}=0.003$, whereas for for panels (c) and (d) $N=150$. The full curves are based on Eqs. (21) and (22) with the values of efficiencies and coherence times given in the text, with however $\eta_{D}$ multiplied by an empirical factor of $2 / 3$. We believe this reduced efficiency is due to imperfect switching of the read light in the feedback-based protocol (we note that there are no other adjustable parameters in the simple theory presented). Evident deviations from the theory in panels (c) and (d), beyond the statistical uncertainties associated with photoelectric counting events, could be explained either by inadequacies of the theory, or slow systematic drifts in the residual magnetic field and the read light leakage. 
based on Eqs.(21) and Eqs.(22) above. The dashed lines in panels (a) and (c) show the expected value of $g_{D}^{(2)}(0)=1$ for a weak coherent state (as we have confirmed in separate measurements). The particular value of $\Delta t$ is chosen to optimize $g_{D}^{(2)}(0)$ and $\eta_{D}$. The minimum value of $g_{D}^{(2)}(0)=0.41 \pm 0.04$ indicates substantial suppression of two-photon events and under the same conditions $\eta_{D}=0.012$. The corresponding value of the measured Mandel parameter $Q_{D} \equiv-\eta_{D}\left(1-g_{D}^{(2)}(0)\right)$ is $\approx-0.007 \pm 10 \%$ and is largely determined by $\eta_{D}[90]$. Note that in the limit of infinite atomic memory and $N \rightarrow \infty, g_{D}^{(2)}(0) \rightarrow \min \{\alpha\} \approx$ $0.012 \pm 0.007$ and $\eta_{D} \rightarrow \eta_{i} \approx 0.075$. We note that this light source is stationary, with the sub-Poissonian character of photoelectric events unaffected by probabilistic atom loading, a randomness inherent to a cavity QED source [20, 21].

Moreover, the measured efficiency $\eta_{D}$ can be further increased by employing atomic sample with larger optical thickness and by optimizing the spatial focusing patterns of the signal and idler fields. (In separate sets of measurements, we have observed $\eta_{s} \approx 0.2$, for the intrinsic signal efficiency $\left.\eta_{s}^{0} \approx 0.6\right)$. In principle, the spatial signal-idler correlations from an atomic ensemble (and, therefore $\eta_{i}^{0}$ ) can also be improved by use of an optical cavity. However, in the absence of special precautions the use of a cavity will itself introduce additional losses associated, e.g., with the mirror coatings or the cavity locking optics [20, $21,83,55]$. The measured efficiency $\eta_{D}$ would involve a trade-off between improved spatial correlations due to the cavity and the concomitant losses that it introduces. 


\section{CHAPTER V}

\section{QUANTUM STATE TRANSFER BETWEEN MATTER AND LIGHT}

This chapter is based on Ref. [46].

\subsection{Theory}

The ability to coherently transfer quantum information between photonic- and materialbased quantum systems is a prerequisite for all practical distributed quantum computation and scalable quantum communication protocols [1].

Here we report on the experimental realization of coherent quantum state transfer from a matter qubit onto a photonic qubit, utilizing an optically thick cold atomic cloud. Our experiment involves three steps:

1. An entangled state between a single photon (signal) and a single collective excitation distributed over many atoms in two distinct optically thick atomic samples is generated.

2. Measurement of the signal photon projects the atomic ensembles into a desired state, conditioned on the choice of the basis and the outcome of the measurement. Ideally this atomic state is a nearly maximally entangled state between two distinct atomic ensembles.

3. This state is converted into a single photon (idler) emitted into a well-defined mode, without using a high-finesse cavity. These three ingredients constitute a complete set of tools required to build an arbitrary large-scale quantum network [11].

As illustrated in Fig.20A, the classical laser pulses used in the generation and verification procedures define the two distinct pencil-shape components of the atomic ensemble that 
form our memory qubit, L and R. Fig.20B indicates schematically the structure of the four atomic levels involved, $|a\rangle,|b\rangle,|c\rangle$ and $|d\rangle$. The experimental sequence starts with all of the atoms prepared in state $|a\rangle$. A write pulse tuned to the $|a\rangle \rightarrow|c\rangle$ transition is split into two beams by a polarizing beam splitter (PBS1) and passed through the atomic sample. The light induces spontaneous Raman scattering on the $|c\rangle \rightarrow|b\rangle$ transition. The classical write pulse is so weak that less than one photon is scattered in this manner into the forward direction mode for each pulse in either $\mathrm{L}$ or $\mathrm{R}$. The forward scattered mode is dominantly correlated with a distinct collective atomic state [11]. In the first order of perturbation theory in the atom-light coupling $\chi$, the atom-light state is

$$
|\Psi\rangle \sim|a\rangle_{1} \ldots|a\rangle_{N_{L}+N_{R}}\left|0_{p}\right\rangle_{L}\left|0_{p}\right\rangle_{R}+\chi\left(\left|L_{a}\right\rangle\left|1_{p}\right\rangle_{L}\left|0_{p}\right\rangle_{R}+\left|R_{a}\right\rangle\left|0_{p}\right\rangle_{L}\left|1_{p}\right\rangle_{R}\right)
$$

We have defined two effective states of the atomic ensembles:

$$
\begin{array}{r}
\left|L_{a}\right\rangle=\sum_{i=1}^{N_{L}} g_{i}|a\rangle_{1} \ldots|b\rangle_{i} \ldots|a\rangle_{N_{L}} \ldots|a\rangle_{N_{L}+N_{R}} \\
\left|R_{a}\right\rangle=\sum_{j=N_{L}+1}^{N_{L}+N_{R}} g_{j}|a\rangle_{1} \ldots|a\rangle_{N_{L}} \ldots|b\rangle_{j} \ldots|a\rangle_{N_{L}+N_{R}},
\end{array}
$$

with the weights $g_{i}, g_{j}$ determined by the write field intensity distribution, $\sum_{i=1}^{N_{L}}\left|g_{i}\right|^{2}=1$, $\sum_{j=N_{L}+1}^{N_{L}+N_{R}}\left|g_{j}\right|^{2}=1[91,63] .\left|L_{a}\right\rangle$ and $\left|R_{a}\right\rangle$ have properties of a two-level system (qubit): $\left\langle L_{a} \mid L_{a}\right\rangle=1,\left\langle R_{a} \mid R_{a}\right\rangle=1,\left\langle L_{a} \mid R_{a}\right\rangle=0$. Although the interaction of the light with the atoms is non-symmetric with respect to permutation of atoms, the second term in Eq.23 in fact describes a strongly entangled atom-photon state in the sense of [63]. Using PBS4 and a half wave plate inserted into one of the channels, we map the two spatial modes associated with the two ensembles into a single spatial mode with polarization encoding of the light's origin: $\left|1_{p}\right\rangle_{L} \rightarrow|H\rangle_{s} ;\left|1_{p}\right\rangle_{R} \rightarrow|V\rangle_{s}$, where $H$ and $V$ indicate horizontal and vertical polarization, respectively, and $s$ denotes signal. Next, the light is passed through an arbitrary polarization state transformer $R_{s}\left(\theta_{s}, \phi_{s}\right)$ and a polarizer PBS5, so that the state at the output of PBS5 is

$$
\left|H^{\prime}\right\rangle=\cos \left(\theta_{s}\right) e^{i \phi_{s}}|H\rangle_{s}+\sin \left(\theta_{s}\right)|V\rangle_{s},
$$

and is directed onto a single-photon detector D1. When D1 detects a photon, the joint 
state in Eq.23 is projected into the desired atomic state

$$
\left|\Psi_{a}\right\rangle=\cos \left(\theta_{s}\right) e^{-i \phi_{s}}\left|L_{a}\right\rangle+\sin \left(\theta_{s}\right) e^{i \eta_{s}}\left|R_{a}\right\rangle
$$

which is an entangled state of the two atomic samples $L$ and $R$. Phase $\eta_{s}$ is determined by the difference in length of the two paths $L$ and $R$. After a variable delay time $\Delta t$ we convert the atomic excitation into a single photon by illuminating the atomic ensemble with a pulse of light near resonant with the $|b\rangle \rightarrow|d\rangle$ transition. For an optically thick atomic sample, the photon will be emitted with high probability into the spatial mode determined by the write pulse $[11,91]$ achieving memory read-out:

$$
\left|\Psi_{a}\right\rangle=\cos \left(\theta_{s}\right) e^{-i \phi_{s}}\left|L_{a}\right\rangle+\sin \left(\theta_{s}\right) e^{i \eta_{s}}\left|R_{a}\right\rangle \rightarrow|\Psi\rangle_{i}=\cos \left(\theta_{s}\right) e^{-i \phi_{s}}|H\rangle_{i}+\sin \left(\theta_{s}\right) e^{i\left(\eta_{i}+\eta_{s}\right)}|V\rangle_{i} .
$$

That is, the polarization state of the idler photon $i$ is uniquely determined by the observed state of the signal photon. Alternatively, one could store the signal in a fiber until after the read-out. In that case, the two-photon signal-idler state would ideally be an entangled state:

$$
\left|\Psi_{M}\right\rangle=|v a c\rangle+\chi \frac{1}{\sqrt{2}}\left(|H\rangle_{s}|H\rangle_{i}+e^{i\left(\eta_{s}+\eta_{i}\right)}|V\rangle_{s}|V\rangle_{i}\right)
$$

\subsection{Experiment}

A magneto-optical trap (MOT) of ${ }^{85} \mathrm{Rb}$ is used to provide an optically thick atomic cloud for our experiment (See Fig. 20). The ground states $\{|a\rangle ;|b\rangle\}$ correspond to the $5 S_{1 / 2}, F=$ $\{3,2\}$ levels of ${ }^{85} \mathrm{Rb}$, while the excited states $\{|c\rangle ;|d\rangle\}$ represent the $\left\{5 P_{3 / 2}, F=3 ; 5 P_{1 / 2}, F=\right.$ $2\}$ levels of the $\left\{D_{2}, D_{1}\right\}$ lines at $\{780 ; 795\} \mathrm{nm}$, respectively. The experimental sequence starts with all of the atoms prepared in state $|a\rangle$ via optical pumping, after shutting off the trapping and cooling light.

A $140 \mathrm{~ns}$ long write pulse tuned to the $|a\rangle \rightarrow|c\rangle$ transition is split into two beams by a polarizing beamsplitter PBS1 and focused into two regions of the MOT about $1 \mathrm{~mm}$ apart with Gaussian waists of about $50 \mu \mathrm{m}$. PBS2 and PBS3 separate the horizontally polarized component of the forward scattered light from the vertically polarized classical pulse. After being mixed by PBS4, the light goes through the quarter- and the half-wave plates that 


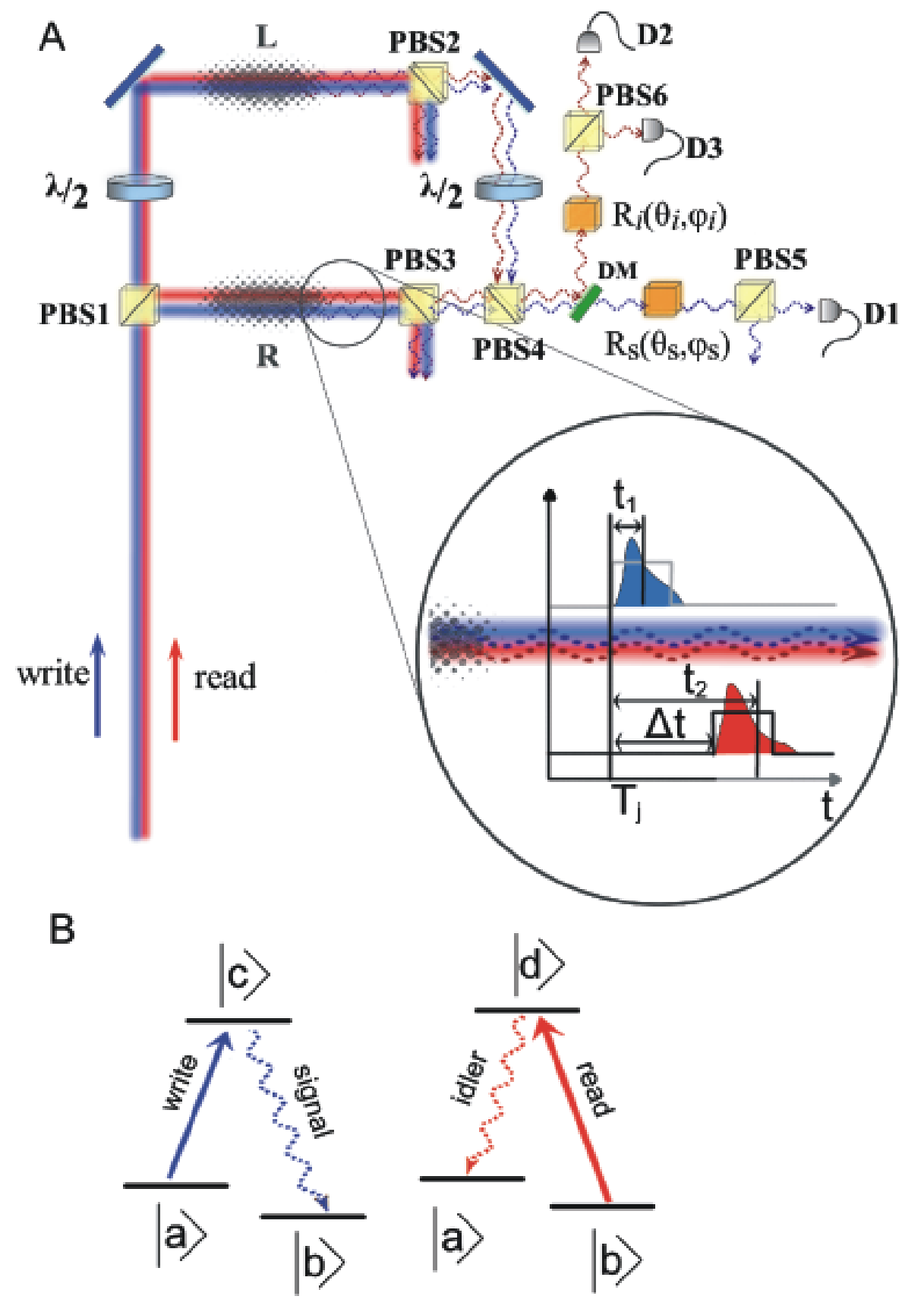

Figure 20: (A) Schematic of experimental setup. PBS1-6, polarizing beam splitters, $\lambda / 2$, half waveplate, polarization state transformers, $R_{s}\left(\theta_{s}, \phi_{s}\right)$ and $R_{i}\left(\theta_{i}, \phi_{i}\right)$, (D1,D2,D3), single photon detectors, DM, dichroic mirror. The inset illustrates the timing of the write and read pulses. (B) The relevant atomic level structure. 
provide the state transformation $R_{s}\left(\theta_{s}, \phi_{s}\right)$. The light continues to another polarizer PBS5, and is directed to a single photon detector D1. Detection of one photon by D1 prepares the atomic ensemble in any desired state in the basis of $\left|L_{a}\right\rangle,\left|R_{a}\right\rangle$ determined by $R_{s}\left(\theta_{s}, \phi_{s}\right)$ and thereby concludes the preparation of the quantum memory qubit.

Following memory state preparation, the read-out stage is performed. After a userprogrammable delay $\Delta t$, a 115 ns long read pulse tuned to the $|b\rangle \rightarrow|d\rangle$ transition illuminates the two atomic ensembles. This accomplishes a transfer of the memory state onto the single photon (idler) emitted by the $|d\rangle \rightarrow|a\rangle$ transition. After passing through the state transformer $R_{i}\left(\theta_{i}, \phi_{i}\right)$ and PBS6, the two polarization components are directed onto single-photon detectors (D2, D3) thus accomplishing measurement of the idler photon, and hence the memory qubit, in a controllable arbitrary basis.

As in any real experiment, various imperfections prevent the read-out of the quantum memory (idler photon) from being identical to the state that we intended to write into the memory. To quantify the degree to which we faithfully prepare and read-out the quantum memory, we measure the polarization correlations between the signal and idler photons. The observed correlations allow us to characterize the extent to which our procedures are working. To investigate the storage capabilities of our memory qubit quantitatively, we use time-resolved detection of the signal and idler photons for two values of delay $\Delta t$ between the application of the write and read pulses, $100 \mathrm{~ns}$ and $200 \mathrm{~ns}$. The electronic pulses from the detectors are gated with $250 \mathrm{~ns}$ and $140 \mathrm{~ns}$ windows centered on the time determined by the write and read light pulses, respectively. Afterwards, the electronic pulses are fed into a time-interval analyzer (with $\delta=2$ ns time resolution). In order to measure the correlation between the photons produced by the write and read pulses, the output of D1 is fed into the "Start" input of a time-interval analyzer, and the outputs of D2 and D3 are fed into two "Stop" inputs. A coincidence window imposed by the data acquisition software selects a time interval between the arrival of the idler and signal of $(0,80) \mathrm{ns}$ for $\Delta t=100 \mathrm{~ns}$ and $(25,145) \mathrm{ns}$ for $\Delta t=200 \mathrm{~ns}$.

We first measure the conditional probabilities of detecting a certain state of the idler (hence, of the quantum memory state) in the basis of $|H\rangle_{i}$ and $|V\rangle_{i}$, given the observed 
Table 1: Conditional probabilities $P(I \mid S)$ to detect the idler photon in state $I$ given detection of the signal photon in state $S$, at the point of maximum correlation for $\Delta t=100 \mathrm{~ns}$ delay between read and write pulses; all the errors are statistical.

\begin{tabular}{ccccc} 
Basis & $P\left(H_{i} \mid H_{s}\right)$ & $P\left(V_{i} \mid H_{s}\right)$ & $P\left(V_{i} \mid V_{s}\right)$ & $P\left(H_{i} \mid V_{s}\right)$ \\
\hline 0 & $0.92 \pm 0.02$ & $0.08 \pm 0.02$ & $0.88 \pm 0.03$ & $0.12 \pm 0.03$ \\
45 & $0.75 \pm 0.02$ & $0.25 \pm 0.02$ & $0.81 \pm 0.02$ & $0.19 \pm 0.02$
\end{tabular}

state of the signal photon. Varying the angle $\theta_{s}$ produces the correlation patterns shown in Fig.21A for $\Delta t=100 \mathrm{~ns}$. Conditional probabilities at the point of maximum correlation are shown in Fig.21B and the first line of Table 1. To verify faithful memory preparation and read-out, we repeat the correlation measurement in a different basis, of states $\left(|H\rangle_{i} \pm\right.$ $\left.|V\rangle_{i}\right) / \sqrt{2}$, by choosing the $\theta_{i}=45$ degrees, $\phi_{i}=0$ degrees, and $\phi_{s}=-\left(\eta_{s}+\eta_{i}\right)$ in the state transformers $R_{s}$ and $R_{i}$. We vary $\theta_{s}$, with the measured interference fringes displayed in Fig. 22A. Table 1 (second line) and Fig. 22B show the conditional probabilities at the point of maximum correlations. These probabilities are different from $1 / 2$ only when the phase coherence between the two states of the atomic qubit is preserved in the matter-to-light quantum state mapping.

From these measured correlations, we determine the fidelity of the reconstruction of our intended quantum memory state $\left|\Psi_{I}\right\rangle$ in the idler, $\left|\left\langle\Psi_{I} \mid \Psi_{i}\right\rangle\right|^{2}$. The fidelity is given by the value of the corresponding conditional probability at the point of maximum correlation, presented in Table 1 (we choose the lower of the two values as the lower bound). For states in the $\theta_{i}=0$ degree basis, we find $F_{0}=0.88 \pm 0.03$, clearly exceeding the classical boundary of $2 / 3$ [92]. For the $\theta_{i}=45$ degree basis, we found $F_{45}=0.75 \pm 0.02$, again significantly violating the classical limit. These fidelities give a lower bound for both the fidelities of the memory preparation and the read-out steps, which we do not measure separately.

Another way to quantify the performance of our quantum state transfer is to calculate the fidelity of entanglement between the signal and idler photons $F_{s i}$. The lower bound on $F_{s i}$ is given by the overlap of the measured density matrix with the maximally entangled state we seek to achieve $\left|\Psi_{M}\right\rangle$ given by Eq.27: $F_{s i}=\left\langle\Psi_{M}\left|\rho_{s i}\right| \Psi_{M}\right\rangle$ [93]. We calculated 
A

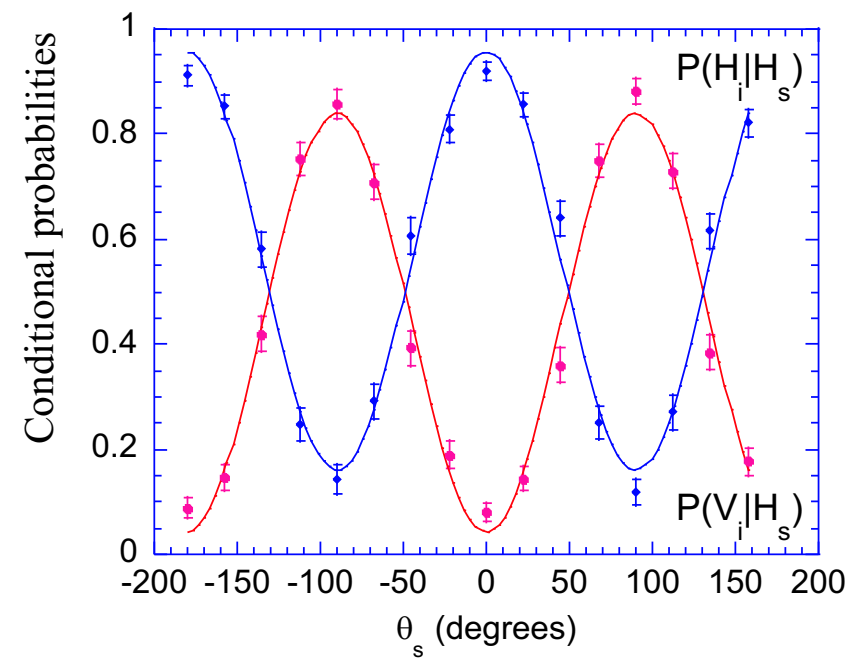

B

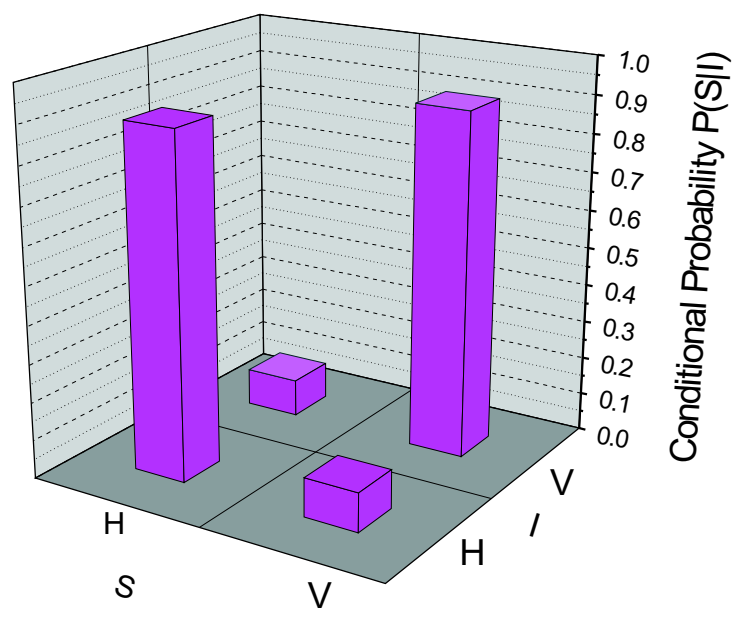

Figure 21: (A) Measured conditional probabilities $P\left(H_{i} \mid H_{s}\right)$ and $P\left(V_{i} \mid H_{s}\right)$ as the function of the polarization rotation $\theta_{s}$ of the signal photon. The full curves are fits with the visibility as the only adjustable parameter. (B) Measured conditional probabilities at the points of highest correlation. 
A

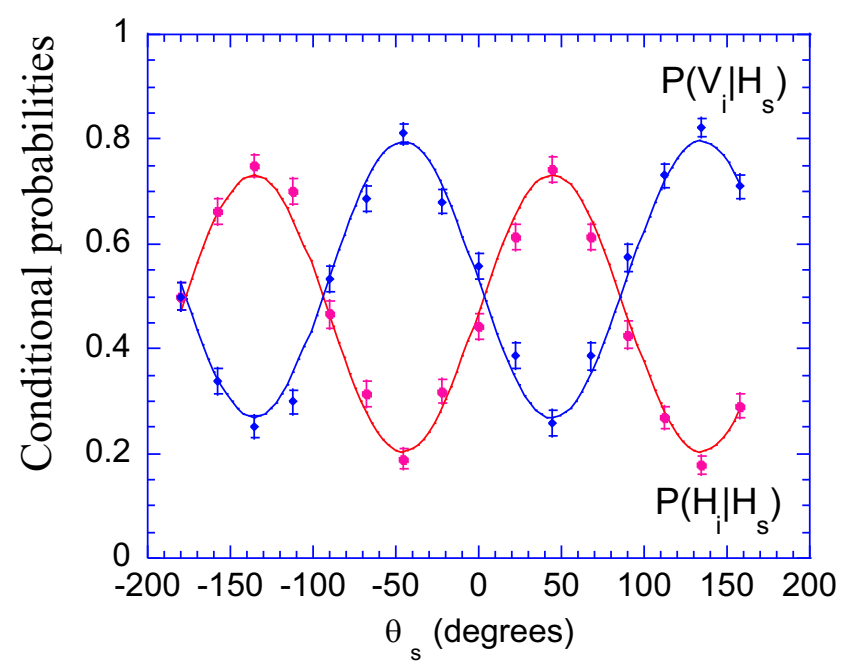

B

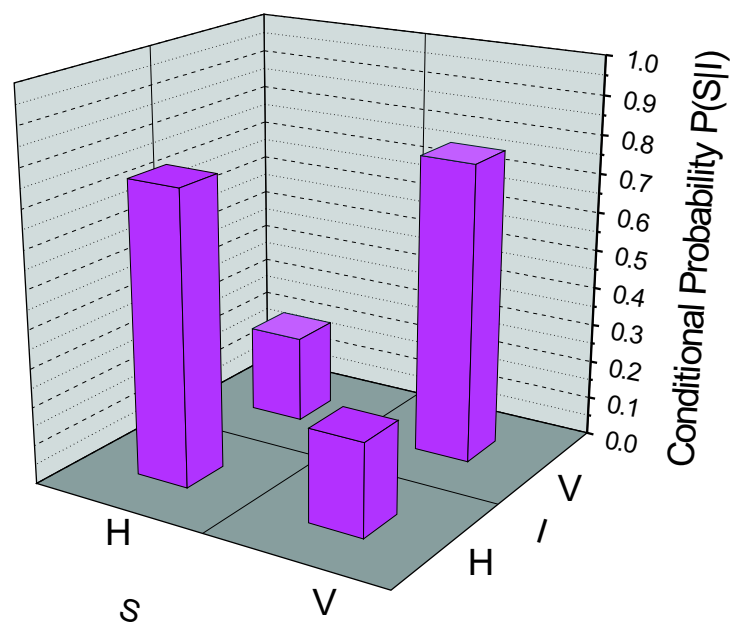

Figure 22: (A) Measured conditional probabilities after $\theta_{i}=\pi / 4$ polarization rotation of the idler photon as the function of $\theta_{s}$. (B) Measured conditional probabilities at the points of highest correlation. 


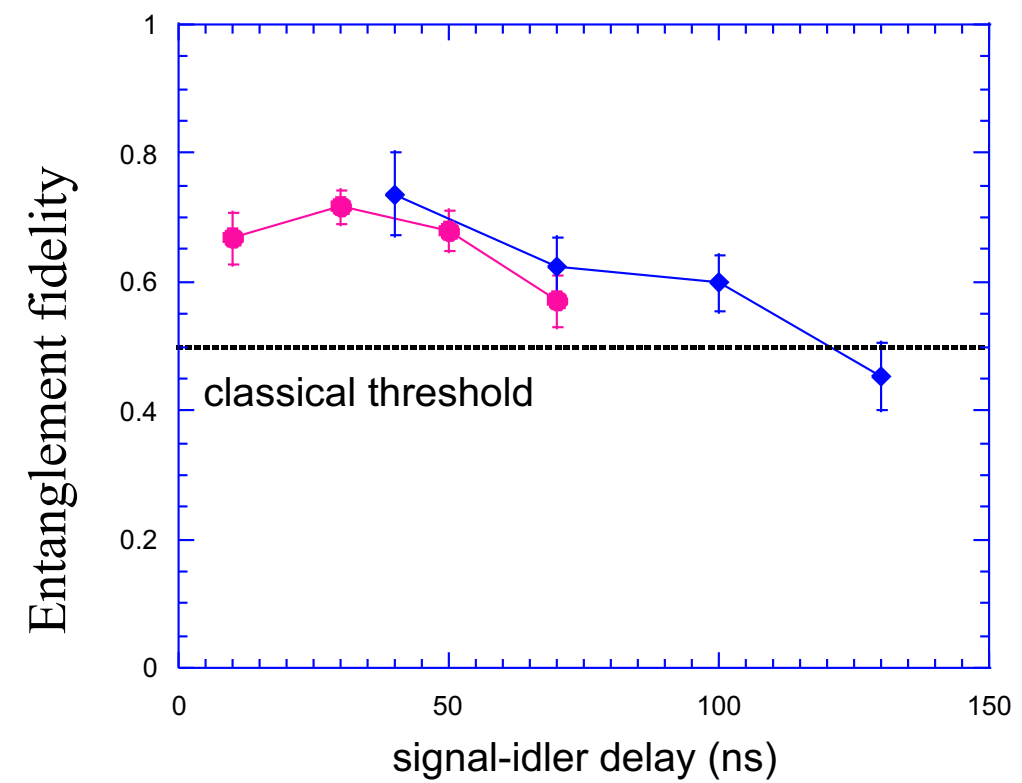

Figure 23: Time-dependent entanglement fidelity of the signal and the idler $F_{s i}$; circles for $\Delta t=100 \mathrm{~ns}$, diamonds for $\Delta t=200 \mathrm{~ns}$.

$F_{s i}=0.67 \pm 0.02$, substantially greater that the classical limit of $1 / 2[16,93]$.

At a longer delay of $200 \mathrm{~ns}$ the fidelities in the $\theta_{i}=0$ degrees and $\theta_{i}=45$ degrees bases are $F_{0}=0.79 \pm 0.04$ and $F_{45}=0.74 \pm 0.04$, while fidelity of entanglement is $F_{s i}=0.63 \pm 0.03$. For both values of $\Delta t$, we analyze the fidelity of entanglement as a function of the delay between the detections of the signal and the idler. We split the full coincidence window into four equal intervals, and calculated entanglement of formation for each one (Fig.23). From these results, we conclude that our quantum memory has a useful operational time of about 150 ns. The lifetime of coherence between the levels $|a\rangle$ and $|b\rangle$ determines the lifetime of the quantum memory and is limited by the magnetic field of the trapping quadrupole field of the MOT [43].

Non-zero coincidence counts in the minima of Fig. 21A are due to transmission losses and non-ideal spatial correlations between the signal and idler photons. The residual interferometric drifts in $\eta_{s}+\eta_{i}$ further reduce the visibility of Fig. 22A compared to Fig. $21 \mathrm{~A}$, resulting in a degradation of the fidelities. Losses also reduce the rate of entanglement generation. The rate of signal photon detections (and hence, atomic qubit preparation) is given by $R_{s}=\alpha n_{s} R \simeq 300 s^{-1}$, where $\alpha=0.05$ is the measured transmission efficiency for 
the write beam (which includes 0.60 detection efficiency), and $R=4.7 \times 10^{5} \mathrm{~s}^{-1}$ is the repetition rate of the experiment. Therefore, the inferred average photon number in the forward scattered mode per pulse is $n_{s} \simeq 1.4 \times 10^{-2}$. The coincident signal-idler detection rate is $R_{s i}=\zeta R_{s}=\zeta \alpha n_{s} R \simeq 0.4 s^{-1}$, where $\zeta \equiv \beta \xi \simeq 1.1 \times 10^{-3}$. The measured transmission and detection efficiency for the read beam is $\beta \simeq 0.04$, so we infer the efficiency of quantum state transfer from the atoms onto the photon $\xi \simeq 0.03$.

We have realized a quantum node by combining the entanglement of an atomic and photonic qubits with the atom-photon quantum state transfer. By implementing the second node at a different location, and performing a joint detection of the signal photons from the two nodes, the quantum repeater protocol [11], as well as distant teleportation of an atomic qubit may be realized. Based on this work, we estimate the rate for these protocols to be $R_{2} \simeq\left(\beta \xi \alpha n_{s}\right)^{2} R \simeq 3 \times 10^{-7} s^{-1}$. However, improvements in $\xi$ that are based on increasing the optical thickness of atomic samples [91], as well as elimination of transmission losses could provide several orders of magnitude increase in $R_{2}$. Our results also demonstrate the possibility of realizing quantum nodes consisting of multiple atomic qubits by using multiple beams of light. This approach shows promise for implementation of distributed quantum computation $[94,95]$. 


\section{CHAPTER VI}

\section{ENTANGLEMENT OF A PHOTON AND A COLLECTIVE ATOMIC EXCITATION}

This chapter is based on Ref. [47].

\subsection{Motivation}

In this Chapter we report probabilistic entanglement of a collective atomic excitation and a photon (signal), achieved using the off-axis, counter-propagating geometry of Braje et al. [53]. We propose and experimentally implement here a qubit consisting of two distinct mixed states of collective ground-state hyperfine coherence which contain one spin excitation. The entanglement of the signal photon and the collective spin excitation is inferred by performing quantum state transfer of the atomic qubit onto a photonic qubit (idler) [47], with one of the atomic states being converted into a right-hand polarized photon and the other into a left-hand polarized one. Polarization correlations of the signal and the idler photons are subsequently recorded and found to be in violation of the Bell inequality. The atom-photon entanglement is probabilistic, with the fundamental quantum state consisting mostly of vacuum. The entangled component of the state is postselected by coincidence counting. This type of entanglement is similar to two-photon entanglement in spontaneous parametric down-conversion (see [96, 97, 30] and references therein), and to the ion-photon entanglement of Blinov et al. $[16,17]$.

\subsection{Theory}

As illustrated in Fig.24(a), the right circularly polarized write pulse generates a cone of

forward Raman scattering. We collect a Gaussian mode centered around the momentum $\vec{k}_{s}$ that forms a $2^{\circ}$ angle with the write beam. Fig.24(b) indicates schematically the structure of the three atomic levels involved, $|a\rangle,|b\rangle$ and $|c\rangle$. The experimental sequence starts with 


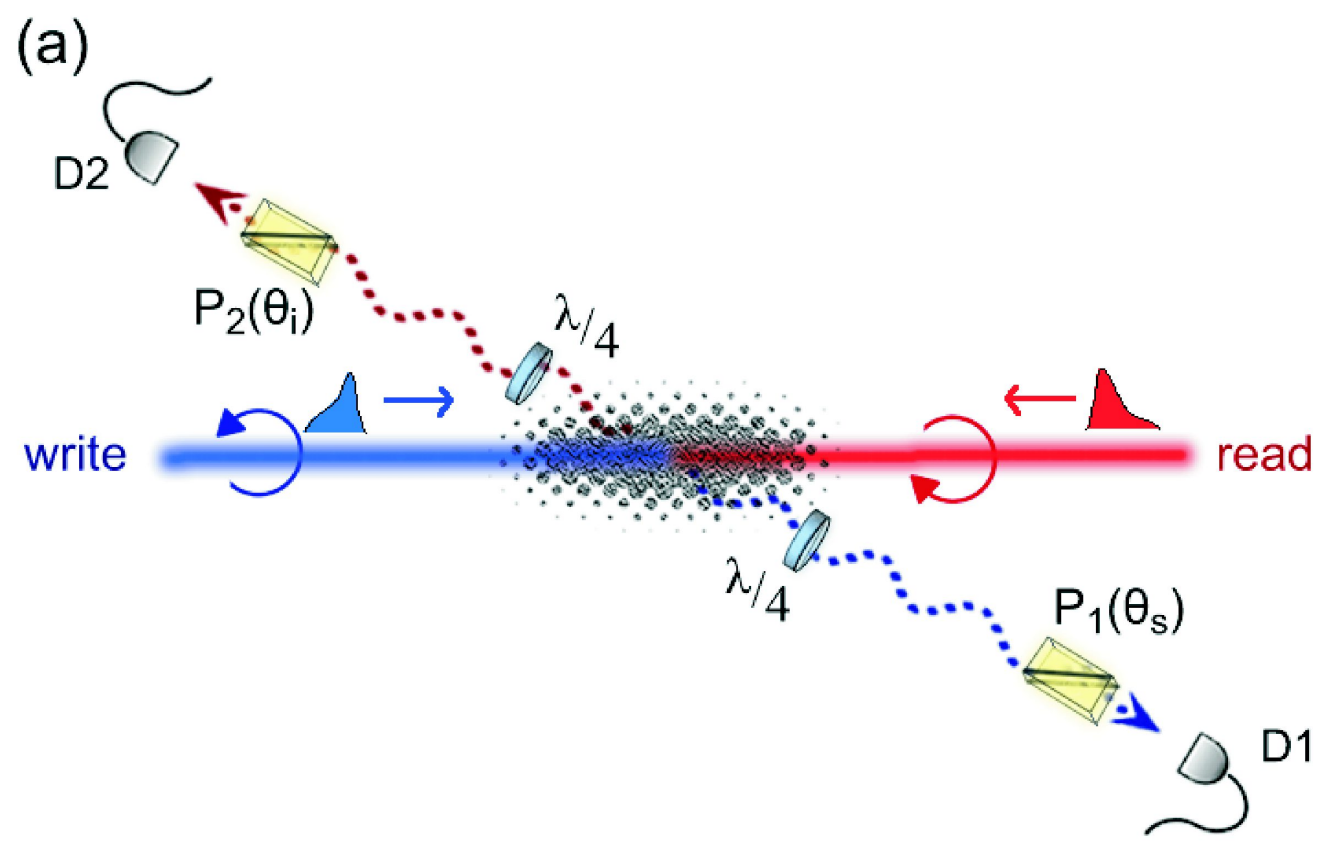

(b)

write stage read stage

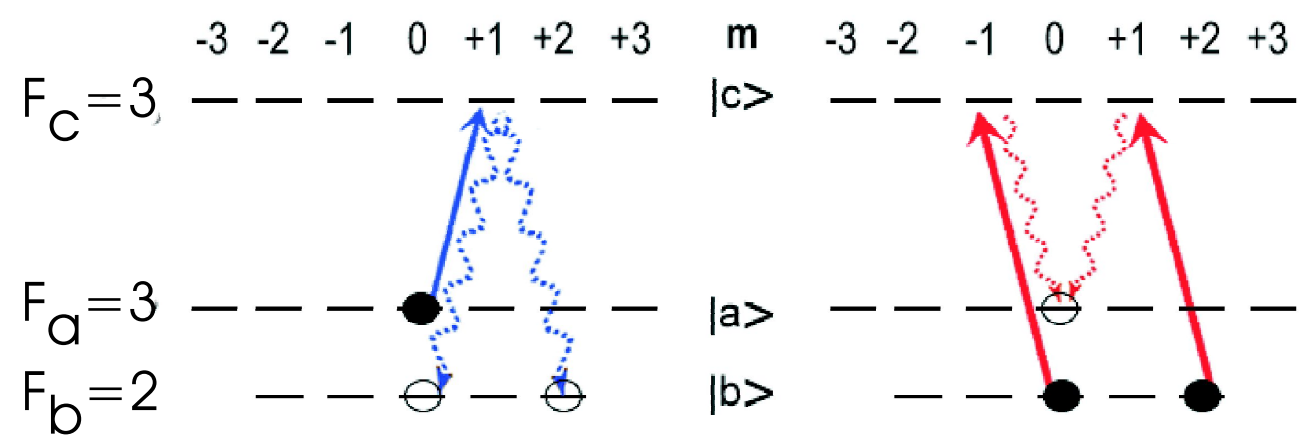

Figure 24: (a) Schematic of experimental setup. $P_{1}$ and $P_{2}$, polarizers; D1 and D2, detectors; $\lambda / 4$, quarter-waveplate. (b) The structure of atomic transitions leading to generation of atom-photon entanglement and of the subsequent read-out of atomic qubit. 
all of the atoms prepared in the unpolarized level $|a\rangle$. A write pulse tuned to the $|a\rangle \rightarrow|c\rangle$ transition is directed into a sample of cold ${ }^{85} \mathrm{Rb}$ atoms. The classical write pulse is so weak that less than one photon is scattered in this manner on the $|c\rangle \rightarrow|b\rangle$ transition into the collected mode for each pulse.

Perturbation theory shows that the ensemble-photon density operator may be written as $|v a c\rangle\langle v a c| \otimes \rho_{a}+\epsilon \rho$ where $\rho$ has unit trace, and $\epsilon<<1$. Here $|v a c\rangle$ is the photon vacuum state and $\rho_{a}$ the atomic ensemble vacuum state density operator, corresponding to $N$ atoms each populating the Zeeman states $|a, m\rangle$ of level $|a\rangle$ with equal probability $1 /\left(2 F_{a}+1\right)$. It is important to realize that the vacuum component in state $|v a c\rangle\langle v a c| \otimes \rho_{a}+\epsilon \rho$ has no influence on the fidelity of DLCZ's quantum communication protocols due to built-in purification, even though $\epsilon \ll 1[11]$. Writing $|r\rangle$ and $|l\rangle$ as the normalized states of right and left circular polarization of the signal photon propagating towards the detector in direction $\vec{k}_{s}$, we have that, in the ideal case

$$
\begin{aligned}
\rho= & \cos ^{2} \eta|r\rangle\left\langle r\left|\hat{s}_{-1}^{\dagger} \rho_{a} \hat{s}_{-1}+\sin ^{2} \eta\right| l\right\rangle\langle l| \hat{s}_{1}^{\dagger} \rho_{a} \hat{s}_{1} \\
+ & \cos \eta \sin \eta\left(|r\rangle\left\langle l\left|\hat{s}_{-1}^{\dagger} \rho_{a} \hat{s}_{1}+\right| l\right\rangle\langle r| \hat{s}_{1}^{\dagger} \rho_{a} \hat{s}_{-1}\right)
\end{aligned}
$$

where

$$
\cos ^{2} \eta=\sum_{m} X_{m}^{2}(-1) /\left[\sum_{m} \sum_{\alpha= \pm 1} X_{m}^{2}(\alpha)\right]
$$

with $m$ summed over $\left\{-F_{a}, \ldots F_{a}\right\}$, and $X_{m}(\alpha)=C_{m, 1, m+1}^{F_{a}, 1, F_{c}} C_{m+1, \alpha, m+\alpha+1}^{F_{c}, 1, F_{b}}$ is the product of the relevant Clebsch-Gordan coefficients for the transition. The collective atomic spin excitation operators are given by

$$
\hat{s}_{\alpha}^{\dagger}=\sum_{m}\left(\frac{X_{m}(\alpha)}{\sqrt{\sum_{m} X_{m}^{2}(\alpha)}}\right) \hat{s}_{\alpha}^{\dagger}(m)
$$

and

$$
\hat{s}_{\alpha}^{\dagger}(m)=\sqrt{\frac{2 F_{a}+1}{N}} \sum_{\mu=1}^{N} e^{-i \Delta \vec{k}_{s} \cdot \vec{r}_{\mu}}|b, m+1+\alpha\rangle_{\mu}\langle a, m|,
$$

where $\Delta \vec{k}_{s}=\vec{k}_{s}-\vec{k}_{w}$, is the difference in the signal and write beam wave vectors and $\vec{r}_{\mu}$ is the position of atom $\mu$. For weak states of excitation the collective spin operators satisfy bosonic commutation relations correct to $O(1 / N):\left[\hat{s}_{\alpha}(m), \hat{s}_{\alpha^{\prime}}^{\dagger}\left(m^{\prime}\right)\right]=\delta_{\alpha, \alpha^{\prime}} \delta_{m, m^{\prime}}$ and 
$\left[\hat{s}_{\alpha}, \hat{s}_{\alpha^{\prime}}^{\dagger}\right]=\delta_{\alpha, \alpha^{\prime}}$. Evaluating the coefficient $\cos ^{2} \eta$ for the experimental conditions $F_{a}=F_{c}=$ $3, F_{b}=2$, we find $\eta=0.81 \times \pi / 4$.

Detection of a photon by D1 produced by the $|c\rangle \rightarrow|b\rangle$ transition results in the sample of atoms containing, in the ideal case, exactly one excitation in the related collective atomic mode. After a variable delay time $\Delta t$ (bounded by the lifetime of the ground-state atomic coherences) we convert the atomic excitation into a single photon by illuminating the atomic ensemble with a pulse of light near-resonant with the $|b\rangle \rightarrow|c\rangle$ transition and counterpropagating with respect to the write beam (Fig.24). For an optically thick atomic sample, the idler photon will be emitted with high probability into the mode determined by the phase-matching condition $\vec{k}_{i}=\vec{k}_{w}+\vec{k}_{r}-\vec{k}_{s}$, with the atomic qubit state mapped onto a photonic one. Under the condition of collective enhancement the atomic excitations generated by $\hat{s}_{ \pm 1}^{\dagger}$ map to orthogonal idler photon states up to a phase. Assuming equal mapping efficiency, the number of correlated signal-idler counts registered by the detectors can be predicted on the basis of Eq. (28). We find, by carefully analyzing the measurement procedure,

$$
\begin{array}{r}
C\left(\theta_{s}, \theta_{i}\right) \propto\left[(\cos \eta+\sin \eta) \cos \left(\theta_{s}-\theta_{i}\right)+\right. \\
\left.(\cos \eta-\sin \eta) \cos \left(\theta_{s}+\theta_{i}\right)\right]^{2},
\end{array}
$$

where $\theta_{s}$ and $\theta_{i}$ are the orientations of polarizers $P_{1}$ and $P_{2}$. Following Clauser-HorneShimony-Holt (CHSH) $[98,75]$, we calculate the correlation function $E\left(\theta_{s}, \theta_{i}\right)$, given by

$$
\frac{C\left(\theta_{s}, \theta_{i}\right)+C\left(\theta_{s}^{\perp}, \theta_{i}^{\perp}\right)-C\left(\theta_{s}^{\perp}, \theta_{i}\right)-C\left(\theta_{s}, \theta_{i}^{\perp}\right)}{C\left(\theta_{s}, \theta_{i}\right)+C\left(\theta_{s}^{\perp}, \theta_{i}^{\perp}\right)+C\left(\theta_{s}^{\perp}, \theta_{i}\right)+C\left(\theta_{s}, \theta_{i}^{\perp}\right)},
$$

where $\theta^{\perp}=\theta+\pi / 2$. The CHSH version of the Bell inequality is then $|S| \leq 2$ where

$$
S=E\left(\theta_{s}, \theta_{i}\right)+E\left(\theta_{s}^{\prime}, \theta_{i}\right)+E\left(\theta_{s}, \theta_{i}^{\prime}\right)-E\left(\theta_{s}^{\prime}, \theta_{i}^{\prime}\right)
$$

The maximum violation of the Bell inequality is achieved for a maximally entangled state with the canonical set of angles $\theta_{s}=-22.5^{\circ}, \theta_{i}=0^{\circ}, \theta_{s}^{\prime}=22.5^{\circ}$ and $\theta_{i}^{\prime}=-45^{\circ}: S=2 \sqrt{2}=$ 2.83. Based on the value $\eta=0.81 \times \pi / 4$ we find, ideally, $S=2.77$ which significantly violates the Bell inequality. 
Table 2: Measured correlation function $E\left(\theta_{s}, \theta_{i}\right)$ and $S$ for $\Delta t=200$ ns delay between write and read pulses; all the errors are based on the statistics of the photon counting events.

\begin{tabular}{ccc}
$\theta_{s}$ & $\theta_{i}$ & $E\left(\theta_{s}, \theta_{i}\right)$ \\
\hline-22.5 & 0 & $0.641 \pm 0.024$ \\
-22.5 & -45 & $0.471 \pm 0.029$ \\
22.5 & 0 & $0.587 \pm 0.027$ \\
22.5 & -45 & $-0.595 \pm 0.027$ \\
& & $S=2.29 \pm 0.05$
\end{tabular}

\subsection{Experiment}

A magneto-optical trap (MOT) of ${ }^{85} \mathrm{Rb}$ is used to provide an optically thick atomic cloud for our experiment (Fig.24). The ground levels $\{|a\rangle ;|b\rangle\}$ correspond to the $5 S_{1 / 2}, F_{a, b}=\{3,2\}$ levels, while the excited level $|c\rangle$ represents the $\left\{5 P_{1 / 2}, F_{c}=3\right\}$ level of the $D_{1}$ line at 795 nm. The experimental sequence starts with all of the atoms prepared in level $|a\rangle$. The "dark" period lasts $640 \mathrm{~ns}$, with the whole cycle taking $1.5 \mu \mathrm{s}$. All the light responsible for trapping and cooling is shut off during the dark period, with the trapping light shut off about 200 ns before the repumping light to empty the $F=2$ hyperfine level. The quadrupole magnetic field of the MOT is switched off for the duration of the measurement sequence. The ambient magnetic field is compensated by three pairs of Helmholtz coils.

A 130 ns long write pulse tuned to the $|a\rangle \rightarrow|c\rangle$ transition is focused into the MOT with a Gaussian waist of about $400 \mu \mathrm{m}$. The light induces spontaneous Raman scattering via the $|c\rangle \rightarrow|b\rangle$ transition. The scattered light goes through the quarter-wave plate to map circular polarizations into linear ones, then passes through polarizer $P_{1}$ (set at angle $\theta_{s}$ ) and impinges onto a single photon detector D1.

After a user-programmable delay $\Delta t$, a 120 ns long read pulse, with circular polarization opposite to that of the write pulse, tuned to the $|b\rangle \rightarrow|c\rangle$ transition illuminates the atomic ensemble. This accomplishes a transfer of the memory state onto the single photon (idler) emitted by the $|c\rangle \rightarrow|a\rangle$ transition. After passing through the quarter-wave plate and polarizer $P_{2}$ set at angle $\theta_{i}$, the idler photon is directed onto a single-photon detector D2.

Both write/read and signal/idler pairs of fields are counter-propagating. The waist of 


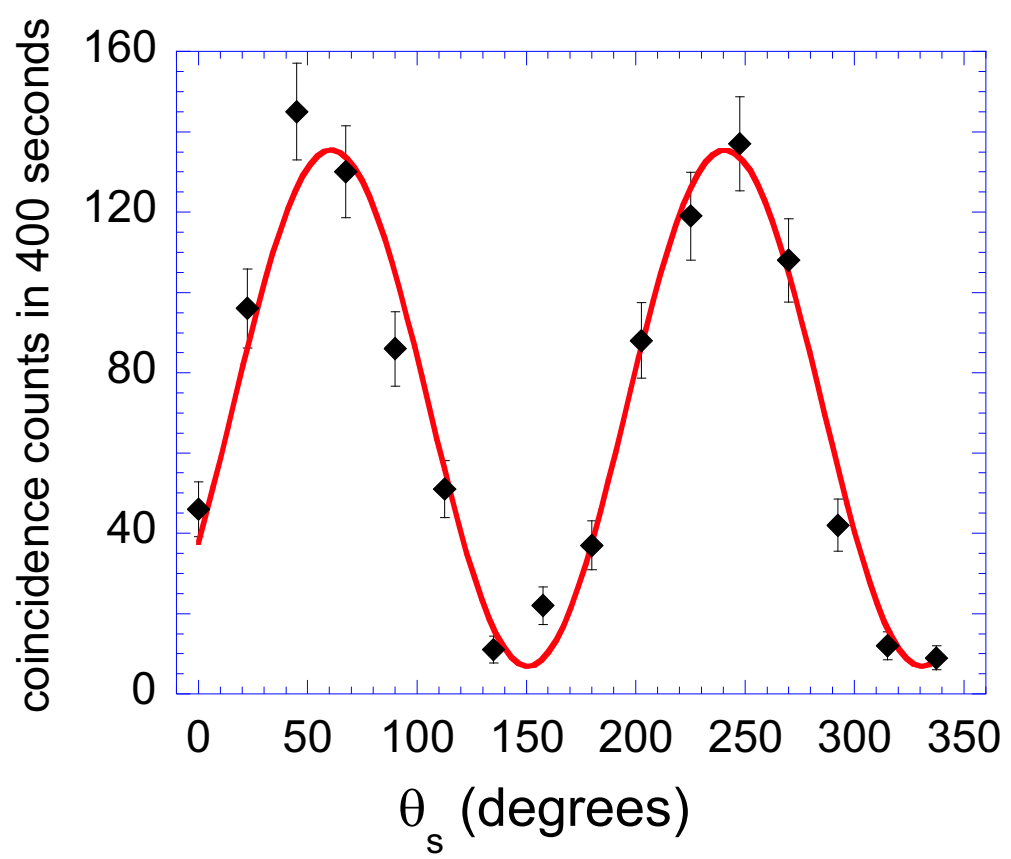

Figure 25: Measured coincidence fringe for $\theta_{i}=67.5^{\circ}$. The curve is a fit based on Eq.(5), augmented by a background contribution, with $\eta=0.81 \times \pi / 4$, with visibility and amplitude being adjustable parameters. The visibility of the fit is $90 \%$. Uncertainties are based on the statistics of the photon counting events.

the signal-idler mode in the MOT is about $150 \mu \mathrm{m}$. The four-wave mixing signal is used to align the single mode fibers collecting signal and idler photons, and to optimize the overlap between the pump and probe modes [53]. The value of delay $\Delta t$ between the application of the write and read pulses is $200 \mathrm{~ns}$. The electronic pulses from the detectors are gated with $140 \mathrm{~ns}$ and $130 \mathrm{~ns}$ windows centered on the time determined by the write and read light pulses, respectively. Afterwards, the electronic pulses are fed into a time-interval analyzer (with 2 ns time resolution). In order to measure the correlation between the photons produced by the write and read pulses, the output of D1 is fed into the "Start" input of a time-interval analyzer, and the output of D2 is fed into the "Stop" input.

A typical interference fringe in the signal-idler coincidence detection is displayed in Fig.25. In order to infer probabilistic atom-photon entanglement, we calculate the degree of Bell inequality violation $|S| \leq 2[98,75]$. Table 2 presents measured values for the correlation function $E\left(\theta_{s}, \theta_{i}\right)$ using the canonical set of angles $\theta_{s}, \theta_{i}$. We find $S=2.29 \pm 0.05 \not \leq 2$ a clear violation of the Bell inequality. The value of $S$ is smaller than the ideal value of 2.77 due to experimental imperfections, particularly non-zero counts in the minima of 


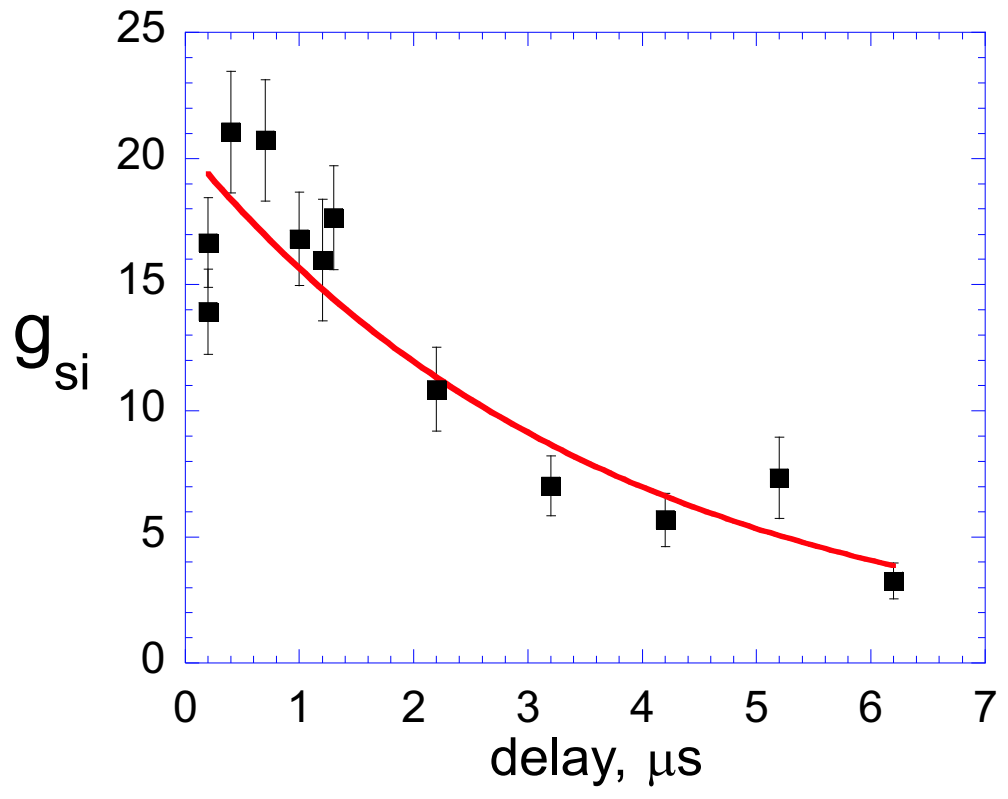

Figure 26: Normalized signal-idler intensity correlation function $g_{s i}$ as a function of storage time. Uncertainties are based on the statistics of the photon counting events. The full curve is the best exponential fit with time constant $\tau=3.7 \mu \mathrm{s}$.

interference curves that arise as the result of the finite value of the normalized signal-idler intensity correlation function $g_{s i}[30,43,99]$ shown in Fig.26. To our knowledge, this is the first observed violation of the Bell inequality involving a collective excitation.

The effective detection efficiencies as determined by the ratios of the coincidence signalidler count rate $R_{s i}$ to singles count rates $R_{s}$ and $R_{i}$ are $\alpha_{s, i}=R_{s i} / R_{i, s} \simeq 0.02$. In all cold atomic ensemble experiments within the DLCZ program reported to date, the quadrupole magnetic field of the MOT has been the main source of the atomic memory decoherence (limiting storage times on the order of $100 \mathrm{~ns}[43,99,100,49])$. In this work, we have switched off the quadrupole field for the duration of our protocol, and the coherence time has increased to several $\mu \mathrm{s}$, as is evident from the measured normalized intensity correlation function $g_{s i}$ displayed in Fig.26 (the length of the dark period was increased up to $7 \mu$ s for this measurement at the expense of lower count rate). 


\section{CHAPTER VII}

\section{ENTANGLEMENT OF REMOTE ATOMIC QUBITS}

Part of this chapter is based on Ref. [101].

\subsection{Introduction}

Realization of massive qubits, and their entanglement, is central to practical quantum information systems $[2,11,3]$. Remote entanglement of photons can now be achieved in a robust manner using the well-developed technology of spontaneous parametric downconversion $[6,7,102]$, with propagation to remote locations by means of optical fibers. Photons, however, are difficult to store for any appreciable period of time, whereas qubits based on ground-state atoms have long lifetimes. Local entanglement of massive qubits has been observed between adjacent trapped ions [103] and between pairs of Rydberg atoms in a collimated beam [15]. In order to entangle qubits at remote locations the use of photons as an intermediary seems essential [104, 105, 106, 107]. Photons also offer some flexibility as information carriers as they can propagate in optical fiber with low losses. The creation, transport, storage, and retrieval of single photons between remote atomic ensembles located in two different laboratories was reported in Chapter 3, (see also [48]). The first step in creating remote entanglement between massive qubits is to entangle one such qubit with the mediating light field, which is then directed towards the second qubit via an optical fiber. There have recently been important advances towards this goal by demonstrating entanglement of a photon with a trapped ion [16], with a collective atomic qubit [46, 47], and with a single trapped atom [19].

A promising route towards the creation and application of long-lived qubit entanglement in scalable quantum networks was proposed by Duan, Lukin, Cirac, and Zoller [11, 107]. These atomic qubits rely on collective atomic states containing exactly one spin excitation. For useful quantum information processing two orthogonal spin wave excitation states 
$\hat{s}_{+}^{\dagger}|0\rangle_{a}, \hat{s}_{-}^{\dagger}|0\rangle_{a}$ are needed for the logical states of a qubit [11], where $|0\rangle_{a}$ represents the collective atomic ground state. In the experiment of Ref.[48] each of the two remote ensembles only contained one logical state, since the atomic ground state component does not serve this purpose. Entanglement of continuous atomic variables in two separate atomic ensembles has been reported [39], as appropriate for continuous-variable quantum information processing, but not for qubit entanglement.

In two experiments edscribed in Chapters 5 and 6 , collective atomic qubits were generated using cold atomic ensembles [46, 47]. In the first of these the logical states were

single spin wave excitations (ideally, $\hat{s}_{+}^{\dagger}|0\rangle_{a}, \hat{s}_{-}^{\dagger}|0\rangle_{a}$ ), in either one of two distinct atomic ensembles inside a high vacuum chamber. In the second experiment, two orthogonal spin waves of a single cold ensemble represented the logical qubit states [47]. The experiments $[46,47]$ realized a single atomic qubit system, but did not address the issue of entanglement of atomic qubits.

While remote entanglement of atomic qubits has not been previously demonstrated, Chapters 5 and 6 describe the realisation of two basic primitives of a quantum network: (a) entanglement of photonic and atomic qubits, and (b) quantum state transfer from an atomic to a photonic qubit. The crucial additional ingredient is the reverse operation, the conversion of a photonic qubit into an atomic qubit. This enables the transfer of atomphoton entanglement into remote atomic qubit entanglement.

\subsection{Experiment}

Here we report remote atomic qubit entanglement using cold atomic clouds of ${ }^{85} \mathrm{Rb}$ confined at Sites $A$ and $B$, as shown in Fig. 27. These sites are situated in separate laboratories and linked by an optical fiber. A notable distinction between the two nodes is that the qubit generated at Site $A$ is written on an unpolarized atomic ensemble, as in Ref. [47], whereas at Site $B$ the atomic ensemble is prepared, ideally, in the $(m=0)$ Zeeman state of the $F=2$ ground level by optical pumping. All the light fields responsible for trapping and cooling of the atoms, as well as the quadrupole magnetic fields at both sites, are shut off during the period of the protocol. The ambient magnetic field at each site is compensated 
by three pairs of Helmholtz coils, and a bias field of $0.2 \mathrm{G}$ is added at Site $B$ for the purpose of optical pumping.

Our protocol starts with the generation of an entangled state of a signal photon and a collective atomic qubit at Site $A$, achieved through Raman scattering of a classical laser write pulse. The state can be represented schematically as

$$
\begin{aligned}
|\Psi\rangle & =|0\rangle_{a}|0\rangle_{f}+\chi\left(\cos \eta|+\rangle_{a}|+\rangle_{f}+\sin \eta|-\rangle_{a}|-\rangle_{f}\right) \\
& \equiv|0\rangle_{a}|0\rangle_{f}+\chi|\psi\rangle
\end{aligned}
$$

where $|+\rangle_{f} \equiv \hat{a}_{+}^{\dagger}|0\rangle_{f}$ and $|-\rangle_{f} \equiv \hat{a}_{-}^{\dagger}|0\rangle_{f}$ are the normalized states of positive and negative helicity of the signal photon, $|0\rangle_{f}$ is the field vacuum state, $| \pm\rangle_{a} \equiv \hat{s}_{ \pm}^{\dagger}|0\rangle_{a}$ describes the two logical qubit states, corresponding to non-symmetric collective atomic modes [63], and $\chi<<$ 1. The asymmetry angle $\eta=0.81 \pi / 4$ [47]. Eq.(35) represents probabilistic entanglement generation, where ideally for each signal photon emission event, an entangled atomic qubit is created in the atomic ensemble $[16,11]$. Since we deal with an unpolarized atomic ensemble, the state of the system is more rigourously described by a density operator as discussed in Ref.[47].

The orthogonal polarization modes of the signal field produced at Site $A$ are directed along the optical fiber to Site $B$. As the signal field propagates from Site $A$ to Site $B$, it passes through two quarter wave plates, causing the transformation of the signal field operators $\hat{a}_{ \pm} \rightarrow \pm \hat{a}_{\mp}$. The signal field propagation in the atomic medium at Site $B$ is controlled by an additional laser field (control) through the process of electromagnetically-induced transparency (EIT) $[40,64,41,42,26,27]$.

We implement the storage phase at Site $B$, by adiabatically reducing the control field amplitude to zero, while the signal pulse lies within the cloud. The orthogonal atomic spin wave excitations thereby created in the spin-polarized gas constitute the logical states of the atomic qubit. In order to convert the signal field qubit into a collective atomic qubit, it is necessary that the optically thick atomic sample supports EIT for both field helicities [42]. To this end, we optically pump the atomic cloud at Site $B$ using a linearly polarized field resonant to the $F=2 \leftrightarrow F^{\prime}=2$ transition of the $D_{1}$-line, and an additional repumping 


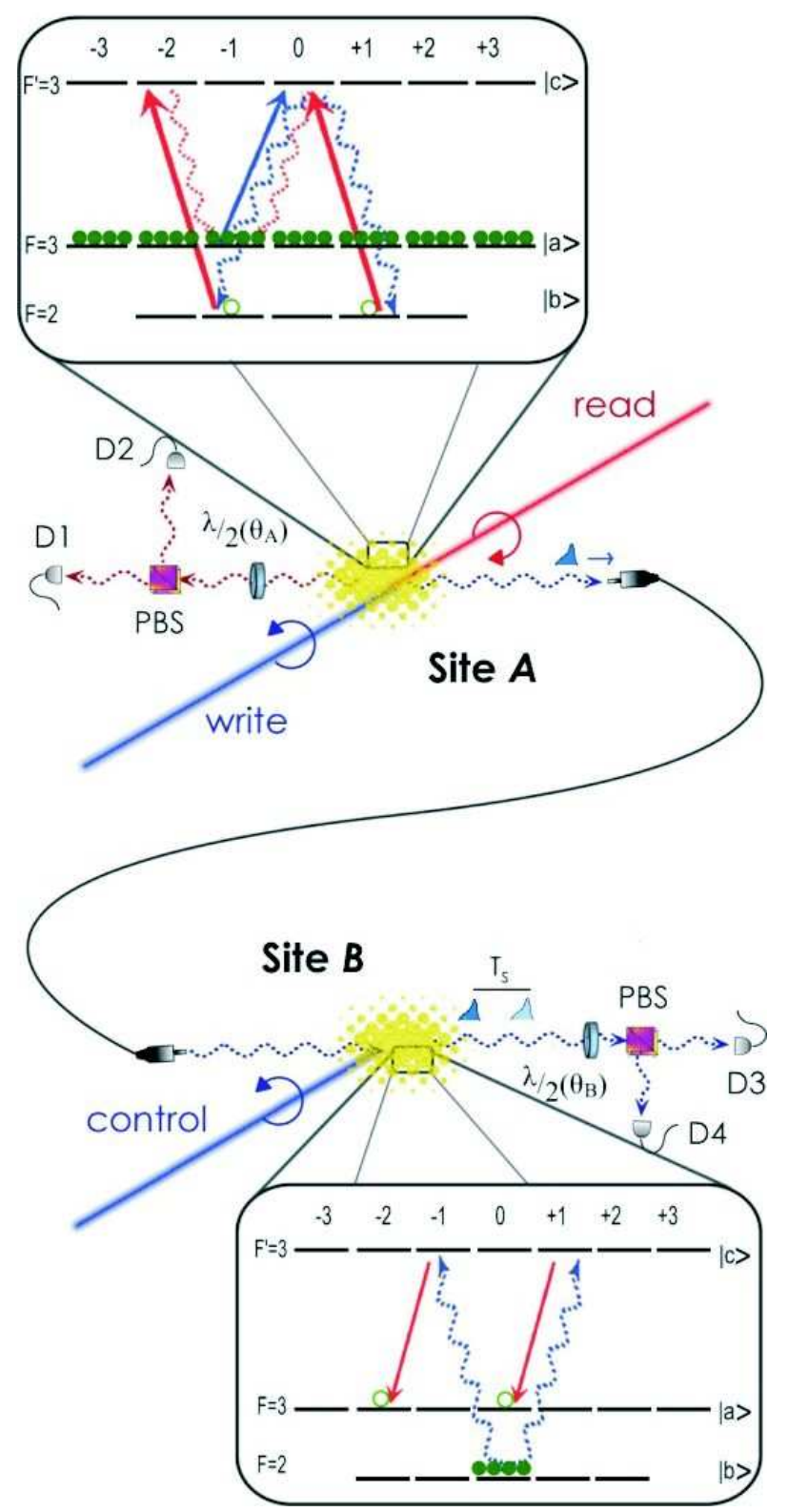

Figure 27: A schematic diagram of our experimental setup. Two cold atomic ensembles of ${ }^{85} \mathrm{Rb}$, an unpolarized sample at Site $A$, and a spin-polarized sample at Site $B$, separated by $5.5 \mathrm{~m}$, are connected by a single-mode fiber. The insets show the structure and the initial populations of the atomic levels for the two ensembles. An entangled state of a collective atomic qubit and a signal field is generated at Site $A$ by Raman scattering of the write laser field. The orthogonal helicity states of the generated signal field are transmitted via optical fiber from Site $A$ to Site $B$, where they are converted to orthogonal collective atomic excitations, stored for a duration $T_{s}$, and subsequently converted into an idler field by adiabatic variation of the control field amplitude. The atomic qubit at Site $A$ is similarly converted into an idler field by a read laser pulse, counterpropagating with respect to the write pulse. For polarization analysis, each idler field propagates through a quarter-wave plate (not shown), a half-wave plate $(\lambda / 2)$ and a polarizing beamsplitter (PBS). Polarization correlations of the idler fields are recorded by photoelectric detection using the single photon detectors D1-D4. 
field resonant to the $F=3 \leftrightarrow F^{\prime}=3$ transition of the $D_{2}$-line. We measured the optical thickness $d \simeq 8$ for both circular components of the signal field.

By switching off the control field over a period of about $20 \mathrm{~ns}$, the photonic qubit is converted into an atomic qubit. At this stage remote atomic qubits should have been created at Sites $A$ and $B$. Atoms at Site $B$ should, ideally, be prepared in a single Zeeman $m=0$ state of the $F=2$ hyperfine ground level (lower inset in Fig. 27). In practice the pumping is not perfect, possibly due to radiation trapping in the optically thick atomic medium [108]. We measure lower storage and retrieval efficiency for the negative helicity signal component compared with that of the positive helicity component (3\% vs $8 \%$ ). Numerical simulations indicate that the discrepancy between the efficiencies is consistent with a residual population in the $|F=2, m=-2\rangle$ atomic state at the $10 \%$ level [59]. This results in undesirable absorption of the signal field with negative helicity.

The signal photon of helicity $\alpha= \pm 1$ is stored in the ensemble at Site $B$ with efficiency $\epsilon_{\alpha}$. After a storage time $T_{s}$, the non-vacuum component of the state of the two ensembles is given by the following density operator: $\hat{\rho}=(1-\epsilon) \hat{\rho}_{A}+\epsilon \hat{\rho}_{A B}$, where the component $\hat{\rho}_{A}$ describes the state of single excitation at Site $A$, and is expressed by

$$
\hat{\rho}_{A}=\frac{1-\epsilon_{-}}{1-\epsilon} \cos ^{2} \eta \hat{s}_{A+}^{\dagger} \hat{\rho}_{v a c} \hat{s}_{A+}+\frac{1-\epsilon_{+}}{1-\epsilon} \sin ^{2} \eta \hat{s}_{A-}^{\dagger} \hat{\rho}_{v a c} \hat{s}_{A-}
$$

where $\hat{\rho}_{\text {vac }}$ is the product of the ground state atomic density operators for the ensembles at Sites $A$ and $B$. The density operator $\hat{\rho}_{A B}=\hat{\Psi}_{A B}^{\dagger}\left(T_{s}\right) \hat{\rho}_{v a c} \hat{\Psi}_{A B}\left(T_{s}\right)$ in the two-qubit sub-space represents an entangled atomic state where

$$
\hat{\Psi}_{A B}^{\dagger}\left(T_{s}\right)=e^{i \phi\left(T_{s}\right)} \cos \eta^{\prime} \hat{s}_{A+}^{\dagger} \hat{s}_{B-}^{\dagger}-\sin \eta^{\prime} \hat{s}_{A-}^{\dagger} \hat{s}_{B+}^{\dagger}
$$

with $\cos \eta^{\prime}=\sqrt{\epsilon_{-} / \epsilon} \cos \eta$, and $\epsilon=\epsilon_{-} \cos ^{2} \eta+\epsilon_{+} \sin ^{2} \eta$ is the average efficiency of photon storage at Site $B$. The phase $\phi(t)=-2\left(g \mu_{B} / \hbar\right) B_{0} t$ is induced by the applied magnetic field $B_{0}=0.2 \mathrm{G}$ oriented along the propagation axis at Site $B$, where $g$ is the Landé g-factor for hyperfine level with $F=3$.

Ideally, entanglement should have been created between the collective atomic qubits at Sites $A$ and $B$. After a storage time $T_{s}$, the remote collective atomic excitations are converted 
by quantum state transfer into idler fields emanating from Sites $A$ and $B$, using a read laser pulse at Site $A$ and by reactivating the control field at Site $B[46,47]$. The resulting idler-idler photoelectric correlations may be calculated using the effective two-photon state

$$
\left|\Psi_{2}\right\rangle=\cos \eta_{f}|H V\rangle+e^{i \phi_{f}} \sin \eta_{f}|V H\rangle
$$

where $|H V\rangle=\hat{a}_{A, H}^{\dagger} \hat{a}_{B, V}^{\dagger}|0\rangle_{f}$ and $|V H\rangle=\hat{a}_{A, V}^{\dagger} \hat{a}_{B, H}^{\dagger}|0\rangle_{f}$, and the subscripts $A$ and $B$ indicate the idler mode at the respective site. We omit higher-order terms in photon number $[48]$.

The phase $\phi_{f}$, which includes the contributions due to the Larmor precession $\phi\left(T_{s}\right)$, the light phase shifts in the atomic media, and various optical elements, is introduced as an adjustable parameter. The mixing angle $\eta_{f}$ is determined by the relative efficiencies with which the two qubit states are transferred from the atomic ensembles to the idler fields. If we assume equal transfer efficiencies at Site $A$, we find $\cos \eta_{f}=\sqrt{\epsilon_{B-} / \epsilon_{B}} \cos \eta$, where $\epsilon_{B}=\epsilon_{B-} \cos ^{2} \eta+\epsilon_{B+} \sin ^{2} \eta$ and $\epsilon_{B \pm}$ is the combined storage and retrieval efficiency for a photon of helicity \pm at Site $B$. Measurements of these efficiencies give $\epsilon_{B+}=0.08$, and $\epsilon_{B-}=0.03$. With $\eta=0.81 \pi / 4$ fixed by the atom-photon entanglement process at Site $A$ [47] we get $\eta_{f}=1.12 \pi / 4$. Our experimental data, including those displayed in Fig. 29, are consistent with this value of $\eta_{f}$ and $\phi_{f} \ll 1$.

The above arguments are clearly conditional on the generation of the signal qubit. According to Eq.(35), the corresponding probability scales as $\chi^{2}$, and this determines the efficiency of the probabilistic entanglement generation. However, as Duan et al. point out [11], quantum network protocols eliminate the vacuum component of Eq.(35) and only the entanglement characteristics of $|\psi\rangle$ are relevant [109, 110]. In our experiment, atomic qubits were stored for a time 500 ns at Site $A$ and 200 ns at Site $B$. It should be possible to extend the qubit storage times to longer than $10 \mu \mathrm{s}$, as the single-quanta storage results suggest [48].

The measurement of the atomic qubits is performed by quantum state transfer onto the idler fields at both sites, using the read laser pulse at Site $A$ and the control laser pulse at Site $B$. The polarization state of either idler field is measured using a polarizing 


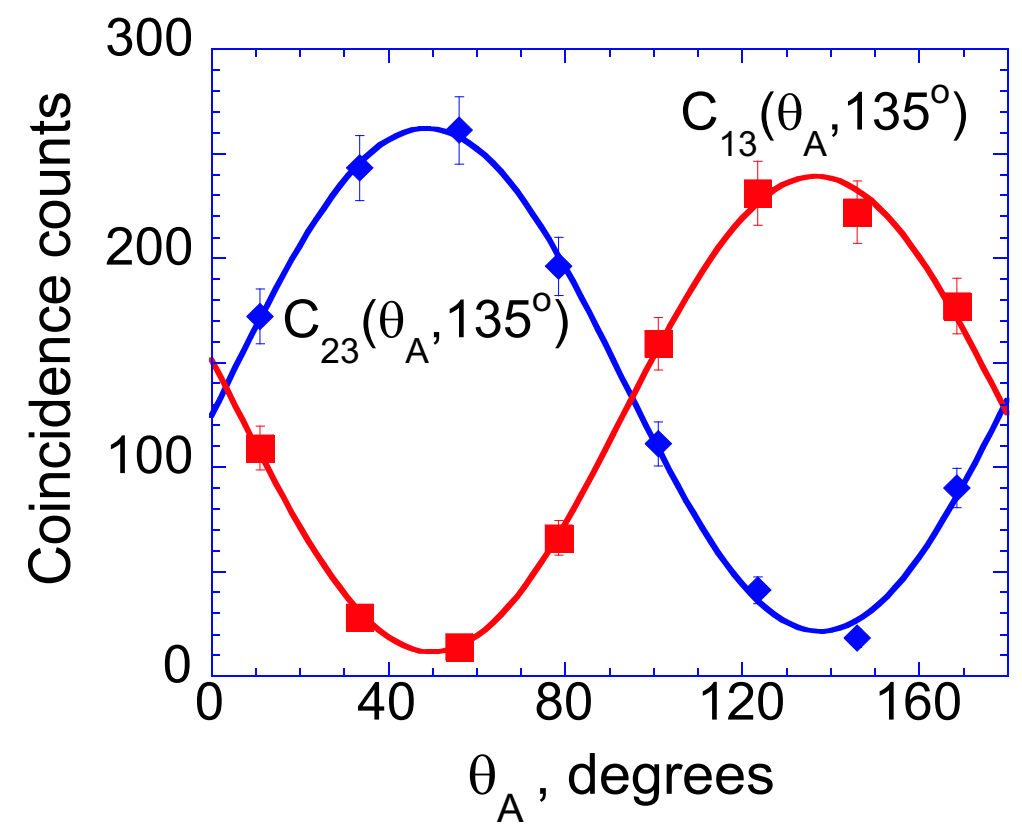

Figure 28: Measured coincidence fringes $C_{n 3}\left(\theta_{A}, \theta_{B}\right)$ as a function of $\theta_{A}$, for $\theta_{B}=135^{\circ}$, $n=1$, diamonds, $n=2$, squares. The curves are sinusoidal fits to the data. Each point is acquired for 15 minutes. The effective repetition rate is $108 \mathrm{kHz}$, each trial takes $1.1 \mu \mathrm{s}$.

beamsplitter and two single photon detectors, D1, D2 for Site $A$ and D3, D4 for Site $B$ (additional technical details are given in Refs.[46, 47, 48]). Polarization correlations between the idler fields produced at the remote sites are recorded and analyzed for the presence of entanglement. The contributions of the vacuum and single photon idler excitations are excluded in the observed photoelectric coincidences between the remote sites [109, 110]. Since quantum state transfer is a local process, it cannot generate entanglement. Hence, observation of idler field entanglement confirms probabilistic entanglement of the two remote atomic qubits. We denote the number of such coincidences between detector $D n, n=1,2$ at Site $A$ and detector $D m, m=3,4$ at Site $B$ by $C_{n m}\left(\theta_{A}, \theta_{B}\right)$. Here $\theta_{A}$ and $\theta_{B}$ are the angles by which polarization is rotated by the half-waveplates at these Sites.

The two-particle interference produces a high-visibility sinusoidal fringe pattern for the coincidence rates $C_{n m}\left(\theta_{A}, \theta_{B}\right)$, which is characteristic of entangled particles. Fig. 28 shows measured coincidence fringes for some representative angles. We calculate the coincidence rates $C_{n m}\left(\theta_{A}, \theta_{B}\right)$ to be

$$
C_{13}\left(\theta_{A}, \theta_{B}\right) \propto \epsilon_{1} \epsilon_{3} \mid\left(\cos \eta_{f}+e^{i \phi_{f}} \sin \eta_{f}\right) \sin \left(\theta_{B}+\theta_{A}\right)
$$


(a)

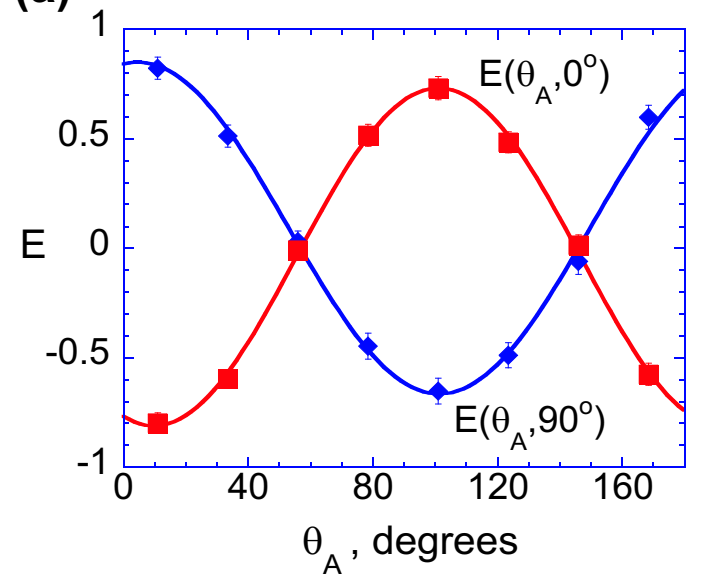

(b)

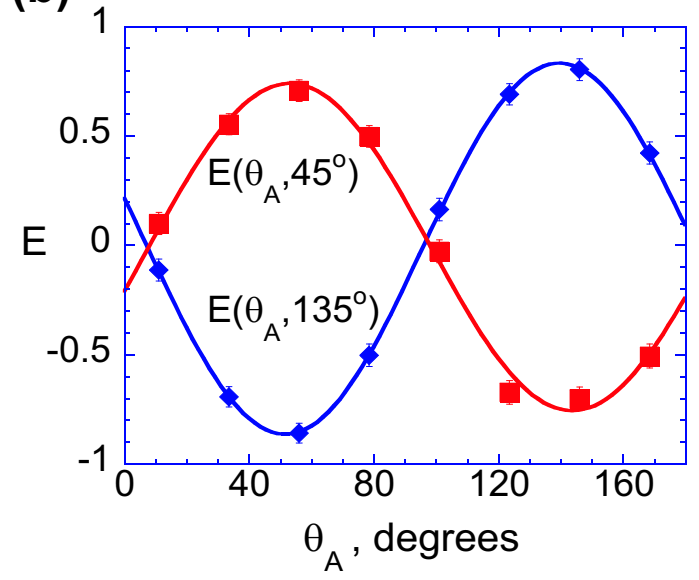

Figure 29: Measured correlation function $E\left(\theta_{A}, \theta_{B}\right)$ as a function of $\theta_{A}$. (a), $\theta_{B}=0^{\circ}$, squares, and $90^{\circ}$, diamonds. (b), $\theta_{B}=45^{\circ}$, squares, and $135^{\circ}$, diamonds. The curves are sinusoidal fits to the data.

$$
+\left.\left(\cos \eta_{f}-e^{i \phi_{f}} \sin \eta_{f}\right) \sin \left(\theta_{B}-\theta_{A}\right)\right|^{2}
$$

where $\epsilon_{m}$ is the overall efficiency (including propagation losses) for detector $D_{m}$, and similar expressions for the other three rates [59].

\subsection{Bell inequality violation}

Observation of Bell inequality violation is one method to confirm two-particle entanglement, by way of measurement of discrete values of $C_{n m}\left(\theta_{A}, \theta_{B}\right)$ at polarization settings which lie on the slopes of the fringe pattern. Explicitly, following Clauser-Horne-Shimony-Holt (CHSH) [98], we calculate the correlation function $E\left(\theta_{A}, \theta_{B}\right)$, given by

$$
\frac{C_{13}\left(\theta_{A}, \theta_{B}\right)+C_{24}\left(\theta_{A}, \theta_{B}\right)-C_{14}\left(\theta_{A}, \theta_{B}\right)-C_{23}\left(\theta_{A}, \theta_{B}\right)}{C_{13}\left(\theta_{A}, \theta_{B}\right)+C_{24}\left(\theta_{A}, \theta_{B}\right)+C_{14}\left(\theta_{A}, \theta_{B}\right)+C_{23}\left(\theta_{A}, \theta_{B}\right)} .
$$

In Fig. 22 we display $E\left(\theta_{A}, \theta_{B}\right)$ as a function of $\theta_{A}$, for four values of $\theta_{B}$. By fitting the correlation functions in Fig. 22 with sinusoids, we determine a set of four pairs of angles $\theta_{A}=78.5^{\circ}, \theta_{B}=45^{\circ}, \theta_{A}^{\prime}=33.5^{\circ}$ and $\theta_{B}^{\prime}=0^{\circ}$ that should maximize the Bell inequality violation. We acquire data for two hours at each of these four points (Table 3). In order to account for unequal efficiencies of the detectors $D 1, D 2$ and $D 3, D 4$, each correlation measurement consisted of four runs, flipping polarization of either one of the idler fields by 90 degrees between the runs. As a result, the products $\epsilon_{m} \epsilon_{n}$ are effectively replaced by 
Table 3: Measured values of the correlation function $E\left(\theta_{A}, \theta_{B}\right)$ at particular polarization settings and the Bell parameter $S$.

\begin{tabular}{ccc}
$\theta_{A}$ & $\theta_{B}$ & $E\left(\theta_{A}, \theta_{B}\right)$ \\
\hline 78.5 & 45 & $0.447 \pm 0.017$ \\
33.5 & 45 & $0.640 \pm 0.014$ \\
78.5 & 0 & $0.572 \pm 0.015$ \\
33.5 & 0 & $-0.504 \pm 0.016$ \\
& & $S=2.16 \pm 0.03$
\end{tabular}

the symmetric factor $\frac{1}{4}\left(\epsilon_{1}+\epsilon_{2}\right)\left(\epsilon_{3}+\epsilon_{4}\right)$ in Eq. (39). In this case the correlation function $E\left(\theta_{A}, \theta_{B}\right)$ becomes independent of these efficiencies:

$$
\begin{aligned}
E\left(\theta_{A}, \theta_{B}\right) & =-\frac{1}{2}\left[\cos \left(2\left(\theta_{A}-\theta_{B}\right)\right)\left(1-\cos \phi_{f} \sin 2 \eta_{f}\right)\right. \\
& \left.+\cos \left(2\left(\theta_{A}+\theta_{B}\right)\right)\left(1+\cos \phi_{f} \sin 2 \eta_{f}\right)\right]
\end{aligned}
$$

The CHSH version of the Bell inequality is then $|S| \leq 2$, where

$$
S=E\left(\theta_{A}, \theta_{B}\right)+E\left(\theta_{A}^{\prime}, \theta_{B}\right)+E\left(\theta_{A}, \theta_{B}^{\prime}\right)-E\left(\theta_{A}^{\prime}, \theta_{B}^{\prime}\right) .
$$

We find $S=2.16 \pm 0.03 \not \leq 2$, in clear violation of the Bell inequality. No corrections for background or dark counts were made to any of the experimental counting rates, and these are chiefly responsible for the reduction in the observed value of $S$ from the ideal value of 2.60 predicted by our theoretical model [59].

\subsection{Fidelity of entanglement}

An alternative method to characterize entanglement of the ensemble of detected idler-idler photoelectric correlations is to determine the fidelity with respect to the maximally entangled state $[111,103,16,109,110]$

$$
|\Psi\rangle_{M}=\left(\left|V_{A}, H_{B}\right\rangle+\left|H_{A}, V_{B}\right\rangle\right) / \sqrt{2}
$$

and this is given by

$$
\begin{aligned}
F & =\left\langle\left.\Psi\right|_{M} \rho \mid \Psi\right\rangle_{M} \\
& =\frac{1}{2}\left(\rho_{V H, V H}+\rho_{H V, H V}+\rho_{H V, V H}+\rho_{V H, H V}\right) .
\end{aligned}
$$


Table 4: Inferred density matrix elements $\rho_{i j}$. Error bars represent \pm one standard deviation and are based on the statistics of the photoelectric counting events.

\begin{tabular}{cccccc}
$\theta_{A}$ & $\theta_{B}$ & $\mathrm{HH}$ & $\mathrm{HV}$ & $\mathrm{VH}$ & $\mathrm{VV}$ \\
\hline 11 & 0 & $0.086 \pm 0.007$ & $0.315 \pm 0.012$ & $0.565 \pm 0.013$ & $0.034 \pm 0.005$ \\
56 & 45 & $0.275 \pm 0.012$ & $0.055 \pm 0.006$ & $0.060 \pm 0.006$ & $0.610 \pm 0.013$
\end{tabular}

We can write a lower bound on $F$ in terms of the diagonal matrix elements of the two-photon component of the density matrix in the original and rotated basis as follows $[16]$

$$
\begin{aligned}
F & \geq \frac{1}{2}\left(\rho_{H V, H V}+\rho_{V H, V H}-2 \sqrt{\rho_{H H, H H} \rho_{V V, V V}}\right. \\
& \left.+\bar{\rho}_{H H, H H}+\bar{\rho}_{V V, V V}-\bar{\rho}_{H V, H V}-\bar{\rho}_{V H, V H}\right) .
\end{aligned}
$$

The diagonal density matrix elements are proportional to the joint two-photon photoelectric detection probabilities, and can be expressed in terms of the coincidence counts in the original $C_{i j}\left(11^{\circ}, 0^{\circ}\right)$, and rotated, $C_{i j}\left(56^{\circ}, 45^{\circ}\right)$, bases as follows

$$
\begin{aligned}
\rho_{i j, i j} & =\frac{C_{i j}\left(11^{\circ}, 0^{\circ}\right)}{C_{13}\left(11^{\circ}, 0^{\circ}\right)+C_{23}\left(11^{\circ}, 0^{\circ}\right)+C_{14}\left(11^{\circ}, 0^{\circ}\right)+C_{24}\left(11^{\circ}, 0^{\circ}\right)} \\
\bar{\rho}_{i j, i j} & =\frac{C_{i j}\left(56^{\circ}, 45^{\circ}\right)}{C_{13}\left(56^{\circ}, 45^{\circ}\right)+C_{23}\left(56^{\circ}, 45^{\circ}\right)+C_{14}\left(56^{\circ}, 45^{\circ}\right)+C_{24}\left(56^{\circ}, 45^{\circ}\right)} .
\end{aligned}
$$

As usual, normalization by the total number of coincidences here accounts for finite measurement efficiency due to field propagation and detection losses.

Having measured each data point for one hour (Table 4), we found $F=0.77 \pm 0.01$, whereas the classical limit corresponds to $F=0.5$.

In conclusion, we have demonstrated entanglement of two remote atomic qubits, based on collective atomic states. By photoelectric detection of polarization correlations of the idler fields we have also confirmed the mapping of atomic qubit entanglement onto photonic qubits. Long-lived entanglement of remote massive qubits and entanglement transfer between matter and light are important prerequisites for realization of a scalable quantum information network. 


\section{CHAPTER VIII}

\section{QUANTUM TELECOMMUNICATION BASED ON ATOMIC CASCADE TRANSITIONS}

This chapter is based on Ref. [50].

\subsection{Motivation}

A quantum network would use the resources of distributed quantum mechanical entanglement, thus far largely untapped, for the communication and processing of information via qubits $[2,11]$. Significant advances in the generation, distribution, and storage of qubit entanglement have been made using laser manipulation of atomic ensembles, including atom-photon entanglement and matter-light qubit conversion [46], Bell inequality violation between a collective atomic qubit and a photon [47], and light-matter qubit conversion and entanglement of remote atomic qubits [101]. In each of these works photonic qubits were generated in the near-infrared spectral region. In related developments, entanglement of an ultraviolet photon with a trapped ion [16] and of a near-infrared photon with a single trapped atom $[19,18]$ have been demonstrated. Heterogeneous quantum network schemes that combine single-atom and collective atomic qubits are being actively pursued $[112,113]$. However, photons in the ultraviolet to the near-infrared range are not suited for long-distance transmission over optical fibers due to high losses.

In this Chapter, we propose a telecommunications wavelength quantum repeater based on cascade atomic transitions in either (1) a single atom or (2) an atomic ensemble. We will first discuss the latter case, with particular reference to alkali metals. Such ensembles, with long lived ground level coherences can be prepared in either solid [114] or gas [47] phase. For concreteness, we consider a cold atomic vapor confined in high-vacuum. The cascade transitions may be chosen so that the photon (signal) emitted on the upper arm has telecommunication range wavelength, while the second photon (idler), emitted to the atomic 
ground state, is naturally suited for mapping into atomic memory. Experimentally, we demonstrate phase-matched cascade emission in an ensemble of cold rubidium atoms using two different cascades: (a) at the signal wavelength $\lambda_{s}=776 \mathrm{~nm}$, via the $5 s_{1 / 2} \rightarrow 5 d_{5 / 2}$ twophoton excitation, (b) at $\lambda_{s}=1.53 \mu \mathrm{m}$, via the $5 s_{1 / 2} \rightarrow 4 d_{5 / 2}$ two-photon excitation. We observe polarization entanglement of the emitted photon pairs and superradiant temporal profiles of the idler field in both cases.

\subsection{Proposal}

We now describe our approach in detail and at the end we will summarize an alternative protocol for single atoms.

Step (A) - As illustrated in Fig. 30(a), the atomic sample is prepared in level $|a\rangle$, e.g., by means of optical pumping. It is important to note that, in the case of an atomic ensemble qubit, an incoherent mixture of Zeeman states is sufficient for our realization. The upper level $|d\rangle$, which may be of either $s$ - or $d$-type, can be excited either by one- or two-photon transitions, the latter through an intermediate level $|c\rangle$. The advantage of two photon excitation is that it allows for non-collinear phase matching of signal and idler photons; single photon excitation is forbidden in electric dipole approximation and phase-matched emission is restricted to a collinear geometry (this argument implicitly assumes that the refractive index of the vapor is approximately unity). Ideally the excitation is two-photon detuned from the upper level $|d\rangle$, creating a virtual excitation.

Step (B) - Scattering via the upper level $|d\rangle$ to ground level $|a\rangle$ through the intermediate level $|e\rangle$ (where $|e\rangle$ may coincide with $|c\rangle$ ) results in the cascaded emission of signal and idler fields. The signal field, which is emitted on the upper arm, has a temporal profile identical to that of the laser excitation as a consequence of the large two photon detuning. As noted earlier, the wavelength of this field lies in the 1.1-1.6 $\mu \mathrm{m}$ range, depending on the alkali metal transition used. The signal field can be coupled to an optical fiber (which may have losses as low as $0.2 \mathrm{~dB} / \mathrm{km}$ ) and transmitted to a remote location.

The temporal profile of the idler field can be much shorter than the single-atom spontaneous decay time $t_{s}$ of the intermediate level. Under the conditions of a large Fresnel 


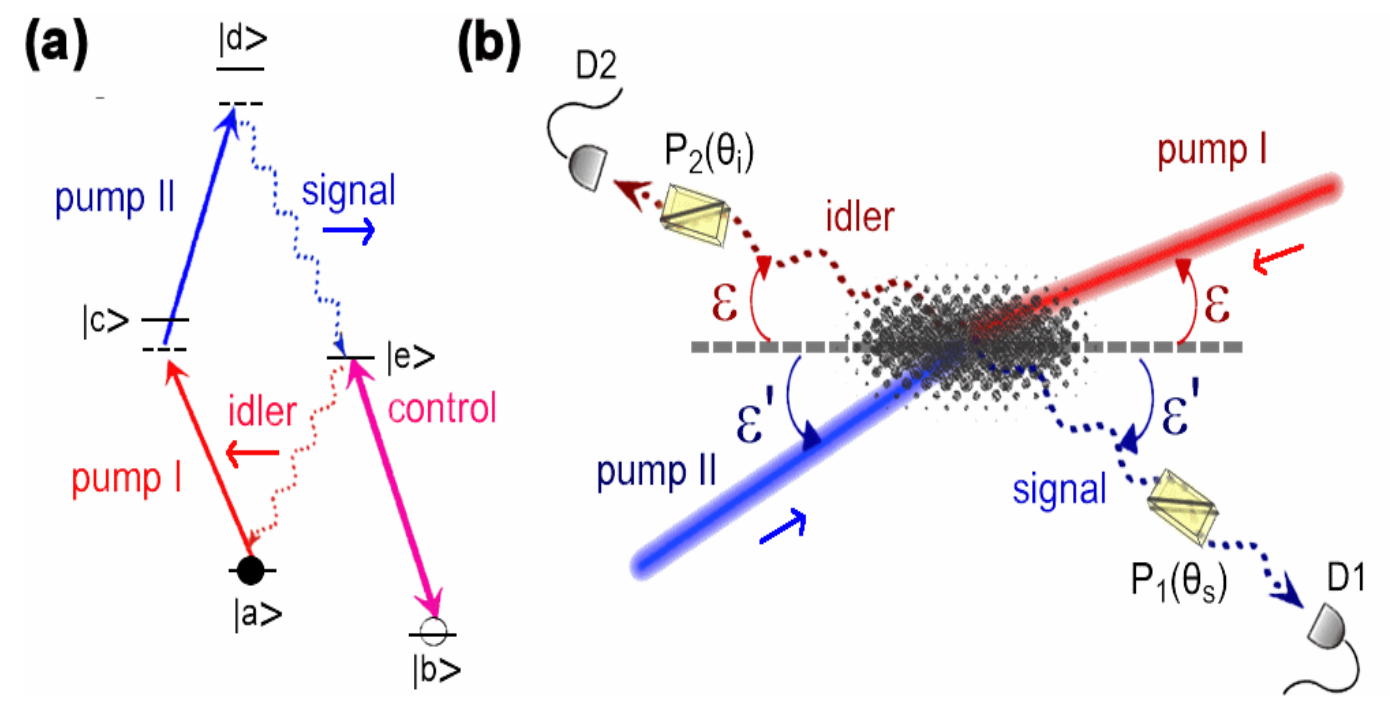

Figure 30: (a) The atomic structure for the proposed cascade emission scheme involving excitation by pumps I and II. Pump II and the signal photons lie in the telecommunication wavelength range when a suitable level of orbital angular momentum $L=0$ or $L=2$ is used as level $|d\rangle$. For atomic rubidium, the signal wavelength is $1.32 \mu \mathrm{m}\left(6 s_{1 / 2} \rightarrow 5 p_{1 / 2}\right.$ transition), $1.37 \mu \mathrm{m}\left(6 s_{1 / 2} \rightarrow 5 p_{3 / 2}\right.$ transition), $1.48 \mu \mathrm{m}\left(4 d_{3 / 2(5 / 2)} \rightarrow 5 p_{1 / 2}\right.$ transition), $1.53 \mu \mathrm{m}\left(4 d_{3 / 2(5 / 2)} \rightarrow 5 p_{3 / 2}\right.$ transition). For atomic cesium, the signal wavelength is 1.36 $\mu \mathrm{m}\left(7 s_{1 / 2} \rightarrow 6 p_{1 / 2}\right.$ transition), $1.47 \mu \mathrm{m}\left(7 s_{1 / 2} \rightarrow 6 p_{3 / 2}\right.$ transition $)$. For $\mathrm{Na}$ and $\mathrm{K}$ the corresponding wavelengths are in the 1.1-1.4 $\mu \mathrm{m}$ range. (b) Schematic of experimental setup based on ultra-cold ${ }^{85} \mathrm{Rb}$ atomic gas. For $\lambda_{s}=776 \mathrm{~nm}$, phase matching results in the angles $\varepsilon^{\prime} \approx \varepsilon \approx 1^{\circ}$, while for $\lambda_{s}=1.53 \mu \mathrm{m}, \varepsilon^{\prime} \approx 2 \varepsilon \approx 2^{\circ}$. $P_{1}$ and $P_{2}$ are polarizers; D1 and D2 are detectors.

number of the exciting laser fields, the decay time is of order $t_{s} / d_{t h}$, characteristic of superradiance $[115,116,30]$. Here $d_{t h} \approx 3 n \lambda^{2} l /(8 \pi)$ is the optical thickness, where $\lambda$ is the wavelength, $n$ is the number density and $l$ is the length of the sample.

The direction of the idler field is determined by the phase matching condition $\vec{k}_{1}+\vec{k}_{2}=$ $\vec{k}_{s}+\vec{k}_{i}$, where $\vec{k}_{1}$ and $\vec{k}_{2}$ are the wavevectors of the laser fields I and II, respectively. Under conditions of phase matching, collective enhancement causes emission of the the idler photon correlated with a return of the atom into the Zeeman state from which it originated [47]. The fact that the atom begins and ends the absorption-emission cycle in the same state is essential for strong signal-idler polarization correlations. The reduced density operator for the field, taking into account collective enhancement, was derived in Ref. [59]:

$$
\hat{\rho}(t) \approx\left(1+\sqrt{\epsilon} \hat{A}_{2}^{\dagger}(t)\right) \hat{\rho}_{v a c}\left(1+\sqrt{\epsilon} \hat{A}_{2}(t)\right)
$$


where $\hat{\rho}_{v a c}$ is the vacuum state of the field, $\hat{A}_{2}^{\dagger}(t)$ is a time dependent two photon creation operator for the signal and idler fields, and $\epsilon \ll 1$. For linearly polarized pumps with parallel (vertical) polarizations, we find

$$
\hat{A}_{2}^{\dagger}(t)=\cos \chi \hat{a}_{H}^{\dagger} \hat{b}_{H}^{\dagger}+\sin \chi \hat{a}_{V}^{\dagger} \hat{b}_{V}^{\dagger}
$$

where $\chi$ is determined by Clebsch-Gordan coupling coefficients [59], $\hat{a}_{H(V)}^{\dagger}$ and $\hat{b}_{H(V)}^{\dagger}$ are creation operators for a horizontally (vertically) polarized signal and idler photon, respectively. For the hyperfine level configuration $F_{a}=3 \rightarrow F_{c}=4=F_{e} \rightarrow F_{d}=5$, and for an unpolarized atomic sample, we find $\sin \chi=2 \cos \chi=2 / \sqrt{5}$.

Step $(C)$ - The photonic qubit is encoded in the idler field polarization. Photonic to atomic qubit conversion was achieved in Ref.[101]. Such conversion can be performed either within the same ensemble or in a suitably prepared adjacent ensemble/pair of ensembles. In either case, a strong laser control beam is required to couple the other ground hyperfine level $|b\rangle$ to the intermediate level $|e\rangle$. Collective excitations involving two orthogonal hyperfine coherences serve as the logical states of the atomic qubit [46, 47, 101].

\subsection{Experiment}

We observe phase-matched cascade emission of entangled photon pairs, using samples of cold ${ }^{85} \mathrm{Rb}$ atoms, for two different atomic cascades: (a) at $\lambda_{s}=776 \mathrm{~nm}$, via the $5 s_{1 / 2} \rightarrow 5 d_{5 / 2}$ two-photon excitation, (b) at $\lambda_{s}=1.53 \mu \mathrm{m}$, via the $5 s_{1 / 2} \rightarrow 4 d_{5 / 2}$ two-photon excitation. The investigations are carried out in two different laboratories using similar setups, Fig. 30(b). A magneto-optical trap (MOT) of ${ }^{85} \mathrm{Rb}$ provides an optically thick cold atomic cloud. The atoms are prepared in an incoherent mixture of the level $|a\rangle$, which corresponds to the $5 s_{1 / 2}, F_{a}=3$ ground level, by means of optical pumping. The intermediate level $|c\rangle=|e\rangle$ corresponds to the $5 p_{3 / 2}, F_{c}=4$ level of the $D_{2}$ line at $780 \mathrm{~nm}$, and the excited level $|d\rangle$ represents (a) the $5 d_{5 / 2}$ level with $\lambda_{s}=776 \mathrm{~nm}$, or (b) the $4 d_{5 / 2}$ level with $\lambda_{s}=1.53$ $\mu \mathrm{m}$. Atomic level $|b\rangle$ corresponds to $5 s_{1 / 2}, F_{b}=2$, and could be used to implement the light-to-matter qubit conversion [101].

The trapping and cooling light as well as the quadrupole magnetic field of the MOT are switched off for the $2 \mathrm{~ms}$ duration of the measurement. The ambient magnetic field is 
compensated by three pairs of Helmholtz coils. Counterpropagating pumps I (at $780 \mathrm{~nm}$ ) and II (at $776 \mathrm{~nm}$ or $1.53 \mu \mathrm{m}$ ), tuned to two-photon resonance for the $|a\rangle \rightarrow|d\rangle$ transition are focused into the MOT using the off-axis, counter-propagating geometry of Harris and coworkers [54]. This two-photon excitation induces phase-matched signal and idler emission.

With quasi-cw pump fields, we perform photoelectric coincidence detection of the signal and idler fields. The latter are directed onto single photon detectors D1 and D2. For $\lambda_{s}=1.53 \mu \mathrm{m}$, the signal field is coupled into $100 \mathrm{~m}$ of single-mode fiber, and detector D1 (cooled InGaAs photon counting module) is gated using the output pulse of silicon detector D2. The electronic pulses from the detectors are fed into a time-interval analyzer with $1 \mathrm{~ns}$ time resolution.

We measure the stationary signal-idler intensity correlation function $G_{s i}(\tau)=\langle\mathcal{T}$ : $\left.\hat{I}_{s}(t) \hat{I}_{i}(t+\tau):\right\rangle$, where the notation $\mathcal{T}::$ denotes time and normal ordering of operators, and $\hat{I}_{s}$ and $\hat{I}_{i}$ are the signal and idler intensity operators, respectively [30]. Results for (a) $\lambda_{s}=776 \mathrm{~nm}$ and (b) $\lambda_{s}=1.53 \mu \mathrm{m}$ are presented in Fig. 31 and Fig. 32, respectively. In particular, the measured correlation functions are shown in Fig. 31(a,b) and Fig. 32(a). The correlation function shown in Fig. 31(a) exhibits quantum beats due to the two different hyperfine components of the the $5 p_{3 / 2}$ level [117]. The correlation times are consistent with superradiant scaling $\sim t_{s} / d_{t h}$, Fig. $31(\mathrm{c})$, where $t_{s} \approx 27$ ns for the $5 p_{3 / 2}$ level $[115,116]$.

In order to investigate polarization correlations of the signal and idler fields, they are passed through polarizers $P_{1}$ (set at angle $\theta_{s}$ ) and $P_{2}$ (set at angle $\theta_{i}$ ), respectively, as shown in Fig. 30(b). We integrate the time-resolved counting rate over a window $\Delta T$ centered at the maximum of the signal-idler intensity correlation function $G_{s i}(\tau)$, with (a) $\Delta T=6$ ns for $\lambda_{s}=776 \mathrm{~nm}$, and (b) $\Delta T=1 \mathrm{~ns}$ for $\lambda_{s}=1.53 \mu \mathrm{m}$. The resulting signal-idler coincidence rate $C\left(\theta_{s}, \theta_{i}\right)$ exhibits sinusoidal variation as a function of the polarizers' orientations, as shown in Figs. 31(d) and 32(b). In order to verify the predicted polarization entanglement, we check for violation of Bell's inequality $S \leq 2[98,30,75]$. We first calculate the correlation function $E\left(\theta_{s}, \theta_{i}\right)$, given by

$$
\frac{C\left(\theta_{s}, \theta_{i}\right)+C\left(\theta_{s}^{\perp}, \theta_{i}^{\perp}\right)-C\left(\theta_{s}^{\perp}, \theta_{i}\right)-C\left(\theta_{s}, \theta_{i}^{\perp}\right)}{C\left(\theta_{s}, \theta_{i}\right)+C\left(\theta_{s}^{\perp}, \theta_{i}^{\perp}\right)+C\left(\theta_{s}^{\perp}, \theta_{i}\right)+C\left(\theta_{s}, \theta_{i}^{\perp}\right)},
$$



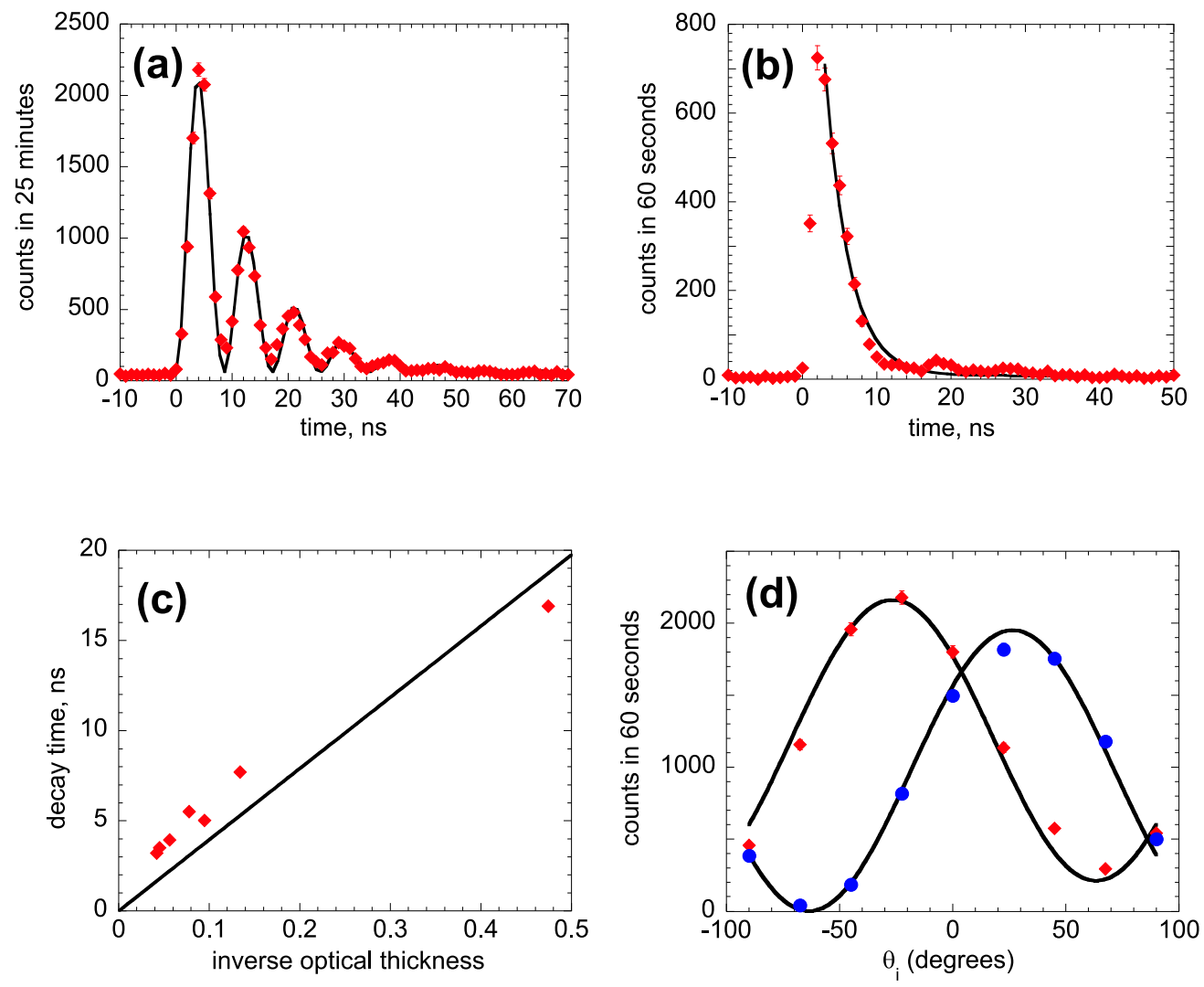

Figure 31: (a) Count rate proportional to the signal-idler intensity correlation function $G_{s i}$ as a function of signal-idler delay $\tau,|d\rangle=\left|5 d_{5 / 2}, F=4\right\rangle$. The quantum beats are associated with $120 \mathrm{MHz}$ hyperfine splitting, $F=3$ and 4 , of the $5 p_{3 / 2}$ level. The solid curve is a fit of the form $\beta+A \exp (-t / \alpha) \sin ^{2}(\pi \Omega t)$, where $\beta=63, A=2972, \alpha=11$ ns and $\Omega=117$ $\mathrm{MHz}$ are adjustable parameters. (b) Same as (a), but for $|d\rangle=\left|5 d_{5 / 2}, F=5\right\rangle$. Since this state can only decay though the $F=4$ component of the $5 p_{3 / 2}$ level, there are no quantum beats. The solid curve is an exponential fit with decay time of $3.2 \mathrm{~ns}$. (c) The measured decay time vs the inverse measured optical thickness. (d) Measured coincidence fringes for $\theta_{s}=45^{\circ}$ (red diamonds) and $\theta_{s}=135^{\circ}$ (blue circles). The solid curves are fits based on Eqs. $(45,46)$, with $\cos \chi=1 / \sqrt{5}$. 

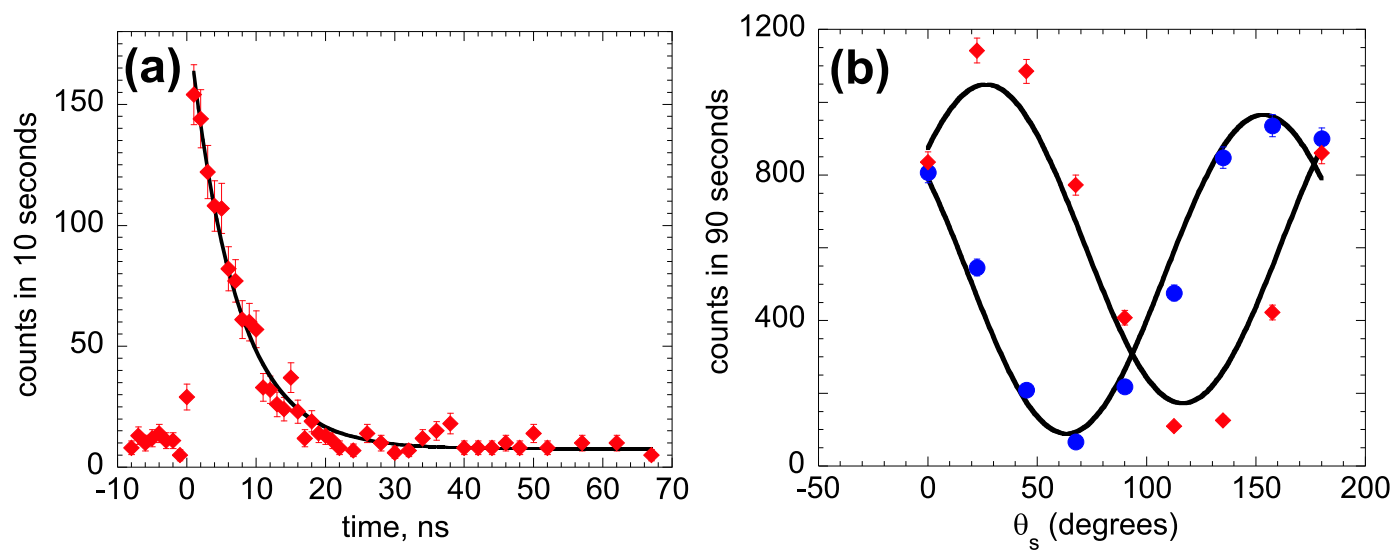

Figure 32: (a) Same as Fig. 31(a,b), but for $|d\rangle=\left|4 d_{5 / 2}, F=5\right\rangle$. The solid curve is an exponential fit with decay time of $6.7 \mathrm{~ns}$. (b) Measured coincidence fringes for $\theta_{i}=45^{\circ}$ (red diamonds) and $\theta_{i}=135^{\circ}$ (blue circles). The solid curves are fits based on Eqs. $(45,46)$, with $\cos \chi=1 / \sqrt{5}$.

Table 5: Measured correlation function $E\left(\theta_{s}, \theta_{i}\right)$ and $S$ for $\lambda_{s}=776 \mathrm{~nm}$ and $\lambda_{s}=1.53 \mu \mathrm{m}$.

\begin{tabular}{cccc}
$\lambda_{s}$ & $\theta_{s}$ & $\theta_{i}$ & $E\left(\theta_{s}, \theta_{i}\right)$ \\
\hline & $0^{\circ}$ & $-67.5^{\circ}$ & $-0.670 \pm 0.011$ \\
$776 \mathrm{~nm}$ & $45^{\circ}$ & $-22.5^{\circ}$ & $-0.503 \pm 0.013$ \\
& $0^{\circ}$ & $-22.5^{\circ}$ & $0.577 \pm 0.012$ \\
& $45^{\circ}$ & $-67.5^{\circ}$ & $-0.434 \pm 0.014$ \\
& & & $S=2.185 \pm 0.025$ \\
\hline \multirow{4}{*}{$1.53 \mu \mathrm{m}$} & $22.5^{\circ}$ & $45^{\circ}$ & $-0.554 \pm 0.027$ \\
& $67.5^{\circ}$ & $0^{\circ}$ & $-0.682 \pm 0.027$ \\
& $22.5^{\circ}$ & $0^{\circ}$ & $0.473 \pm 0.024$ \\
& $67.5^{\circ}$ & $45^{\circ}$ & $-0.423 \pm 0.029$ \\
& & & $S=2.132 \pm 0.036$
\end{tabular}

where $\theta^{\perp}=\theta+\pi / 2$, and $S=\left|E\left(\theta_{s}, \theta_{i}\right)+E\left(\theta_{s}{ }^{\prime}, \theta_{i}\right)\right|+\left|E\left(\theta_{s}, \theta_{i}^{\prime}\right)-E\left(\theta_{s}^{\prime}, \theta_{i}^{\prime}\right)\right|$.

Measured values of $E\left(\theta_{s}, \theta_{i}\right)$, using the set of angles $\theta_{s}, \theta_{i}$, chosen to maximize the violation of Bell's inequality, are presented in Table 5. We find (a) $S=2.185 \pm 0.025$ for $\lambda_{s}=776 \mathrm{~nm}$, and (b) $S=2.132 \pm 0.036$ for $\lambda_{s}=1.53 \mu \mathrm{m}$, consistent with polarization entanglement of signal and idler fields in both cases. The entangled two-photon state of Eqs. $(45,46)$, for $\sin \chi=2 / \sqrt{5}$, has a substantial degree of asymmetry. If oppositely, circularly, polarized pumps I and II were used, the corresponding two-photon state would be symmetric with $\sin \chi=\cos \chi=1 / \sqrt{2}[59]$. 


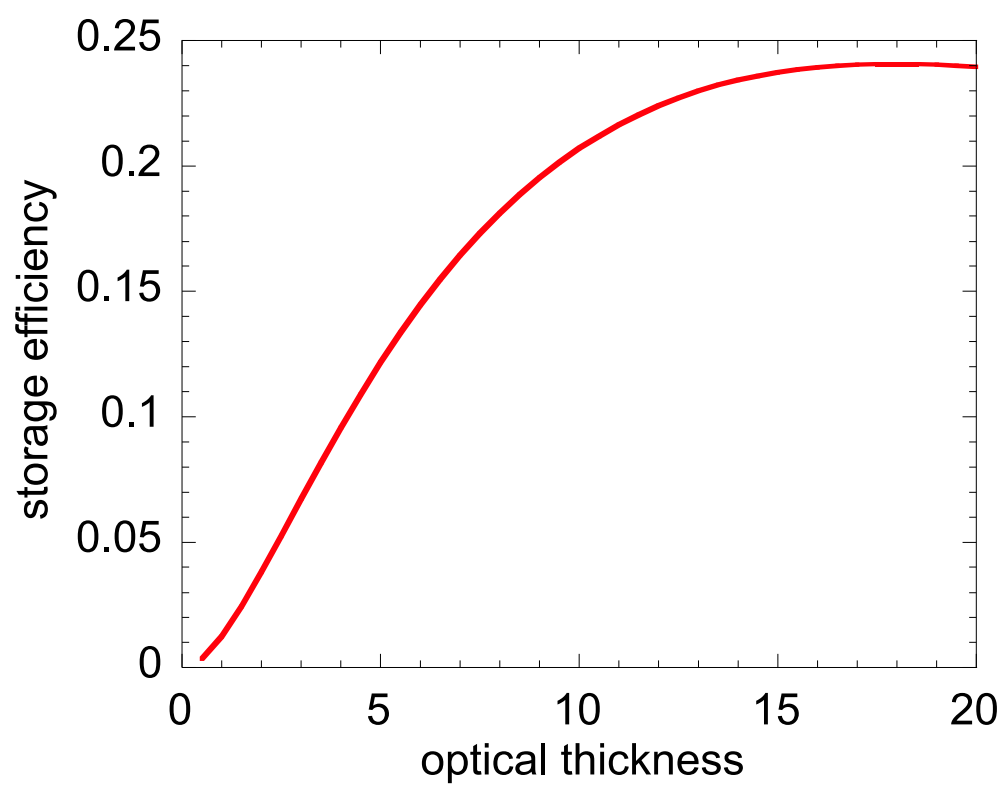

Figure 33: Efficiency of storage and subsequent retrieval of a coherent idler field with decay time of $6 \mathrm{~ns}$ in an auxiliary atomic ensemble, obtained by numerical integration of the Maxwell-Bloch equations. The atomic coherence time is assumed to be much longer than the storage time.

The quantum repeater protocol involves sequential entanglement swapping via Hong-OuMandel (HOM) interference followed by coincidence detection [30, 11]. High-visibility HOM interference requires that the signal and idler photon wave-packets have no entanglement in the time or frequency domains $[118,119,120]$. This may be achieved with excitation pulses that are far detuned from two-photon resonance and with pulse lengths much shorter than the superradiant emission time $t_{s} / d_{t h}$ of level $|e\rangle$.

The idler field qubit is naturally suited for conversion into an atomic qubit encoded into the collective hyperfine coherence of levels $|a\rangle=\left|5 s_{1 / 2}, F=3\right\rangle$ and $|b\rangle=\left|5 s_{1 / 2}, F=2\right\rangle$. To perform such conversion, either the same or another similar ensemble/pair of ensembles could be employed [101], (see also Chapter 4). A time-dependent control laser field resonant on the $|b\rangle=\left|5 s_{1 / 2}, F=2\right\rangle \leftrightarrow|e\rangle=\left|5 p_{3 / 2}, F=3\right\rangle$ transition could selectively convert one of the two frequency components of the idler field, shown in Fig. 31(a), into a collective atomic qubit. Pulsed excitation should be used in order to enable the synchronization of the idler qubit and the control laser. Numerical simulations show that qubit conversion and subsequent retrieval can be done with good efficiency for moderate optical thicknesses (Fig. 33), even though the idler field temporal profile is shorter than those employed in Ref. 
[101] (compare with Fig. 6).

The basic protocols we have outlined can also be applied to single alkali atom emitters. Similar cascade decays in single atoms were used in early experiments demonstrating violation of local realism [121] and single photon generation [65]. For alkali metal atoms, it is necessary to optically pump the atom into a single Zeeman state, e.g., $m=0$, of level $|a\rangle$. A virtual excitation of a single Zeeman state of level $|d\rangle$ is created with short laser pulses. Coherent Raman scattering to level $|e\rangle$ results in atom-photon polarization entanglement. In order to prevent spontaneous decay of the level $|e\rangle$, a control field $\pi$-pulse is applied immediately after the application of the two-photon excitation, transferring the atomic qubit into the ground state where it could live for a long time. It is important that the $\pi$-pulse duration is shorter than the spontaneous lifetime of level $|e\rangle$. Two-photon interference and photoelectric detection of signal photons produced by two remote single atom nodes would result in entanglement of these remote atomic qubits [104]. Qubit detection for single atoms can be achieved with nearly unit efficiency and in a time as short as $50 \mu$ s $[16,17]$. Such high efficiency and speed lead to the possibility of a loophole-free test of Bell's inequality, for atoms separated by about 30 kilometers. Cascaded entanglement swapping between successive pairs of remote entangled atomic qubits may be achieved via local coupling of one of the atoms from the first pair and its neighboring partner from the the following pair $[12]$.

We also point out that the cascade level scheme employed here can be used to convert a telecommunications photon into a near-infrared photon using four-wave mixing. This could potentially be useful because single-photon detectors for the visible and near-infrared currently have much higher quantum efficiency, and much lower dark count probability, than practically viable (e.g., InGaAs) detectors used at telecommunication wavelengths. 


\section{CHAPTER IX}

\section{CONCLUSION}

Quantum information processing using atomic ensembles is a fast growing field. In the past few years significant progress has been made to turn this paradigm into a practically viable quantum network systems.

The generation of photon pairs using atomic ensembles has been observed in ensembles of cold atoms both in free space [43, 45] and in a cavity [55], as well as using a hot atomic vapor [44]. The quantum state transfer between atomic ensembles and a single photon and atom-photon entanglement have been demonstrated [46]. This breakthrough laid the groundwork for further advances, such as the demonstration of Bell inequality violation between a collective atomic qubit and a photon [47], storage and retrieval of single photons [48], collapses and revivals of quantum memory [60, 49], light-matter qubit conversion, and entanglement of remote atomic qubits [101]. A practical scheme to achieve long-distance quantum communication over optical fibers, involving cascade atomic transitions was proposed, and its critical elements are verified experimentally [50].

To illustrate the extent of the progress, one could note that the signal-idler correlation function $g_{s i}-2=0.46$ reported in the first experiment [43] has been since improved by three orders of magnitude in Refs. [101, 48]. As another example, Fig. 34 shows the coherence time of atomic memory based on atomic ensemble as a function of experiment date $[46,47,48,76]$.

However these advances are still not sufficient for the realization of the long distance quantum repeater. For example in order to build a repeater that operates over 1000 kilometers one needs coherence times in excess of $100 \mathrm{~ms}$. It is three orders of magnitude higher than the best result achieved so far [76].

The main problem here is that the atomic spin wave that stores quantum information in atomic ensemble is very sensitive to the external magnetic field. Most experiments were 


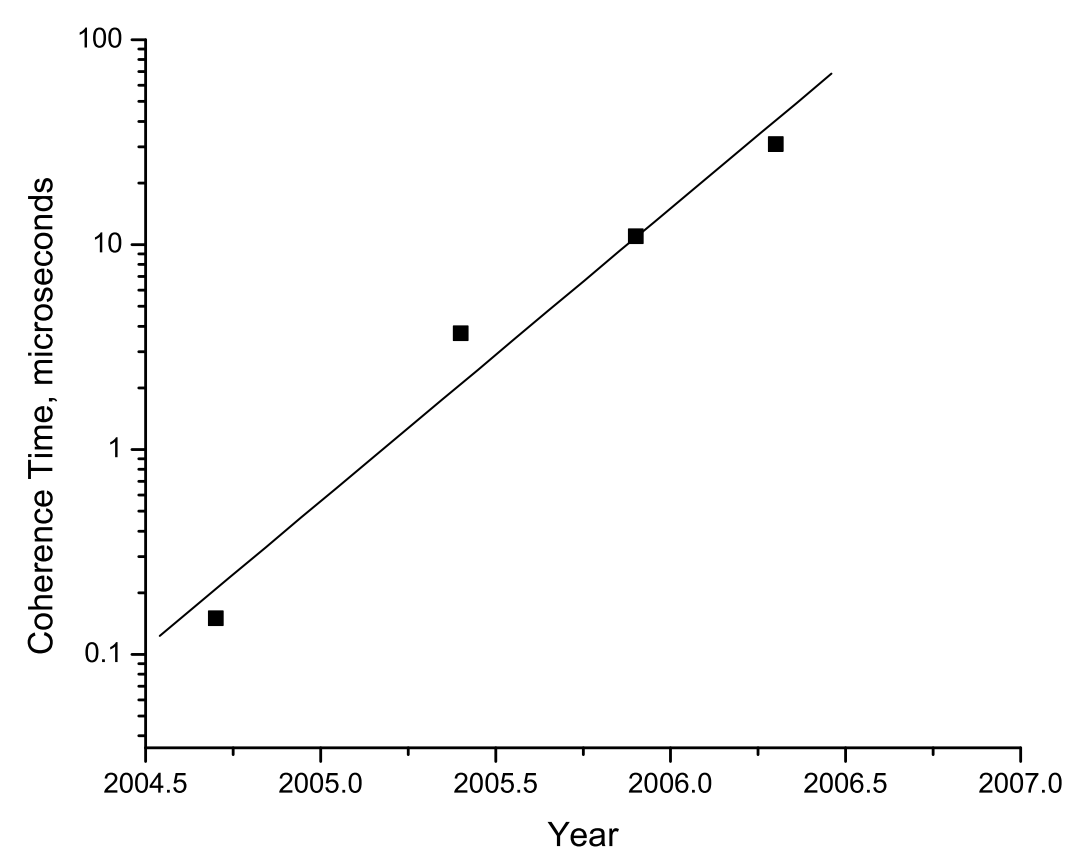

Figure 34: Coherence time as a function of experiment date

performed using magneto-optical traps, where stray magnetic field is difficult to avoid. Another source of decoherence is the motion of the atoms within the sample. It eventually washes out the spin wave and limits the coherence time to $70-700 \mu$ s depending on the beam geometry.

A possible solution is to use an atomic ensemble confined in an optical dipole trap. As the dipole trap does not involve any magnetic field, it can be better isolated from the ambient influence. Moreover, motion of atoms in the optical dipole trap can be reduced by using an optical lattice geometry. In order to reduce sensitivity to the stray magnetic field one may also employ a magnetically insensitive transition $m=0 \rightarrow m^{\prime}=0$.

Of course, a lot of work remains to be done in order to integrate all of the demonstrated components into a single practical system. Nevertheless, the results reported in this thesis suggest that the goal of building the quantum repeater for the long distance communication is within the reach of current technology. 


\section{REFERENCES}

[1] I. Chuang and M. Nielsen, Quantum computation and quantum information (Cambridge University Press, 2000).

[2] H.-J. Briegel, W. Dur, J. I. Cirac, and P. Zoller, "Quantum repeaters: The role of imperfect local operations in quantum communication," Phys. Rev. Lett. 81, 5932 (1998).

[3] A. K. Ekert, "Quantum cryptography based on Bell theorem," Phys. Rev. Lett. 67, 661 (1991).

[4] C. H. Bennett and G. Brassard, in Proceedings of the international conference on computers, systems and signal processing Indian Institute of Science, Bangalore, India 1984.

[5] C. H. Bennett, G. Brassard, C. Crepeau, R. Jozsa, A. Peres, and W. K. Wootters, "Quantum cryptography based on bell theorem," Phys. Rev. Lett. 70, 895 (1993).

[6] D. Bouwmeester, J. W. Pan, M. Mattle, K.and Eibl, H. Weinfurter, and A. Zeilinger, "Experimental quantum teleportation," Nature 390, 575 (1997).

[7] D. Boschi, S. Branca, F. D. Martini, L. Hardy, and S. Popescu, "Experimental realization of teleporting an unknown pure quantum state via dual classical and einsteinpodolsky-rosen channels," Phys. Rev. Lett. 80, 1121 (1998).

[8] N. A. Peters, J. T. Barreiro, M. E. Goggin, T.-C. Wei, and P. G. Kwiat, "Remote state preparation: arbitrary remote control of photon polarization," Phys. Rev. Lett. 94, 150502 (2005).

[9] C. Gobby, Z. L. Yuan, and A. J. Shields, "Quantum key distribution over $122 \mathrm{~km}$ of standard telecom fiber," Appl. Phys. Lett. 84, 3762 (2004).

[10] M. Aspelmeyer, T. Jennewein, M. Pfennigbauer, W. R. Leeb, and A. Zeilinger, "Longdistance quantum communication with entangled photons using satellites," IEEE Journal of Selected Topics in Quantum Electronics 9, 1541 (2003).

[11] L.-M. Duan, M. D. Lukin, I. J. Cirac, and P. Zoller, "Long-distance quantum communication with atomic ensembles and linear optics," Nature 414, 413 (2001).

[12] L. M. Duan, B. B. Blinov, D. L. Moehring, and C. Monroe, "Scalable trapped ion quantum computation with a probabilistic ion-photon mapping," Quant. Inf. and Comp. 4, 165 (2004).

[13] S. Lloyd, M. S. Shahriar, J. H. Shapiro, and P. R. Hemmer, "Long distance, unconditional teleportation of atomic states via Complete Bell State Measurements," Phys. Rev. Lett. 87, 167903 (2001). 
[14] L. Childress, J. M. Taylor, A. S. Sorensen, and M. D. Lukin, "Fault-tolerant quantum communication based on solid-state photon emitters," Phys. Rev. Lett. 96, 070504 (2006).

[15] E. Hagley, X. Maitre, G. Nogues, C. Wunderlich, M. Brune, J. M. Raimond, and S. Haroche, "Generation of Einstein-Pololsky-Rosen pairs of atoms," Phys. Rev. Lett. 79, 1 (1997).

[16] B. B. Blinov, D. L. Moehring, L.-M. Duan, and C. Monroe, "Observation of entanglement between a single trapped atom and a single photon," Nature 428, 153 (2004).

[17] D. L. Moehring, M. J. Madsen, B. B. Blinov, and C. Monroe, "Experimental Bell inequality violation with an atom and a photon," Phys. Rev. Lett. 93, 090410 (2004).

[18] J. Volz, M. Weber, D. Schlenk, W. Rosenfeld, J. Vrana, K. Saucke, C. Kurtsiefer, and H. Weinfurter, "Observation of entanglement of a single photon with a trapped atom," Phys. Rev. Lett. 96, 030404 (2006).

[19] M. Weber, Quantum optical experiments towards atom-photon entanglement, $\mathrm{PhD}$ thesis University of Munich 2005.

[20] A. Kuhn, M. Hennrich, and G. Rempe, "Deterministic single-photon source for distributed quantum networking," Phys. Rev. Lett. 89, 67901 (2002).

[21] J. McKeever, A. Boca, A. D. Boozer, R. Miller, J. R. Buck, A. Kuzmich, and H. J. Kimble, "Deterministic generation of single photons from one atom trapped in a cavity," Science 303, 1992 (2004).

[22] M. Lukin, "Colloquium: Trapping and manipulating photon states in atomic ensembles," Rev. Mod. Phys. 75, 457 (2003).

[23] A. Kuzmich and E. S. Polzik, "Atomic continuous variables processing and light-atoms quantum interface,", in Quantum information with continuous variables, edited by S. L. Braunstein and A. K. Pati Kluwer 2003.

[24] M. Bajcsy, A. Zibrov, and M. D. Lukin, "Stationary pulses of light in an atomic medium," Nature 426, 638 (2003).

[25] A. S. Zibrov, A. B. Matsko, O. Kocharovskaya, Y. V. Rostovtsev, G. R. Welch, and M. O. Scully, "Transporting and time reversing light via atomic coherence," Phys. Rev. Lett. 88, 103601 (2002).

[26] D. F. Phillips, A. Fleischhauer, A. Mair, R. L. Walsworth, and M. D. Lukin, "Storage of light in atomic vapor," Phys. Rev. Lett. 86, 783 (2001).

[27] C. Liu, Z. Dutton, C. H. Behroozi, and L. V. Hau, "Observation of coherent optical information storage in an atomic medium using halted light pulses," Nature 409, 490 (2001).

[28] M. Johnsson and K. Molmer, "Storing quantum information in a solid using dark-state polaritons," Phys. Rev. A. 70, 032320 (2004). 
[29] R. H. Dicke, "Coherence in spontaneous radiation processes," Phys. Rev. 93, 99 (1954).

[30] L. Mandel and E. Wolf, Optical Coherence and Quantum Optics (Cambridge University Press, 1995).

[31] A. Kuzmich, K. Molmer, and E. S. Polzik, "Spin squeezing in an ensemble of atoms illuminated with squeezed light," Phys. Rev. Lett. 79, 4782 (1997).

[32] R. Molmer, "Twin-correlations in atoms," Eur. Phys. J. D 5, 301 (1999).

[33] G. Smith, S. Chaudhury, and P. Jessen, "Faraday spectroscopy in an optical lattice: a continuous probe of atom dynamics," J.Opt. B: Quant. Semiclass. Opt. 5, 323 (2003).

[34] G. Grom and A. M. Kuzmich, "Generation of spin squeezed states for subquantum interferometry," JETP Lett. 61, 900 (1995).

[35] A. Kuzmich, N. P. Bigelow, and L. Mandel, "Atomic quantum non-demolition measurements and squeezing," Europhys. Lett. 42, 481 (1998).

[36] A. Kuzmich, L. Mandel, J. Janis, Y. E. Young, R. Ejnisman, and N. P. Bigelow, "Quantum nondemolition measurements of collective atomic spin," Phys. Rev. A 60, 2346 (1999).

[37] A. Kuzmich, L. Mandel, and N. P. Bigelow, "Generation of spin squeezing via continuous quantum nondemolition measurement," Phys. Rev. Lett. 85, 1594 (2000).

[38] A. Kuzmich and E. S. Polzik, "Atomic quantum state teleportation and swapping," Phys. Rev. Lett. 85, 5639 (2000).

[39] B. Julsgaard, J. Sherson, J. I. Cirac, J. Fiurasek, and E. S. Polzik, "Experimental demonstration of quantum memory for light," Nature 432, 482 (2004).

[40] S. E. Harris, "Electromagnetically induced transparency," Phys. Today 50, 36 (1997).

[41] L. V. Hau, S. E. Harris, Z. Dutton, and C. H. Behroozi, "Light speed reduction to 17 metres per second in an ultracold atomic gas," Nature 397, 594 (1999).

[42] M. Fleischhauer and M. D. Lukin, "Dark-state polaritons in electromagnetically induced transparency," Phys. Rev. Lett. 84, 5094 (2000).

[43] A. Kuzmich, W. P. Bowen, A. D. Boozer, A. Boca, C. W. Chou, L.-M. Duan, and H. J. Kimble, "Generation of nonclassical photon pairs for scalable quantum communication with atomic ensembles," Nature 423, 731 (2003).

[44] C. H. van der Wal, M. D. Eisaman, A. Andre, R. L. Walsworth, D. F. Phillips, A. S. Zibrov, and M. D. Lukin, "Atomic memory for correlated photon states," Science 301 (2003).

[45] C. Chou, S. Polyakov, A. Kuzmich, and H. Kimble, "Single-photon generation from stored excitation in an atomic ensemble," Phys. Rev. Lett. 92, 213601 (2004).

[46] D. N. Matsukevich and A. Kuzmich, "Quantum state transfer between matter and light," Science 306, 663 (2004). 
[47] D. N. Matsukevich, T. Chanelière, M. Bhattacharya, S. Y. Lan, S. D. Jenkins, T. A. B. Kennedy, and A. Kuzmich, "Entanglement of a photon and a collective atomic excitation," Phys. Rev. Lett. 95, 040405 (2005).

[48] T. Chanelière, D. N. Matsukevich, S. D. Jenkins, S. Y. Lan, T. A. B. Kennedy, and A. Kuzmich, "Storage and retrieval of single photons transmitted between remote quantum memories," Nature 438, 833 (2005).

[49] D. N. Matsukevich, T. Chanelière, S. D. Jenkins, S. Y. Lan, T. A. B. Kennedy, and A. Kuzmich, "Observation of dark state polariton collapses and revivals," Phys. Rev. Lett. 96, 033601 (2006).

[50] T. Chanelière, D. N. Matsukevich, S. D. Jenkins, T. A. B. Kennedy, M. S. Chapman, and A. Kuzmich, "Quantum telecommunication based on atomic cascade transitions," Phys. Rev. Lett. 96, 093604 (2006).

[51] A. Andre, M. D. Eisaman, R. L. Walsworth, A. S. Zibrov, and M. D. Lukin, "Quantum control of light using electromagnetically induced transparency," Journal of Physics $B$ 38, S589 (2005).

[52] M. D. Eisaman, L. Childress, A. Andre, F. Massou, A. S. Zibrov, and M. D. Lukin, "Shaping quantum pulses of light via coherent atomic memory," Phys. Rev. Lett. 93, 233602 (2004).

[53] D. A. Braje, V. Balic, G. Y. Yin, and S. E. Harris, "Frequency mixing using electromagnetically induced transparency in cold atoms," Phys. Rev. Lett. 93, 183601 (2004).

[54] V. Balic, D. A. Braje, P. Kolchin, G. Y. Yin, and S. E. Harris, "Generation of paired photons with controllable waveforms," Phys. Rev. Lett. 94, 183601 (2005).

[55] A. T. Black, J. K. Thompson, and V. Vuletic, "On-demand superradiant conversion of atomic spin gratings into single photons with high efficiency," Phys. Rev. Lett. 95, 133601 (2005).

[56] R. Inoue, N. Kanai, T. Yonehara, Y. Miyamoto, M. Koashi, and M. Kozuma, "Entanglement of orbital angular momentum states between an ensemble of cold atoms and a photon," quant-ph/0604165 (2006).

[57] A. P. Vandevender and P. G. Kwiat, "High efficiency single photon detection via frequency up-conversion," J. Mod. Op. 51, 1433 (2004).

[58] S. Tanzilli, W. Tittel, M. Halder, O. Alibart, P. Baldi, N. Gisin, and H. Zbinden, "A photonic quantum information interface," Nature 437, 116 (2005).

[59] S. D. Jenkins, Ph. D. thesis, 2006.

[60] S. D. Jenkins, D. N. Matsukevich, T. Chanelière, A. Kuzmich, and T. A. B. Kennedy, "Theory of dark-state polariton collapses and revivals," Phys. Rev. A 73, 021803(R) (2006).

[61] E. Knill, R. Laflamme, and G. J. Milburn, "A scheme for efficient quantum computation with linear optics.," Nature 409, 46 (2001). 
[62] M. G. Raymer and I. A. Walmsley, in Progress in Optics, edited by E. Wolf. volume XXVIII pp. 181-270 North-Holland, Amsterdam, 1996.

[63] A. Kuzmich and T. A. B. Kennedy, "Nonsymmetric entanglement of atomic ensembles," Phys. Rev. Lett. 92, 030407 (2004).

[64] M. O. Scully and M. S. Zubairy, Quantum Optics (Cambridge University Press, 1997).

[65] P. Grangier, G. Roger, and A. Aspect, "Experimental evidence for a photon anticorrelation effect on a beam splitter: A new light on single-photon interferences.," Europhys. Lett. 1, 173 (1986).

[66] A. B. U'Ren, C. Silberhorn, J. L. Ball, K. Banaszek, and I. A. Walmsley, "Characterization of the non-classical nature of conditionally prepared single photons," Phys. Rev. A 72, R021802 (2005).

[67] J. F. Clauser, M. A. Horne, A. Shimony, and R. A. Holt, "Experimental distinction between the quantum and classical field-theoretic predictions for the photoelectric effect," Phys. Rev. D 9, 853 (1974).

[68] A. Mair, J. Hager, D. F. Phillips, R. L. Walsworth, and M. D. Lukin, "Phase coherence and control of stored photonic information," Phys. Rev. A 65, 031802 (2002).

[69] J. Schmiedmayer, C. R. Ekstrom, M. S. Chapman, T. D. Hammond, and D. E. Pritchard, "Magnetic coherences in atom interferometry," Journal De Physique 4, 2029 (1994).

[70] G. A. Smith, S. Chaudhury, A. Silberfarb, I. H. Deutsch, and P. S. Jessen, "Continuous weak measurement and nonlinear dynamics in a cold spin ensemble," Phys. Rev. Lett. 93, 163602 (2004).

[71] C. Weisbuch, M. Nishioka, A. Ishikawa, and Y. Arakawa, "Observation of the coupled exciton-photon mode splitting in a semiconductor quantum microcavity," Phys. Rev. Lett. 69, 3314 (1992).

[72] J. Jacobson, S. Pau, H. Cao, G. Bjrk, and Y. Yamamoto, "Observation of excitonpolariton oscillating emission in a single-quantum-well semiconductor microcavity," Phys. Rev. A 51, 2542 (1995).

[73] M. Fleischhauer and M. D. Lukin, "Quantum memory for photons: Dark-state polaritons," Phys. Rev. A 65, 022314 (2002).

[74] D. Felinto, C. W. Chou, H. de Riedmatten, S. V. Polyakov, and H. J. Kimble, "Control of decoherence in the generation of photon pairs from atomic ensembles," Phys. Rev. A 72, 053809 (2005).

[75] D. F. Walls and G. J. Milburn, Quantum Optics (Springer-Verlag, 1994).

[76] D. N. Matsukevich, T. Chanelière, S. D. Jenkins, S.-Y. Lan, T. Kennedy, and A. Kuzmich, "Deterministic single photons via conditional quantum evolution," quant-ph/0605098, Phys. Rev. Lett. (in press) (2006). 
[77] P. Michler, A. Kiraz, C. Becher, W. V. Schoenfeld, P. M. Petroff, L. D. Zhang, E. Hu, and A. Imamoglu, "A quantum dot single-photon turnstile device," Science 290, 2282 (2000).

[78] C. Santori, M. Pelton, G. Solomon, Y. Dale, and Y. Yamamoto, "Triggered single photons from a quantum dot," Phys. Rev. Lett. 86, 1502 (2001).

[79] M. Pelton, C. Santori, J. Vuckovic, B. Zhang, G. S. Solomon, J. Plant, and Y. Yamamoto, "Efficient source of single photons: A single quantum dot in a micropost microcavity," Phys. Rev. Lett. 89, 233602 (2002).

[80] R. Brouri, A. Beveratos, J. P. Poizat, and P. Grangier, "Photon antibunching in the fluorescence of individual color centers in diamond," Optics Letters 25, 1294 (2000).

[81] C. Kurtsiefer, S. Mayer, P. Zarda, and H. Weinfurter, "Stable solid-state source of single photons," Phys. Rev. Lett. 85, 290 (2000).

[82] B. Darquie, M. P. A. Jones, J. J. Dingjan, J. Beugnon, S. Bergamini, Y. Sortais, G. Messin, A. Browaeys, and P. Grangier, "Controlled single-photon emission from a single trapped two-level atom," Science 309, 454 (2005).

[83] M. Keller, B. Lange, K. Hayasaka, W. Lange, and H. Walther, "Continuous generation of single photons with controlled waveform in an ion-trap cavity system," Nature $\mathbf{4 3 1}$, 1075 (2004).

[84] R. Lounis and W. E. Moerner, "Single photons on demand from a single molecule at room temperature," Nature 407, 491 (2000).

[85] C. K. Hong and L. Mandel, "Experimental realization of a localized one-photon state," Phys. Rev. Lett. 56, 58 (1986).

[86] M. D. Eisaman, A. Andre, F. Massou, M. Fleischhauer, A. S. Zibrov, and M. D. Lukin, "Electromagnetically induced transparency with tunable single-photon pulses," $\mathrm{Na}$ ture 438, 837 (2005).

[87] W. P. Smith, J. E. Reiner, L. A. Orozco, S. Kuhr, and H. M. Wiseman, "Capture and release of a conditional state of a cavity QED system by quantum feedback," Phys. Rev. Lett. 89, 133601 (2002).

[88] T. Fischer, P. Maunz, P. W. H. Pinkse, T. Puppe, and G. Rempe, "Feedback on the motion of a single atom in an optical cavity," Phys. Rev. Lett. 88, 163002 (2002).

[89] C. W. Chou, H. de Riedmatten, D. Felinto, S. V. Polyakov, S. J. van Enk, and H. J. Kimble, "Measurement-induced entanglement for excitation stored in remote atomic ensembles," Nature 438, 828 (2005).

[90] R. Loudon, The Quantum Theory of Light (Oxford Press, 2000).

[91] L.-M. Duan, J. I. Cirac, and P. Zoller, "Three-dimensional theory for interaction between atomic ensembles and free-space light," Phys. Rev. A 66, 023818 (2002).

[92] M. Horodecki, P. Horodecki, and R. Horodecki, "General teleportation channel, singlet fraction, and quasidistillation," Phys. Rev. A 60, 1888 (1999). 
[93] C. H. Bennett, D. P. DiVincenzo, J. A. Smolin, and W. K. Wootters, "Mixed-state entanglement and quantum error correction," Phys. Rev. A 54, 3824 (1996).

[94] Y. L. Lim, A. Beige, and L. C. Kwek, "Repeat-until-success linear optics distributed quantum computing," Phys. Rev. Lett. 95, 030505 (2005).

[95] S. D. Barrett and P. Kok, "Efficient high-fidelity quantum computation using matter qubits and linear optics," Phys. Rev. A 71, 060310 (2005).

[96] L. Mandel, "Quantum effects in one-photon and two-photon interference," Rev. Mod. Phys. 71, S274 (1999).

[97] A. Zeilinger, "Experiment and the foundations of quantum physics," Rev. Mod. Phys. 71, S288 (1999).

[98] J. F. Clauser, M. A. Horne, A. Shimony, and R. A. Holt, "Proposed experiment to test local hidden-variable theories," Phys. Rev. Lett. 23, 880 (1969).

[99] C. Chou, S. Polyakov, A. Kuzmich, and H. Kimble, "Single-photon generation from stored excitation in an atomic ensemble," Phys. Rev. Lett. 92, 213601 (2004).

[100] S. V. Polyakov, C. W. Chou, D. Felinto, and H. J. Kimble, "Temporal dynamics of photon pairs generated by an atomic ensemble," Phys. Rev. Lett. 93, 263601 (2004).

[101] D. N. Matsukevich, T. Chanelière, S. D. Jenkins, S.-Y. Lan, T. Kennedy, and A. Kuzmich, "Entanglement of remote atomic qubits," Phys. Rev. Lett. 96, 030405 (2006).

[102] M. Aspelmeyer, H. R. Bohm, T. Gyatso, T. Jennewein, R. Kaltenbaek, M. Lindenthal, G. Molina-Terriza, A. Poppe, K. Resch, M. Taraba, R. Ursin, P. Walther, and A. Zeilinger, "Long-distance free-space distribution of quantum entanglement," Science 301, 621 (2003).

[103] C. A. Sackett, D. Kielpinski, B. E. King, C. Langer, V. Meyer, C. J. Myatt, M. Rowe, Q. A. Turchette, W. M. Itano, D. J. Wineland, and I. C. Monroe, "Experimental entanglement of four particles," Nature 404, 256 (2000).

[104] C. Cabrillo, J. I. Cirac, P. G. Fernandez, and P. Zoller, "Quantum repeaters: The role of imperfect local operations in quantum communication," Phys. Rev. A 59, 1025 (1999).

[105] S. Bose, P. Knight, M. B. Plenio, and V. Vedral, "Proposal for teleportation of an atomic state via cavity decay," Phys. Rev. Lett. 83, 5158 (1999).

[106] A. S. Sorensen and K. Molmer, "Probabilistic generation of entanglement in optical cavities," Phys. Rev. Lett. 90, 127903 (2003).

[107] L.-M. Duan, "Entangling many atomic ensembles through laser manipulation," Phys. Rev. Lett. 88, 170402 (2002).

[108] D. Tupa and L. W. Anderson, "Effect of radiation trapping on the polarization of an optically pumped alkali-metal vapor in a weak magnetic-field," Phys. Rev. A 36, 2142 (1987). 
[109] S. J. van Enk and H. J. Kimble, "Comment on "Quantum state transfer between matter and light"," Science 309, 1187b (2005).

[110] D. N. Matsukevich and A. Kuzmich, "Response to Comment on "Quantum state transfer between matter and light","Science 309, 1187c (2005).

[111] Q. A. Turchette, C. S. Wood, B. E. King, C. J. Myatt, D. Leibfried, W. M. Itano, C. Monroe, and D. J. Wineland, "Deterministic entanglement of two trapped ions," Phys. Rev. Lett. 81, 3631 (1998).

[112] M. Saffman and T. G. Walker, "Entangling single- and N-atom qubits for fast quantum state detection and transmission," Phys. Rev. A 72, 042302 (2005).

[113] D. D. Yavuz, P. B. Kulatunga, E. Urban, T. A. Johnson, N. Proite, T. Henage, T. G. Walkerand, and M. Saffman, "Fast ground state manipulation of neutral atoms in microscopic optical traps," Phys. Rev. Lett 96, 063001 (2006).

[114] M. Arndt, S. I. Kanorsky, A. Weis, and T. W. Hansch, "Long electronic spin relaxation times of Cs atoms in solid ${ }^{4}$ He," Phys. Rev. Lett. 74, 1359 (1995).

[115] N. E. Rehler and J. H. Eberly, "Superradiance," Phys. Rev. A 3, 1735 (1971).

[116] R. Friedberg and S. R. Hartmann, "Superradiant lifetime: Its definitions and relation to absorption length," Phys. Rev. A 13, 495 (1976).

[117] F. Vrehen, H. M. J. Hikspoors, and H. M. Gibbs, "Single-pulse superfluorescence in Cesium," Phys. Rev. Lett. 39, 547 (1977).

[118] J. D. Franson, "Bell inequality for position and time," Phys. Rev. Lett. 62, 2205 (1989).

[119] D. Branning, W. P. Grice, R. Erdmann, and I. A. Walmsley, "Engineering the indistinguishability and entanglement of two photons," Phys. Rev. Lett. 83, 955 (1999).

[120] C. K. Law, I. A. Walmsley, and J. H. Eberly, "Continuous frequency entanglement: effective finite hilbert space and entropy control," Phys. Rev. Lett. 84, 5304 (2000).

[121] A. Aspect, P. Grangier, and G. Roger, "Experimental tests of realistic local theories via Bell's theorem," Phys. Rev. Lett. 47, 460 (1981). 